\title{
MONOMORPHISMS IN CATEGORIES OF LOG SCHEMES
}

\author{
SHINICHI MochIZUKI
}

\begin{abstract}
In the present paper, we study category-theoretic properties of monomorphisms in categories of $\log$ schemes. This study allows one to give a purely category-theoretic reconstruction of the $\log$ scheme that gave rise to the category under consideration. We also obtain analogous results for categories of schemes of locally finite type over the ring of rational integers that are equipped with "archimedean structures". Such reconstructions were discussed in two previous papers by the author, but these reconstructions contained some errors, which were pointed out to the author by C. Nakayama and Y. Hoshi. These errors revolve around certain elementary combinatorial aspects of fan decompositions of two-dimensional rational polyhedral cones-i.e., of the sort that occur in the classical theory of toric varieties - and may be repaired by applying the theory developed in the present paper.
\end{abstract}

\section{CONTENTS}

§0. Notations and conventions 369

§1. Generalities on monomorphisms and minimal points 373

§2. The scheme structure of submonic log schemes 387

§3. Seamless partitions of orientable log schemes 398

$\S 4$. Category-theoretic representation of archimedean structures 414

$\begin{array}{ll}\text { Appendix } & 426\end{array}$

\section{Introduction}

The purpose of the present paper is to study, in some detail, various aspects of the structure of categories of log schemes that revolve around the behavior of monomorphisms in such categories. This study leads naturally to a purely category-theoretic reconstruction of the log scheme that gave rise to the category under consideration. Our main result is the following [cf. Theorem 3.8, (iii)].

2010 Mathematics Subject Classification. Primary 14A15; Secondary 14G40.

Key words and phrases. Log scheme, scheme, category, anabelian geometry, archimedean structure.

Received April 30, 2014; revised November 25, 2014. 
Theorem A (Category-theoretic reconstruction of $\log$ schemes). For $i=1,2$, let $X_{i}^{\log }$ be a locally noetherian fs log scheme [cf. the discussion entitled "Log schemes" in §0]. For $i=1,2$, we shall write $\operatorname{Sch}^{\log }\left(X_{i}^{\log }\right)$ for the category of noetherian $f_{s} \log$ schemes of finite type over $X_{i}^{\log }$ and morphisms of finite type [cf. the discussion at the beginning of $\$ 1$ for more details]. Let

$$
\Phi: \operatorname{Sch}^{\log }\left(X_{1}^{\log }\right) \stackrel{\sim}{\rightarrow} \operatorname{Sch}^{\log }\left(X_{2}^{\log }\right)
$$

be an [arbitrary!] equivalence of categories. Then there exists a unique isomorphism of log schemes

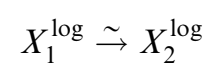

such that $\Phi$ is isomorphic to the equivalence of categories induced by this isomorphism of log schemes $X_{1}^{\log } \stackrel{\sim}{\rightarrow} X_{2}^{\log }$. schemes

We also obtain analogous results for categories of locally noetherian $f_{s} \log$

$$
\text { "SCH }{ }^{\log }(-) "
$$

[cf. Theorem 4.6, (iv)], as well as for versions

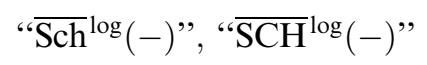

of the categories " $\mathrm{Sch}^{\log }(-)$ ", "SCH${ }^{\log }(-)$ " for schemes of locally finite type over $\mathbf{Z}$ that are equipped with "archimedean structures" [cf. Theorem 4.8, (iv)].

The theory exposed in the present paper arose as an attempt to correct errors, pointed out to the author by Chikara Nakayama and Yuichiro Hoshi in June 2013, in the theory of [4], \$2. These errors concern the category-theoretic properties of monomorphisms in categories of log schemes and are discussed in more detail in Example 0.3 and Remark 1.4.1 of the present paper.

At the level of main results of the paper [4], these errors in the theory of [4], §2, do not affect the proof of [4], Theorem A, given in [4], §1, but they do affect the proof - although not the validity! —of [4], Theorem B. This result [4], Theorem $\mathrm{B}$, is given a correct proof in $\$ 3$ of the present paper and corresponds precisely to Theorem A [stated above].

At the level of main results of papers of the author subsequent to [4], the only place where the errors in the theory of [4], §2, have an effect is in the portion of the proof of the main result of [5] [i.e., [5], Theorem 5.1] that involves the theory of [5], $\$ 4$. The affected portions of [5], $\$ 4$, are discussed in more detail in the introduction to $\S 4$ of the present paper. The main result [5], Theorem 5.1, of [5] is given a correct proof in $\S 4$ of the present paper and corresponds precisely to Theorem 4.8, (iv) [quoted above].

At the level of individual propositions, lemmas, corollaries, theorems, and examples [i.e., which do not necessarily qualify as "main results" of the paper under consideration], a detailed discussion of the affected portions of [4] and [5] may be found in the Appendix to the present paper. 
One important invariant of the structure of an fs log scheme is the rank of the groupification of the fiber of the characteristic sheaf associated to the log structure at a geometric point of the underlying scheme of the log scheme [cf. Definition 1.2, (i)]. For instance, when this rank is equal to 0 at all geometric points, the $\log$ structure of the fs $\log$ scheme under consideration is trivial. One central theme of the theory of the present paper consists of the phenomenon that

the theory of category-theoretic properties of monomorphisms exhibits quite substantive qualitative differences, depending upon whether or not it holds that the ranks just referred to are $\leq 1$.

When it holds that these rank are $\leq 1$, the fs log scheme under consideration will be referred to in the present paper as submonic [cf. Definition 1.2, (i)].

Thus, in some sense, the simplest "borderline case" between submonic and non-submonic fs $\log$ schemes is the case of a log scheme whose underlying scheme is the spectrum of a field whose absolute Galois group acts trivially on geometric fibers of the characteristic sheaf associated to the log structure, and for which the rank of the groupification of each such geometric fiber of the characteristic sheaf is equal to 2. In this case, the log scheme under consideration will be referred to as log-nodal [cf. Definition 1.2, (i)].

One important feature of the category-theoretic properties of monomorphisms in categories of $\log$ schemes lies in the observation that

these category-theoretic properties of monomorphisms take on a particularly straightforward and intuitive form whenever it holds that the various fs $\log$ schemes under consideration are all submonic.

This observation is one of the main themes of the theory discussed in $\S 1$ of the present paper. Roughly speaking, the errors pointed out by Nakayama and Hoshi in the theory of [4], §2, may be summarized as follows:

the author wrote [4], §2, under the misunderstanding that this "straightforward" and "intuitive" approach to category-theoretic properties of monomorphisms holds even if the various fs log schemes under consideration are not necessarily submonic.

On the other hand, it turns out [cf. the theory of $\$ 2$ of the present paper] that the various complications that occur in the study of the category-theoretic properties of monomorphisms of arbitrary non-submonic fs log schemes already appear in the case of log-nodal fs $\log$ schemes. Moreover, it turns out that

these complications essentially revolve around various combinatorial aspects of fan decompositions of two-dimensional rational polyhedral cones, i.e., of the sort that occur in the classical theory of toric varieties. 
These elementary combinatorial aspects are reviewed in $\S 0$ of the present paper.

The theory developed in the present paper may be summarized as follows. In $\S 1$, we introduce basic terminology and discuss various generalities concerning monomorphisms in categories of log schemes. In particular, we discuss [cf., especially, Lemma 1.5] how the elementary combinatorics of two-dimensional fan decompositions reviewed in $\S 0$ may be interpreted in the context of categories of $\log$ schemes. In $\S 2$, we apply these elementary combinatorics of two-dimensional fan decompositions [cf. Proposition 2.3] to show, in effect, that certain connectedness properties of such fan decompositions allow one to give a category-theoretic characterization of submonic $f_{s}$ log schemes. We then proceed to give, in Theorem 2.6, a category-theoretic reconstruction of the scheme structure of a submonic $f_{s} \log$ scheme. This reconstruction is quite "straightforward" and "intuitive" and amounts, in essence, to an application of the techniques of [4], §2. In the remainder of $\S 2$, we show [cf. Corollary 2.12] that the various complications that arise in the case of arbitrary non-submonic fs log schemes amount, in essence, to the issue of giving a category-theoretic algorithm that allows one

to distinguish a log-nodal fs log scheme from a nontrivial log étale localization of such a log-nodal fs $\log$ scheme [i.e., of the sort that arises from a nontrivial two-dimensional fan decomposition].

Such a category-theoretic algorithm is furnished, in effect, by the theory of seamless partitions of orientable log schemes developed in $\$ 3$ [cf. Theorem 3.6]. This theory may be regarded as a translation into category theory of the elementary observation that

a nontrivial two-dimensional fan decomposition may be distinguished from a trivial two-dimensional fan decomposition by considering the "seamless partition" constituted by the various constituent cones of the fan decomposition.

Finally, in $\S 4$, we observe that the theory developed in $\S 1, \S 2, \S 3$ may be generalized, without any essential complications, to the case of fs log schemes of locally finite type over $\mathbf{Z}$ that are equipped with archimedean structures [cf. Theorems 4.3, 4.8]. Such generalizations allow one to avoid the difficulties that arise from applying the erroneous portions of [4], $\$ 2$, in the theory of [5], §4, i.e., by, in essence, isolating the [easily resolved] submonic aspects of these difficulties from the [more subtle!] non-submonic aspects of these difficulties.

Acknowledgements. This paper owes its existence to the discovery by Chikara Nakayama and Yuichiro Hoshi of various errors [cf. Example 0.3; Remark 1.4.1] in the arguments of [4], §2. The author wishes to express his gratitude to Nakayama and Hoshi for their careful reading of [4]. 


\section{Section 0: Notations and conventions}

\section{Numbers:}

We will denote by $\mathbf{N}$ the set of natural numbers, by which we mean the set of integers $n \geq 0$, and by $\mathbf{Z}$ the ring of rational integers. By a slight abuse of notation, we shall also use the notation $\mathbf{N}, \mathbf{Z}$ to denote the corresponding monoids. We shall denote by $\mathbf{Q}_{\geq 0}$ the additive monoid of nonnegative rational numbers.

\section{Generalities on monoids:}

We shall refer to a finitely generated, saturated [cf. [2], §1.1] monoid that has no nonzero invertible elements as an $f s$ monoid. Thus, if $P$ is an fs monoid, then the natural homomorphism of monoids $P \rightarrow P^{\text {gp }}$ from $P$ to its groupification $P^{\mathrm{gp}}$ is injective, and $P^{\mathrm{gp}}$ is a finitely generated free abelian group. We shall refer to the rank of $P^{\mathrm{gp}}$ as the $\operatorname{rank} \operatorname{rk}(P)$ of the $f s$ monoid $P$.

A homomorphism of monoids $\phi: P \rightarrow Q$ between monoids $P, Q$ will be called positive if $\phi$ maps every nonzero element of $P$ to a nonzero element of $Q$. A nonzero element $a \in P$ of a monoid $P$ will be called a sum-dominator if there exists a positive integer $n$ such that $n \cdot a$ may be written as the sum of a finite collection of generators of $P$. Thus, if $\phi: P \rightarrow Q$ is a nonzero homomorphism [i.e., a homomorphism that maps any collection of generators of $P$ to a subset of $Q$ that contains at least one nonzero element!] from an arbitrary monoid $P$ to an $f s$ monoid $Q$, and $a \in P$ is a sum-dominator, then $\phi(a) \neq 0$. We shall say that a homomorphism of monoids $\phi: P \rightarrow Q$ is sum-dominating if it maps every nonzero element of $P$ to a sum-dominator of $Q$. Thus, a sumdominating homomorphism is necessarily positive.

Let $P$ be an $f_{s}$ monoid. Thus, in the terminology of the discussion entitled "Monoids" of [6], $§ 0, P$ is sharp, integral, and saturated. In particular, it makes sense to speak of the perfection $P^{\mathrm{pf}}$ of $P$, as well as of the set of primes $\operatorname{Prime}(P)$ of $P$-cf. the discussion entitled "Monoids" of [6], §0, for more details.

\section{Rank two fs monoids:}

Now let us suppose that $P$ is an $f_{s}$ monoid of rank two. Then we recall that there exists an isomorphism of monoids

$$
P^{\mathrm{pf}} \stackrel{\sim}{\rightarrow} \mathbf{Q}_{\geq 0} \oplus \mathbf{Q}_{\geq 0}
$$

[cf. [3], Proposition 1.7]. In particular, one verifies immediately that the set of primes $\operatorname{Prime}(P)=\operatorname{Prime}\left(P^{\mathrm{pf}}\right)$ is of cardinality two. Write $\operatorname{Prime}(P)=\operatorname{Prime}\left(P^{\mathrm{pf}}\right)$ $=\left\{\mathfrak{p}_{1}, \mathfrak{p}_{2}\right\}$. Thus, for each $i=1,2, \mathfrak{p}_{i}$ may be regarded as a collection of elements of $P^{\mathrm{pf}}$, which generates a submonoid $P_{\mathfrak{p}_{i}}^{\mathrm{pf}} \subseteq P^{\mathrm{pf}}$. For simplicity, let us write $P_{i} \stackrel{\text { def }}{=} P_{\mathfrak{p}_{i}}^{\mathrm{pf}}$. Then one verifies immediately that the two direct summands of the codomain of the isomorphism of the above display correspond precisely to $P_{1}, P_{2}$, i.e., we have a natural isomorphism

$$
P_{1} \oplus P_{2} \stackrel{\sim}{\rightarrow} P^{\mathrm{pf}}
$$


and noncanonical isomorphisms of abstract monoids $P_{1} \cong P_{2} \cong \mathbf{Q}_{\geq 0}$. In particular, these two direct summands are preserved, up to possible permutation, by any automorphism of the monoid $P^{\mathrm{pf}}$. Note that [since the monoid $\mathbf{Q}_{\geq 0}$ has no nontrivial automorphisms of finite order] these observations imply that

any finite subgroup of $\operatorname{Aut}\left(P^{\mathrm{pf}}\right)$ - or, indeed, of $\operatorname{Aut}(P)\left(\hookrightarrow \operatorname{Aut}\left(P^{\mathrm{pf}}\right)\right)-$ is of order $\leq 2$.

Next, let

$$
\phi_{0}: P \rightarrow J_{0} \stackrel{\text { def }}{=} \mathbf{N}
$$

be a positive homomorphism that induces a surjection on groupifications $\phi_{0}^{\mathrm{gp}}$ : $P^{\mathrm{gp}} \rightarrow J_{0}^{\mathrm{gp}}=\mathbf{Z}$. Thus, $\operatorname{Ker}\left(\phi_{0}^{\mathrm{gp}}\right) \cong \mathbf{Z}$. Fix a nonzero element $a \in \operatorname{Ker}\left(\phi_{0}^{\mathrm{gp}}\right) \subseteq$ $P^{\mathrm{gp}}$. For $i=1,2$, write

$$
(P \subseteq) J_{i} \subseteq P^{\mathrm{gp}}
$$

for the saturation [cf. [4], Lemma 2.5, (ii)] of the submonoid of $P^{\text {gp }}$ generated by $P$ and $a$ if $i=1$ (respectively, $-a$ if $i=2$ ) and

$$
\phi_{i}: P \hookrightarrow J_{i}
$$

for the natural inclusion. Thus, $P^{\mathrm{gp}}=J_{i}^{\mathrm{gp}}$ for $i=1,2$. One verifies immediately that, up to a possible permutation of the indices " 1 " and " 2 ", the submonoids $J_{1}$ and $J_{2}$ of $P^{\mathrm{gp}}$ are independent of the choice of $a$. Moreover, we observe that it follows immediately from the definition of $J_{1}$ and $J_{2}$ that

if $i=0$ (respectively, $i=1, \quad i=2$ ), then a positive homomorphism $\phi: P \rightarrow \mathbf{N}$ factors, via $\phi_{i}: P \rightarrow J_{i}$, through a positive homomorphism $J_{i} \rightarrow \mathbf{N}$ if and only if the homomorphism induced on groupifications $\phi^{\mathrm{gp}}: P^{\mathrm{gp}} \rightarrow \mathbf{Z}$ satisfies the condition $\phi^{\mathrm{gp}}(a)=0$ (respectively, $\phi^{\mathrm{gp}}(a)>0$; $\left.\phi^{\mathrm{gp}}(a)<0\right)$.

In this situation, we shall refer to $J_{1}$ and $J_{2}$ as bisecting monoids of $P$ at $\phi_{0}$. Before proceeding, we observe the following "continuity property" of bisecting monoids:

Suppose that $P^{*} \subseteq P^{\text {gp }}$ is a rank two $f$ s monoid that arises as a submonoid of $P^{\text {gp }}$ that contains $P$. For $i=1,2$, suppose that there exists a homomorphism $\psi_{i}: P^{*} \rightarrow \mathbf{N}$ whose restriction to $P$ factors, via $\phi_{i}: P \rightarrow J_{i}$, through a positive homomorphism $J_{i} \rightarrow \mathbf{N}$. Then $\phi_{0}: P \rightarrow \mathbf{N}$ extends to a positive homomorphism $\psi_{0}: P^{*} \rightarrow \mathbf{N}$.

Indeed, if $\phi_{0}$ does not admit such an extension $\psi_{0}$, then it follows that there exist nonzero elements $b \in P, c \in P^{*}$ such that $a+b+c=0$ for some element $a \in \operatorname{Ker}\left(\phi_{0}^{\mathrm{gp}}\right) \subseteq P^{\mathrm{gp}}$. Then it follows from the above discussion of bisecting monoids that, for some $i \in\{1,2\}, \psi_{i}^{\mathrm{gp}}(a) \geq 0$. Since the restriction of $\psi_{i}$ to $P$ is 
a positive homomorphism, we thus conclude that $0=\psi_{i}^{\mathrm{gp}}(a)+\psi_{i}^{\mathrm{gp}}(b)+\psi_{i}^{\mathrm{gp}}(c)>$ $0 \in \mathbf{N}$, a contradiction. This completes the proof of this "continuity property".

Bisecting monoids may be understood more explicitly if one passes to perfections. Indeed, by restricting our attention to perfections, one verifies immediately that we may assume without loss of generality that

$$
P^{\mathrm{pf}}=\mathbf{Q}_{\geq 0} \oplus \mathbf{Q}_{\geq 0}, \quad P_{1}=\mathbf{Q}_{\geq 0} \oplus 0, \quad P_{2}=0 \oplus \mathbf{Q}_{\geq 0},
$$

and that $\phi_{0}^{\mathrm{pf}}: P^{\mathrm{pf}} \rightarrow \mathbf{Q}_{\geq 0}$ is the homomorphism determined by sending $(1,0)$ and $(0,1)$ to 1 . Then one computes easily that, if one takes $a \stackrel{\text { def }}{=}(1,-1)$, then $J_{1}^{\text {pf }}$ is equal to the perfection of the submonoid of $\left(P^{\mathrm{pf}}\right)^{\mathrm{gp}}=\mathbf{Q} \oplus \mathbf{Q}$ generated by $(0,1)$ and $(1,-1)$, while $J_{2}^{\mathrm{pf}}$ is equal to the perfection of the submonoid of $\left(P^{\mathrm{pf}}\right)^{\mathrm{gp}}=\mathbf{Q} \oplus \mathbf{Q}$ generated by $(1,0)$ and $(-1,1)$. Thus, if $\phi^{\mathrm{pf}}$ maps

$$
(1,0) \mapsto \alpha ; \quad(0,1) \mapsto \beta
$$

for $\alpha, \beta \in \mathbf{Q}_{\geq 0}$, then one verifies immediately that $\phi^{\mathrm{pf}}: P^{\mathrm{pf}} \rightarrow \mathbf{Q}_{\geq 0}$ factors, via $\phi_{i}^{\mathrm{pf}}: P^{\mathrm{pf}} \rightarrow J_{i}^{\mathrm{pf}}$, through a positive homomorphism $J_{i}^{\mathrm{pf}} \rightarrow \mathbf{Q}_{\geq 0}$

for $i=0$ (respectively, $i=1 ; i=2) \Leftrightarrow \alpha=\beta$ (respectively, $\alpha>\beta ; \alpha<\beta$ ).

In the present paper, we shall often consider certain sequences of submonoids satisfying certain special properties, as in the following examples.

Example 0.1 (Submonoids converging from one side). Let $P$ be an $f_{S}$ monoid of rank two, ${ }^{\infty} P \subseteq P^{\mathrm{gp}}$ a bisecting monoid of $P$ at some positive homomorphism ${ }^{\infty} \phi: P \rightarrow \mathbf{N}$. Then there exists an infinite descending sequence

$$
P \subseteq{ }^{\infty} P \subseteq \cdots \subseteq{ }^{n} P \subseteq \cdots \subseteq{ }^{1} P \subseteq{ }^{0} P
$$

-where $n \in \mathbf{N}$-of submonoids of $P^{\mathrm{gp}}$ such that every positive homomorphism $\phi:{ }^{\infty} P \rightarrow \mathbf{N}$ factors through a positive homomorphism ${ }^{n} P \rightarrow \mathbf{N}$ for some $n$ [which may depend on $\phi]$, and, moreover, for each $m \in \mathbf{N},{ }^{m} P$ is a bisecting monoid of $P$ [hence, in particular, an fs monoid of rank two] whose image ${ }^{\infty} \phi^{\mathrm{gp}}\left({ }^{m} P\right)$ via ${ }^{\infty} \phi^{\mathrm{gp}}: P^{\mathrm{gp}} \rightarrow \mathbf{Z}$ contains both positive and negative elements. Indeed, by reasoning as in the above discussion, one reduces immediately to the verificationsay, in the case where $P^{\mathrm{pf}}=\mathbf{Q}_{\geq 0} \oplus \mathbf{Q}_{\geq 0},{ }^{\infty} \phi^{\mathrm{pf}}$ is the homomorphism $P^{\mathrm{pf}}=$ $\mathbf{Q}_{\geq 0} \oplus \mathbf{Q}_{\geq 0} \rightarrow \mathbf{Q}_{\geq 0}$ given by $(\alpha, \beta) \mapsto \alpha+\beta$, and ${ }^{\infty} P^{\mathrm{pf}}$ is the perfection of the submonoid of $\mathbf{Q} \oplus \mathbf{Q}$ generated by $(-1,1)$ and $(1,0)$ - of the existence of an infinite descending sequence

$$
P^{\mathrm{pf}} \subseteq{ }^{\infty} P^{\mathrm{pf}} \subseteq \cdots \subseteq{ }^{n} P^{\mathrm{pf}} \subseteq \cdots \subseteq{ }^{1} P^{\mathrm{pf}} \subseteq{ }^{0} P^{\mathrm{pf}}
$$

-where $n \in \mathbf{N}$-of submonoids of $\left(P^{\mathrm{pf}}\right)^{\mathrm{gp}}$ such that every positive homomorphism $\psi:{ }^{\infty} P^{\mathrm{pf}} \rightarrow \mathbf{Q}_{\geq 0}$ factors through a positive homomorphism ${ }^{n} P^{\mathrm{pf}} \rightarrow \mathbf{Q}_{\geq 0}$ for some $n$ [which may depend on $\psi$ ], and, moreover, for each $m \in \mathbf{N},{ }^{m} P^{\text {pf }}$ is the perfection of a finitely generated submonoid of $\mathbf{Q} \oplus \mathbf{Q}$ such that ${ }^{m} P \stackrel{m}{=} \stackrel{\text { def }}{=} P^{\text {pf }} \cap$ $P^{\mathrm{gp}}$ [so ${ }^{m} P^{\mathrm{pf}}$ may be identified with the perfection of ${ }^{m} P$, as the notation 
suggests!] is a bisecting monoid of $P$ whose image ${ }^{\infty} \phi^{\mathrm{gp}}\left({ }^{m} P\right)$ contains both positive and negative elements. Such an infinite descending sequence may be obtained, for instance, by taking ${ }^{n} P^{\mathrm{pf}}$ to be the perfection of the submonoid of $\mathbf{Q} \oplus \mathbf{Q}$ generated by $\left(-1,1-\frac{1}{n+2}\right)$ and $(1,0)$.

Example 0.2 (Submonoids converging from the center). Let $P$ be an $f_{s}$ monoid of rank two. Then there exists an infinite descending sequence

$$
P \subseteq \cdots \subseteq{ }^{n} P \subseteq \cdots \subseteq{ }^{1} P \subseteq{ }^{0} P
$$

- where $n \in \mathbf{N}$-of submonoids of $P^{\mathrm{gp}}$ such that every positive homomorphism $\phi: P \rightarrow \mathbf{N}$ factors through a positive homomorphism ${ }^{n} P \rightarrow \mathbf{N}$ for some $n$ [which may depend on $\phi$ ], and, moreover, for each $m \in \mathbf{N}$, the inclusion $P \hookrightarrow{ }^{m} P$ is a sum-dominating homomorphism of $f_{s}$ monoids. Indeed, by reasoning as in the above discussion, one reduces immediately to the verification, in the case where $P^{\mathrm{pf}}=\mathbf{Q}_{\geq 0} \oplus \mathbf{Q}_{\geq 0}$, of the existence of an infinite descending sequence

$$
P^{\mathrm{pf}} \subseteq \cdots \subseteq{ }^{n} P^{\mathrm{pf}} \subseteq \cdots \subseteq{ }^{1} P^{\mathrm{pf}} \subseteq{ }^{0} P^{\mathrm{pf}}
$$

- where $n \in \mathbf{N}$-of perfections of finitely generated submonoids of $\left(P^{\mathrm{pf}}\right)^{\mathrm{gp}}$ such that every positive homomorphism $\psi: P^{\mathrm{pf}} \rightarrow \mathbf{Q}_{\geq 0}$ factors through a positive homomorphism ${ }^{n} P^{\mathrm{pf}} \rightarrow \mathbf{Q}_{\geq 0}$ for some $n$ [which may depend on $\psi$ ], and, moreover, for each $m \in \mathbf{N}$, the inclusion $P \stackrel{m}{\hookrightarrow} P \stackrel{\text { def }}{=} m P^{\text {pf }} \cap P^{\mathrm{gp}}$ [so ${ }^{m} P^{\mathrm{pf}}$ may be identified with the perfection of ${ }^{m} P$, as the notation suggests!] induced by the inclusion $P^{\mathrm{pf}} \hookrightarrow{ }^{m} P^{\mathrm{pf}}$ is a sum-dominating homomorphism of $f s$ monoids. Such an infinite descending sequence may be obtained, for instance, by taking ${ }^{n} P^{\mathrm{pf}}$ to be the perfection of the submonoid of $\mathbf{Q} \oplus \mathbf{Q}$ generated by $\left(1,-\frac{1}{n+2}\right)$ and $\left(-\frac{1}{n+2}, 1\right)$. Finally, we observe that this explicit construction shows that the ${ }^{n} P$ may be chosen so as to be preserved by any finite group of automorphisms of $P$.

\section{Log schemes:}

If $X$ is a scheme, then we shall write

$$
X_{\text {red }} \subseteq X
$$

for the closed subscheme determined by equipping the underlying topological space of the scheme $X$ with the reduced induced scheme structure. If $X$ is the underlying scheme of a log scheme $X^{\log }$ [cf. [1], \$1.2], then we shall write $X_{\text {red }}^{\log }$ for the $\log$ scheme determined by restricting the $\log$ structure of $X^{\log }$ to $X_{\text {red }} \subseteq X$.

We shall use the terms log étale (respectively, log smooth) to refer to morphisms between log schemes which are "étale" (respectively, "smooth") in the sense of [1], §3.3 (respectively, [1], §3.3; [2], §8.1).

We use the term " $f s$ log scheme" to refer to a log scheme which is fine [cf. [1], §2.3] and saturated [cf. [the evident étale generalization of] [2], §1.5]. We 
shall refer to a $\log$ scheme as noetherian (respectively, locally noetherian) if its underlying scheme is noetherian (respectively, locally noetherian). We shall say that a morphism of log schemes is of finite type if its underlying morphism of schemes is of finite type. We shall say that a morphism of log schemes is an open immersion if its underlying morphism of schemes is an open immersion, and, moreover, the log structure on its domain is obtained as the pull-back of the log structure on its codomain. We shall say that a morphism of log schemes is dominant if its underlying morphism of schemes is dominant.

We recall from [4], Lemma 2.6, (i), (ii), (iii), that the natural morphism from the underlying scheme of any fiber product in the category of locally noetherian $f_{s} \log$ schemes to the corresponding fiber product of underlying schemes is finite. On the other hand, this natural morphism is not necessarily surjective! That is to say, the isomorphism asserted [unfortunately, without an explicit proof!] in [4], Lemma 2.6, (ii), is false. Indeed, the following example constitutes a counterexample to this isomorphism.

Example 0.3 (Empty fiber products of log schemes). Consider the fiber product determined by the diagram of log schemes

$$
X^{\log } \rightarrow Z^{\log } \leftarrow Y^{\log }
$$

obtained by equipping the diagram of schemes

$$
X \stackrel{\text { def }}{=} \operatorname{Spec}(k) \rightarrow Z \stackrel{\text { def }}{=} \operatorname{Spec}(k) \leftarrow Y \stackrel{\text { def }}{=} \operatorname{Spec}(k)
$$

- where $k$ is a field, and the arrows are the identity morphisms - with the log structures determined by the diagram of monoids

$$
P_{X} \stackrel{\text { def }}{=}\langle(1,0) ;(-1,1)\rangle \supseteq P_{Z} \stackrel{\text { def }}{=} \mathbf{N} \oplus \mathbf{N} \subseteq P_{Y} \stackrel{\text { def }}{=}\langle(1,-1) ;(0,1)\rangle
$$

-where the notation " $\langle-\rangle$ " denotes the submonoid of $P_{Z}^{\mathrm{gp}}=\mathbf{N}^{\mathrm{gp}} \oplus \mathbf{N}^{\mathrm{gp}}=$ $\mathbf{Z} \oplus \mathbf{Z}$ generated by the element(s) in brackets - and the morphisms of monoids $P_{X} \rightarrow k, P_{Y} \rightarrow k, P_{Z} \rightarrow k$ that map $0 \mapsto 1 \in k$ and all nonzero elements of the domain to $0 \in k$. Then one verifies immediately that this fiber product is, in fact, empty, despite the fact that $X \times_{Y} Z=\operatorname{Spec}(k) \neq \emptyset$.

\section{Section 1: Generalities on monomorphisms and minimal points}

In the present $\S 1$, we discuss various definitions and generalities related to monomorphisms and "minimal points" in categories of log schemes.

We suppose that we are in the situation of [4], §2. That is to say, let $X^{\log }$ be a locally noetherian $f_{s} \log$ scheme [cf. the discussion entitled "Log schemes" in $\S 0]$. Then we denote by

$$
\operatorname{Sch}^{\log }\left(X^{\log }\right)
$$

the category whose objects are morphisms of log schemes of finite type $Y^{\log } \rightarrow$ $X^{\log }$, where $Y^{\log }$ is a noetherian $f s \log$ scheme, and whose morphisms [from 
an object $Y_{1}^{\log } \rightarrow X^{\log }$ to an object $\left.Y_{2}^{\log } \rightarrow X^{\log }\right]$ are morphisms of finite type $Y_{1}^{\log } \rightarrow Y_{2}^{\log }$ lying over $X^{\log }$. To simplify the exposition, we shall often refer to the domain $Y^{\log }$ of an arrow $Y^{\log } \rightarrow X^{\log }$ which is an object of $\operatorname{Sch}^{\log }\left(X^{\log }\right)$ as an "object of $\operatorname{Sch}^{\log }\left(X^{\log }\right)$ ".

Recall the category $\operatorname{Sch}(X)$ of [4], $\S 1$, i.e., the category whose objects are morphisms of finite type $Y \rightarrow X$, where $Y$ is a noetherian scheme, and whose morphisms [from an object $Y_{1} \rightarrow X$ to an object $Y_{2} \rightarrow X$ ] are morphisms of finite type $Y_{1} \rightarrow Y_{2}$ lying over $X$. Note that by associating to an object $Y \rightarrow X$ of $\operatorname{Sch}(X)$ the object $Y^{\log } \rightarrow X^{\log }$ of $\operatorname{Sch}^{\log }\left(X^{\log }\right)$ obtained by equipping $Y$ with the $\log$ structure obtained by pulling back the $\log$ structure on $X^{\log }$ via $Y \rightarrow X$, we obtain a natural embedding

$$
\operatorname{Sch}(X) \hookrightarrow \operatorname{Sch}^{\log }\left(X^{\log }\right)
$$

-which thus allows us to regard $\operatorname{Sch}(X)$ as a full subcategory of $\operatorname{Sch}^{\log }\left(X^{\log }\right)$.

Let $Y^{\log }$ be an $f_{S} \log$ scheme. Then we shall denote its underlying scheme (respectively, the morphism of monoids that constitutes its log structure) by $Y$ (respectively, $\exp _{Y}: M_{Y} \rightarrow \mathcal{O}_{Y}$ ). Thus, we have an exact sequence of étale sheaves of monoids on $Y$

$$
0 \rightarrow \mathcal{O}_{Y}^{\times} \rightarrow M_{Y} \rightarrow P_{Y} \rightarrow 0
$$

-where the "characteristic sheaf" $P_{Y}$ is defined so as to make the sequence exact. It follows immediately from the fact that $Y^{\log }$ is an fs $\log$ scheme that the fibers of $P_{Y}$ (respectively, the groupification $P_{Y}^{\mathrm{gp}}$ of $P_{Y}$ ) at geometric points of $Y$ are $f s$ monoids [cf. the discussion entitled "Generalities on monoids" in $\$ 0$ ] (respectively, are finitely generated free abelian groups). In particular, we have natural injections

$$
P_{Y} \hookrightarrow P_{Y}^{\mathrm{gp}} ; \quad M_{Y} \hookrightarrow M_{Y}^{\mathrm{gp}}
$$

-where the superscript "gp" denotes the groupification associated to a sheaf of monoids. In the following, we shall use similar notation for objects associated to arbitrary fs $\log$ schemes " $(-)^{\log , "}$.

In this situation, we shall apply the terminology introduced in [4], §2:

Definition 1.1. In the notation of the above discussion:

(i) If $Y$ is reduced (respectively, one-pointed - cf. [4], Proposition 1.1), then we shall say that $Y^{\log }$ is reduced (respectively, one-pointed). If $Y^{\log }$ is reduced and one-pointed, i.e., $Y$ is equal to the spectrum of a field $k$, then one may think of $P_{Y}$ as consisting of a [discrete] monoid equipped with a continuous action of the absolute Galois group $G_{k}$ of $k$; when this action is trivial, we shall say that $Y^{\log }$ is split and, by a slight abuse of notation, denote $\Gamma\left(Y, P_{Y}\right)$ by $P_{Y}$.

(ii) An object $Y^{\log } \rightarrow X^{\log }$ of $\operatorname{Sch}^{\log }\left(X^{\log }\right)$ will be called minimal if it is non-initial and satisfies the property that any monomorphism $Z^{\log } \rightarrow Y^{\log }$ in $\operatorname{Sch}^{\log }\left(X^{\log }\right)$, where $Z^{\log }$ is non-initial, is necessarily an isomorphism [cf. [4], Proposition 2.4]. 
(iii) Suppose that $Y^{\log }$ is a one-pointed object of the category $\operatorname{Sch}^{\log }\left(X^{\log }\right)$. Then a monomorphism $H^{\log } \hookrightarrow Y^{\log }$ in $\operatorname{Sch}^{\log }\left(X^{\log }\right)$ will be called a hull for $Y^{\log }$ if every morphism $S^{\log } \rightarrow Y^{\log }$ in $\operatorname{Sch}{ }^{\log }\left(X^{\log }\right)$ from a minimal object $S^{\log }$ to $Y^{\log }$ factors [necessarily uniquely!] through the given monomorphism $H^{\log } \leadsto Y^{\log }$ [cf. [4], Proposition 2.7]. A hull $H^{\log } \mapsto Y^{\log }$ will be called a minimal hull if every monomorphism $H_{1}^{\log } \hookrightarrow H^{\log }$ in $\operatorname{Sch}^{\log }\left(X^{\log }\right)$ for which the composite $H_{1}^{\log } \longmapsto H^{\log } \hookrightarrow Y^{\log }$ is a hull is necessarily an isomorphism [cf. [4], Proposition 2.7]. A one-pointed object $H^{\log }$ of $\operatorname{Sch}^{\log }\left(X^{\log }\right)$ will be called a minimal hull if the identity morphism $H^{\log } \rightarrow H^{\log }$ is a minimal hull for $H^{\log }$. [The notions of "hull"/"minimal hull" will not be used in the present paper, but are reviewed here for the sake of comparison with the notions of "point-hull"/"minimal point-hull", which do play an important role in the present paper-cf. Definition 2.9, (iii).

(iv) Suppose that $f^{\log }: Z^{\log } \rightarrow Y^{\log }$ is a morphism of $\operatorname{Sch}^{\log }\left(X^{\log }\right)$. Then [cf. [4], Definition 2.11, (i), (ii)]: $f^{\log }$ will be called log-like if the underlying morphism of schemes $f: Z \rightarrow Y$ is an isomorphism; $f^{\log }$ will be called schemelike if the $\log$ structure on $Z^{\log }$ is the pull-back of the $\log$ structure on $Y^{\log }$ via the underlying morphism of schemes $f: Z \rightarrow Y$ [i.e., in the terminology of many authors, if $f^{\log }$ is strict]. Write

$$
\left.\operatorname{Sch}^{\log }\left(X^{\log }\right)\right|_{\text {sch-lk }} \subseteq \operatorname{Sch}^{\log }\left(X^{\log }\right)
$$

for the full subcategory of objects of $\operatorname{Sch}^{\log }\left(X^{\log }\right)$ determined by scheme-like morphisms $Y^{\log } \rightarrow X^{\log }$. Thus, one verifies immediately that the natural embed$\operatorname{ding} \operatorname{Sch}(X) \hookrightarrow \operatorname{Sch}^{\log }\left(X^{\log }\right)$ discussed above admits a natural factorization as the composite of a natural equivalence of categories

$$
\left.\operatorname{Sch}(X) \stackrel{\sim}{\rightarrow} \operatorname{Sch}^{\log }\left(X^{\log }\right)\right|_{\text {sch-lk }}
$$

with the natural inclusion $\left.\operatorname{Sch}^{\log }\left(X^{\log }\right)\right|_{\text {sch-lk }} \hookrightarrow \operatorname{Sch}^{\log }\left(X^{\log }\right)$.

Also, we introduce some new terminology as follows:

DEFINITION 1.2. In the notation of the above discussion:

(i) Let $n \in \mathbf{N}$. Then we shall say that $Y^{\log }$ is of rank $\leq n$ (respectively, of rank $n$ ) and write

$$
\operatorname{rk}\left(Y^{\log }\right) \leq n \quad\left(\text { respectively, } \operatorname{rk}\left(Y^{\log }\right)=n\right)
$$

if every fiber of $P_{Y}$ at a geometric point of $Y$ is of rank $\leq n$ (respectively, rank $n$ ) [cf. the discussion entitled "Generalities on monoids" in $§ 0$ ]. We shall say that $Y^{\log }$ is submonic if it is of rank $\leq 1$. If $Y^{\log }$ is locally noetherian, then we define the submonic dimension of $Y^{\log }$ to be the supremum

$$
\operatorname{dim}^{\operatorname{sm}}\left(Y^{\log }\right) \stackrel{\text { def }}{=} \sup _{Z^{\log } \mapsto Y^{\log }} \operatorname{dim}(Z) \in \mathbf{N} \cup\{-\infty,+\infty\}
$$

- where $Z^{\log } \hookrightarrow Y^{\log }$ ranges over the monomorphisms of $\operatorname{Sch}^{\log }\left(Y^{\log }\right)$ such that $Z^{\log }$ is submonic, and " $\operatorname{dim}(Z)$ " denotes the scheme-theoretic dimension of the 
underlying locally noetherian scheme $Z$ of $Z^{\log }$. Thus, the submonic dimension is equal to $-\infty$ if and only if it holds that the underlying scheme of every " $Z$ log" that appears in the supremum of the above display is the empty scheme. We shall say that $Y^{\log }$ is log-nodal if it is reduced, one-pointed, split, and of rank two.

(ii) Suppose that $Y^{\log }$ arises from an object $Y^{\log } \rightarrow X^{\log }$ of $\operatorname{Sch}^{\log }\left(X^{\log }\right)$. Then a minimal point $Z^{\log } \mapsto Y^{\log }$ of $Y^{\log }$ is defined to be a monomorphism $Z^{\log } \mapsto Y^{\log }$ of $\operatorname{Sch}^{\log }\left(X^{\log }\right)$ such that $Z^{\log }$ is a minimal object of $\operatorname{Sch}^{\log }\left(X^{\log }\right)$. Thus, a minimal point of $Y^{\log }$ may be thought of as an object of $\operatorname{Sch}^{\log }\left(Y^{\log }\right)$. We shall write

$$
\operatorname{MinPt}\left(Y^{\log }\right)
$$

for the set of isomorphism classes [i.e., as objects of $\operatorname{Sch}^{\log }\left(Y^{\log }\right)$ ] of minimal points of $Y^{\log }$.

Proposition 1.3 (Empty and connected underlying schemes). Suppose that $Y^{\log }$ is an object of $\operatorname{Sch}^{\log }\left(X^{\log }\right)$. Then:

(i) The underlying scheme $Y$ of $Y^{\log }$ is empty if and only if $Y^{\log }$ is an initial object in the category $\operatorname{Sch}^{\log }\left(X^{\log }\right)$.

(ii) The underlying scheme $Y$ of $Y^{\log }$ is connected if and only if the object $Y^{\log }$ of $\operatorname{Sch}^{\log }\left(X^{\log }\right)$ is non-initial and, moreover, does not admit a representation as a coproduct of two non-initial objects of $\operatorname{Sch}^{\log }\left(X^{\log }\right)$.

Proof. Assertions (i) and (ii) follow immediately from the definitions.

Proposition 1.4 (First properties of monomorphisms). Suppose that $f^{\log }$ : $Z^{\log } \rightarrow Y^{\log }$ is a morphism of $\operatorname{Sch}^{\log }\left(X^{\log }\right)$. Thus, the underlying morphism $f: Z \rightarrow Y$ of $f^{\log }$ may be regarded as a morphism of $\operatorname{Sch}(X)$. Then:

(i) The property of being a monomorphism in the category of $f_{s} \log$ schemes (respectively, in the category $\mathrm{Sch}^{\log }\left(X^{\log }\right)$ ) is stable under base-change in the category of $f s \log$ schemes (respectively, in the category $\operatorname{Sch}^{\log }\left(X^{\log }\right)$ ).

(ii) Let $M \rightarrow N$ be a morphism of finitely generated, saturated monoids such that the induced morphism $M^{\mathrm{gp}} \rightarrow N^{\mathrm{gp}}$ is surjective. Then the induced morphism of $f_{s} \log$ schemes

$$
\operatorname{Spec}(\mathbf{Z}[N])^{\log } \rightarrow \operatorname{Spec}(\mathbf{Z}[M])^{\log }
$$

— where we use the superscript "log" to denote the log structures determined by the tautological charts $M \hookrightarrow \mathbf{Z}[M], N \hookrightarrow \mathbf{Z}[N]$ - is a monomorphism in the category of $f_{s} \log$ schemes.

(iii) If $f^{\log }$ is a monomorphism in $\operatorname{Sch}^{\log }\left(X^{\log }\right)$, then the induced morphism of sheaves of abelian groups $\left.P_{Y}^{\mathrm{gp}}\right|_{Z} \rightarrow P_{Z}^{\mathrm{gp}}$ is surjective.

(iv) Suppose that $Y^{\log }$ is submonic, and that the morphism $\left.P_{Y}^{\mathrm{gp}}\right|_{Z} \rightarrow P_{Z}^{\mathrm{gp}}$ induced by $f^{\log }$ is surjective. Then $Z^{\log }$ is submonic, and $f^{\log }$ is scheme-like.

(v) Suppose that $f^{\log }$ is scheme-like. Then $f^{\log }$ is a monomorphism in $\operatorname{Sch}^{\log }\left(X^{\log }\right)$ if and only if $f$ is a monomorphism in $\operatorname{Sch}(X)$.

(vi) Suppose that $Y^{\log }$ is submonic, and that $f^{\log }$ is a monomorphism in $\operatorname{Sch}^{\log }\left(X^{\log }\right)$. Then the morphism $\left.P_{Y}^{\mathrm{gp}}\right|_{Z} \rightarrow P_{Z}^{\mathrm{gp}}$ induced by $f^{\log }$ is surjective; $Z^{\log }$ 
is submonic; $f^{\log }$ is scheme-like [which, in fact, implies the surjectivity of the morphism $\left.\left.P_{Y}^{\mathrm{gp}}\right|_{Z} \rightarrow P_{Z}^{\mathrm{gp}}\right]$; and $f$ is a monomorphism in $\operatorname{Sch}(X)$.

(vii) Suppose that $f$ is a monomorphism in $\operatorname{Sch}(X)$, and that the morphism $\left.P_{Y}^{\mathrm{gp}}\right|_{Z} \rightarrow P_{Z}^{\mathrm{gp}}$ induced by $f^{\log }$ is surjective. Then $f^{\log }$ is a monomorphism in $\operatorname{Sch}^{\log }\left(X^{\log }\right)$.

Proof. Assertions (i) and (v) follow immediately from the definitions. Next, before proceeding, let us recall that, for instance in the case of the log scheme $Y^{\log }$

$\left(*_{\text {sys }}\right)$ the sheaf of monoids that defines the log structure of $Y^{\log }$ may be thought of as the restriction to $P_{Y} \subseteq P_{Y}^{\mathrm{gp}}$ of a certain system of line bundles [i.e., a system of $\mathbf{G}_{\mathrm{m}}$-torsors] parametrized by the sheaf of abelian groups $P_{Y}^{\mathrm{gp}}$.

Now assertion (ii) follows immediately from $\left(*_{\text {sys }}\right)$. Assertion (iii) follows from the argument given in the proof of [4], Proposition 2.3 [but cf. Remark 1.4.1 below!]: That is to say, one reduces immediately to the case where $Z$ and $Y$ are equal to $\operatorname{Spec}(k)$ for some field $k$; then, under the assumption that the asserted surjectivity fails to hold, one constructs scheme-like morphisms $W^{\log } \rightarrow Z^{\log }$, where $W^{\log }$ is an fs log scheme whose underlying scheme is an artinian $k$-algebra, whose existence contradicts the assumption that $f^{\log }$ is a monomorphism in $\operatorname{Sch}^{\log }\left(X^{\log }\right)$. Assertion (iv) follows immediately from the simple and wellunderstood structure of the monoid $\mathbf{N}$. Assertion (vi) follows formally from assertions (iii), (iv), and (v). Finally, assertion (vii) follows from the definitions, together with the observation $\left(*_{\text {sys }}\right)$ discussed above.

Remark 1.4.1. Suppose that we are in the situation of Proposition 1.4. Then in general,

it is not necessarily the case that the assumption that $f^{\log }$ is a monomorphism in $\mathrm{Sch}^{\log }\left(X^{\log }\right)$ implies that $f$ is a monomorphism in $\operatorname{Sch}(X)$.

That is to say, the corresponding portion of the necessity asserted in [4], Proposition 2.3, is false as stated. Such an example may be obtained by considering the monomorphism constructed in Proposition 1.4, (ii), in the case where the morphism of monoids $M \rightarrow N$ is taken to be the morphism

$$
M \stackrel{\text { def }}{=} \mathbf{N} \oplus \mathbf{N} \rightarrow N \stackrel{\text { def }}{=} \mathbf{N} \oplus \mathbf{N}
$$

that maps $M \ni(1,0) \mapsto(1,1) \in N$ and $M \ni(0,1) \mapsto(0,1) \in N$, i.e., in which case the resulting morphism of schemes is a "blow-up morphism" that has fibers of dimension one.

LEMMA 1.5 (Well-known generalities concerning fs monoids and associated $\log$ schemes). Let $k$ be $a$ field; $k^{\text {sep }} a$ separable closure of $k ; G_{k} \stackrel{\text { def }}{=} \operatorname{Gal}\left(k^{\text {sep }} / k\right) ; P$ 
an fs monoid [cf. the discussion entitled "Generalities on monoids" in §0] equipped with a continuous action by $G_{k}[$ i.e., relative to the discrete topology on $P] ; M^{\mathrm{gp}}$ an extension, in the category of topological abelian groups equipped with continuous $G_{k}$-actions, of $P^{\mathrm{gp}}$ by $\left(k^{\mathrm{sep}}\right)^{\times}$[i.e., the multiplicative group of nonzero elements of $k^{\mathrm{sep}}$, equipped with the discrete topology]; $M \stackrel{\text { def }}{=} M^{\mathrm{gp}} \times_{P \mathrm{gp}} P . \quad$ Write $T^{\log }$ for the reduced, one-pointed $f_{s} \log$ scheme whose underlying scheme is equal to $T=$ $\operatorname{Spec}\left(k^{\mathrm{sep}}\right)$, and whose log structure is given by the homomorphism of monoids $M \rightarrow k^{\text {sep }}$ that restricts to the natural inclusion $\left(k^{\text {sep }}\right)^{\times} \hookrightarrow k^{\text {sep }}$ on $\left(k^{\text {sep }}\right)^{\times} \subseteq M$ and maps non-invertible elements of $M$ to $0 \in k$. Thus, the associated characteristic sheaf $P_{T}$ is the constant sheaf on $T$ determined by $P$; the log scheme $T^{\log }$ admits a natural $G_{k}$-action, which may be regarded as a collection of [pro-] finite étale descent data that gives rise to a reduced, one-pointed $f_{s} \log$ scheme $S^{\log }$ whose underlying scheme is $S=\operatorname{Spec}(k)$. Then:

(i) Suppose that the action of $G_{k}$ on $P$ is trivial. Then the extension of $G_{k^{-}}$ modules $1 \rightarrow\left(k^{\mathrm{sep}}\right)^{\times} \rightarrow M^{\mathrm{gp}} \rightarrow P^{\mathrm{gp}} \rightarrow 1$ splits.

(ii) Suppose that $\operatorname{rk}(P) \geq 1$. Then there exists a positive [cf. the discussion entitled "Generalities on monoids" in §0], $G_{k}$-equivariant [i.e., with respect to the trivial action of $G_{k}$ on $\left.\mathbf{N}\right]$ homomorphism $\phi: P \rightarrow \mathbf{N}$ that induces a surjection on groupifications $\phi^{\mathrm{gp}}: P^{\mathrm{gp}} \rightarrow \mathbf{N}^{\mathrm{gp}}$. Now fix such a homomorphism $\phi: P \rightarrow \mathbf{N}$, and assume, moreover, that $\operatorname{rk}(P) \geq 2$. Then there exists a positive homomorphism $\psi: P \rightarrow \mathbf{N}$ that induces a surjection on groupifications $\psi^{\mathrm{gp}}: P^{\mathrm{gp}} \rightarrow \mathbf{N}^{\mathrm{gp}}$ such that $\operatorname{Ker}\left(\phi^{\mathrm{gp}}\right) \neq \operatorname{Ker}\left(\psi^{\mathrm{gp}}\right)$.

(iii) Suppose that $\operatorname{rk}(P) \geq 2$. Then there exist an fs monoid $Q$ of rank two and $a$ positive homomorphism $\xi: P \rightarrow Q$ that induces a surjection on groupifications $\xi^{\mathrm{gp}}: P^{\mathrm{gp}} \rightarrow Q^{\mathrm{gp}}$, and, moreover, satisfies the following property:

Let $\zeta: Q \rightarrow R$ be a positive homomorphism of $f_{s}$ monoids of rank $\geq 1$ and $\sigma \in G_{k}$ such that the composite homomorphism $\zeta \circ \xi \circ \sigma: P \rightarrow R$ factors as the composite $\zeta_{\sigma} \circ \xi$ of $\xi: P \rightarrow Q$ with some positive homomorphism $\zeta_{\sigma}: Q \rightarrow R$. Then $\sigma$ stabilizes the subquotient $P^{\mathrm{gp}} \rightarrow Q^{\mathrm{gp}} \supseteq Q$ and induces the identity on $Q$.

In particular, if $\tau \in G_{k}$ stabilizes the subquotient $P^{\mathrm{gp}} \rightarrow Q^{\mathrm{gp}} \supseteq Q$, then $\tau$ induces the identity on $Q$.

(iv) Let $\xi: P \rightarrow Q$ be a positive homomorphism of $f s$ monoids that induces a surjection on groupifications $\xi^{\mathrm{gp}}: P^{\mathrm{gp}} \rightarrow Q^{\mathrm{gp}}$. Write $\Xi^{\mathrm{sep}}$ for the subfunctor of the contravariant functor determined by the terminal object $\left[i . e ., T^{\log }\right]$ of $\operatorname{Sch}^{\log }\left(T^{\log }\right)$ that consists of objects $Z^{\log } \rightarrow T^{\log }$ of $\operatorname{Sch}^{\log }\left(T^{\log }\right)$ such that the composite homomorphism $P^{\mathrm{gp}} \rightarrow \Gamma\left(T, P_{T}^{\mathrm{gp}}\right) \rightarrow \Gamma\left(Z, P_{Z}^{\mathrm{gp}}\right)$ induces, via $\xi$, a homomorphism $Q \rightarrow \Gamma\left(Z, P_{Z}\right)$; write $\Xi_{+}^{\text {sep }} \subseteq \Xi^{\text {sep }}$ for the subfunctor corresponding to the condition that, for each fiber $P_{Z, \bar{z}}$ of $P_{Z}$ at a geometric point $\bar{z}$ of $Z$, the resulting homomorphism $Q \rightarrow P_{Z, \bar{z}}$ is positive. Then $\Xi^{\text {sep }}$ may be represented by the object of $\operatorname{Sch}^{\log }\left(T^{\log }\right)$ determined by a log étale monomorphism

$$
T^{\log }[\xi] \nrightarrow T^{\log }
$$


of $\operatorname{Sch}^{\log }\left(T^{\log }\right)$. If , moreover, $Q$ coincides with the saturation of the image of $\xi$ in $Q^{\mathrm{gp}}$, then the following properties hold: $\Xi_{+}^{\mathrm{sep}}=\Xi^{\mathrm{sep}}$; the closed subscheme $T[\xi]_{\mathrm{red}} \subseteq T[\xi][c f$. the discussion entitled "Log schemes" in §0] of the underlying scheme $T[\xi]$ of $T^{\log }[\xi]$ is a torus over $k^{\text {sep }}$ of $\operatorname{dimension} \operatorname{rk}(P)-\operatorname{rk}(Q)$; the characteristic sheaf $P_{T^{\log }[\xi]}$ is isomorphic to the constant sheaf on $T[\xi]$ determined by $Q$; if we write $M[\xi] \stackrel{\text { def }}{=} M^{\mathrm{gp}} \times_{P \mathrm{gp}} \operatorname{Ker}\left(\xi^{\mathrm{gp}}\right)$, then the group of invertible functions on the torus $T[\xi]_{\mathrm{red}}$ may be naturally identified with $M[\xi]$.

(v) Suppose that we are in the situation of (iv). Write $H \subseteq G_{k}$ for the open subgroup of elements that stabilize the subquotient $P^{\mathrm{gp}} \rightarrow Q^{\mathrm{gp}} \supseteq Q$ determined by $\xi ; S_{H}^{\log }$ for the reduced, one-pointed $f_{S} \log$ scheme obtained by descending $T^{\log }$ via $H \subseteq G_{k}$; $\Xi$ for the subfunctor of the contravariant functor determined by the terminal object $\left[\right.$ i.e., $S_{H}^{\log }$ ] of $\operatorname{Sch}^{\log }\left(S_{H}^{\log }\right)$ that consists of objects $Z^{\log } \rightarrow S_{H}^{\log }$ of $\operatorname{Sch}^{\log }\left(S_{H}^{\log }\right)$ such that the object $Z^{\log } \times{ }_{S_{H}^{\log }} T^{\log } \rightarrow T^{\log }$ of $\operatorname{Sch}^{\log }\left(T^{\log }\right)$ determined by base-changing from $S_{H}^{\log }$ to $T^{\log }$ determines an element of $\Xi^{\operatorname{sep}}\left(Z^{\log } \times_{S_{H}^{\log }} T^{\log }\right)$; $\Xi_{+}$for the subfunctor of $\Xi$ determined by the subfunctor $\Xi_{+}^{\text {sep }}$ of $\Xi^{\text {sep }}$. Then $\Xi$ may be represented by the object of $\operatorname{Sch}^{\log }\left(S_{H}^{\log }\right)$ determined by a log étale monomorphism

$$
S^{\log }[\xi] \mapsto S_{H}^{\log }
$$

of $\operatorname{Sch}^{\log }\left(S_{H}^{\log }\right)$ which may be obtained, via [pro-]finite étale descent, from the natural $H$-action on the monomorphism $T^{\log }[\xi] \mapsto T^{\log }$ of (iv).

(vi) Suppose that we are in the situation of $(\mathrm{v})$. Let $S_{+}^{\log }[\xi] \nrightarrow S^{\log }[\xi]$ be some monomorphism of $\operatorname{Sch}^{\log }\left(S_{H}^{\log }\right)$ that determines an element of $\Xi_{+}(-) \subseteq \Xi(-)$. [That is say, we do not make any assumption to the effect that $S_{+}^{\log }[\xi]$ admits some sort of "special functorial interpretation"!] Then if either $\operatorname{rk}(Q)=1$ or $\xi$ is as in (iii), then the composite

$$
S_{+}^{\log }[\xi] \mapsto S^{\log }[\xi] \longmapsto S_{H}^{\log } \rightarrow S^{\log }
$$

- where the second arrow is the monomorphism of the final display of (v); the third arrow is the natural morphism $S_{H}^{\log } \rightarrow S^{\log }$-is a monomorphism in $\operatorname{Sch}^{\log }\left(S^{\log }\right)$.

(vii) Suppose that $\operatorname{rk}(P)=2$, and that we have been given a positive homomorphism $\phi_{0}: P \rightarrow J_{0} \stackrel{\text { def }}{=} \mathbf{N}$ that induces a surjection on groupifications $\phi_{0}^{\mathrm{gp}}: P^{\mathrm{gp}} \rightarrow$ $J_{0}^{\mathrm{gp}}=\mathbf{Z}$. Then, in the notation of the discussion entitled "Rank two fs monoids" in $\S 0$, for $i=0,1,2$, let us write $\phi_{i}: P \rightarrow J_{i}$ for the associated positive homomorphism of $f_{s}$ monoids [which is well-defined, up to possible permutation of the indices "1" and "2"]. For $i=0,1,2$, write $\Phi_{i}^{\text {sep }}$ for the subfunctor of the contravariant functor determined by the terminal object [i.e., $\left.T^{\log }\right]$ of $\operatorname{Sch}^{\log }\left(T^{\log }\right)$ that consists of objects $Z^{\log } \rightarrow T^{\log }$ of $\mathrm{Sch}^{\log }\left(T^{\log }\right)$ such that, for each fiber $P_{Z, \bar{z}}$ of $P_{Z}$ at a geometric point $\bar{z}$ of $Z$, the composite homomorphism $P^{\mathrm{gp}} \rightarrow \Gamma\left(T, P_{T}^{\mathrm{gp}}\right) \rightarrow$ $\Gamma\left(Z, P_{Z}^{\mathrm{gp}}\right) \rightarrow P_{Z, \bar{z}}^{\mathrm{gp}}$ induces, via $\phi_{i}: P \rightarrow J_{i}$, a positive homomorphism $J_{i} \rightarrow P_{Z, \bar{z}}$. If $E \subseteq\{0,1,2\}$ is a subset, then write $\Phi_{E}^{\mathrm{sep}}$ for the subfunctor of the contravariant functor determined by the terminal object [i.e., $\left.T^{\log }\right]$ of $\operatorname{Sch}^{\log }\left(T^{\log }\right)$ that consists of the [disjoint!] union of the $\Phi_{i}^{\text {sep }}$, for $i \in E$. Then, for any $E \subseteq\{0,1,2\}$ such 
that $0 \in E, \Phi_{E}^{\text {sep }}$ may be represented by the object of $\operatorname{Sch}^{\log }\left(T^{\log }\right)$ determined by $a$ log étale monomorphism

$$
T^{\log }\left[\phi_{E}\right] \longmapsto T^{\log }
$$

of $\operatorname{Sch}^{\log }\left(T^{\log }\right)$ which satisfies the following properties: $T^{\log }\left[\phi_{E}\right]$ is connected [hence nonempty]. If $E=\{0\}$, then $T^{\log }\left[\phi_{E}\right] \mapsto T^{\log }$ may be identified with the morphism $T^{\log }\left[\phi_{0}\right] \mapsto T^{\log }$ of (iv); in particular, in this case, the closed subscheme $T\left[\phi_{E}\right]_{\mathrm{red}} \subseteq T\left[\phi_{E}\right]$ of the underlying scheme $T\left[\phi_{E}\right]$ of $T^{\log }\left[\phi_{E}\right]$ is a one-dimensional torus over $k^{\mathrm{sep}}$. Finally, if $0 \in E \subseteq E^{*} \subseteq\{0,1,2\}$, then the resulting morphism of $\log$ schemes $T^{\log }\left[\phi_{E}\right] \rightarrow T^{\log }\left[\phi_{E^{*}}\right]$ is a dominant open immersion [cf. the discussion entitled "Log schemes" in §0].

(viii) Suppose that we are in the situation of (vii). Write $H \subseteq G_{k}$ for the open subgroup of elements that stabilize the subquotient $P^{\mathrm{gp}} \rightarrow J_{0}^{\mathrm{gp}} \supseteq J_{0}$ determined by $\phi_{0} ; S_{H}^{\log }$ for the reduced, one-pointed $f_{S} \log$ scheme obtained by descending $T^{\log }$ via $H \subseteq G_{k}$. Thus, $H$ acts naturally on Prime $(P)$, hence also on the set of indices $\{0,1,2\}$ [where we regard the index " 0 " as being stabilized by the action of $H]$. Let $E \subseteq\{0,1,2\}$ be a subset that is stabilized by this natural action of $H$. Write $\Phi_{E}$ for the subfunctor of the contravariant functor determined by the terminal object $\left[\right.$ i.e., $\left.S_{H}^{\log }\right]$ of $\operatorname{Sch}^{\log }\left(S_{H}^{\log }\right)$ that consists of objects $Z^{\log } \rightarrow S_{H}^{\log }$ of $\operatorname{Sch}^{\log }\left(S_{H}^{\log }\right)$ such that the object $Z^{\log } \times_{S_{H} \log } T^{\log } \rightarrow T^{\log }$ of $\operatorname{Sch}^{\log }\left(T^{\log }\right)$ determined by base-changing from $S_{H}^{\log }$ to $T^{\log }$ determines an element of $\Phi_{E}^{\mathrm{sep}}\left(Z^{\log } \times_{S_{H}^{\log }} T^{\log }\right)$. Suppose that $0 \in E$. Then $\Phi_{E}$ may be represented by the object of $\operatorname{Sch}^{\log }\left(S_{H}^{\log }\right)$ determined by a log étale monomorphism

$$
S^{\log }\left[\phi_{E}\right] \longmapsto S_{H}^{\log }
$$

of $\operatorname{Sch}^{\log }\left(S_{H}^{\log }\right)$ which may be obtained, via [pro-]finite étale descent, from the natural $H$-action on the monomorphism $T^{\log }\left[\phi_{E}\right] \mapsto T^{\log }$ of (vii).

(ix) Suppose that we are in the situation of (viii). Suppose further that $\phi_{0}: P \rightarrow J_{0}$ satisfies the following property:

Let $\zeta: P \rightarrow \mathbf{N}$ be a positive homomorphism of $f_{s}$ monoids; $\sigma \in G_{k}$; $i_{0}, i_{1} \in\{0,1\}$. Suppose that, for $m \in\{0,1\}, \zeta \circ \sigma^{m}: P \rightarrow \mathbf{N}$ factors, via $\phi_{i_{m}}: P \rightarrow J_{i_{m}}$, through a positive homomorphism $J_{i_{m}} \rightarrow \mathbf{N}$. Then $\sigma$ acts trivially on $P$.

Then [one verifies immediately, by taking " $\zeta$ " to be $\phi_{0}$ that] $H$ fixes the index "1". Moreover, the composite $S^{\log }\left[\phi_{\{0,1\}}\right] \mapsto S_{H}^{\log } \rightarrow S^{\log }$ of the monomorphism of the final display of (viii) with the natural morphism $S_{H}^{\log } \rightarrow S^{\log }$ is a monomorphism in $\operatorname{Sch}^{\log }\left(S^{\log }\right)$.

Proof. Since $P^{\mathrm{gp}}$ is a finitely generated free abelian group, assertion (i) follows immediately from the assumption that the action of $G_{k}$ on $P$ is trivial, together with the well-known fact from elementary Galois theory [i.e., Hilbert's 
"Theorem 90"] that $H^{1}\left(G_{k},\left(k^{\text {sep }}\right)^{\times}\right)=0$. Next, we consider assertion (ii). The existence of $\phi$ follows immediately from [4], Lemma 2.5, (iii), i.e., by considering the [finite!] sum of the $G_{k}$-conjugates of a positive homomorphism $P \rightarrow \mathbf{N}$ of the sort discussed in [4], Lemma 2.5, (iii); the existence of $\psi$ then follows by applying [4], Lemma 2.5, (iii), to two distinct elements of $P$ that map, via $\phi$, to the same nonzero element of $\mathbf{N}$. This completes the proof of assertion (ii).

Next, we consider assertion (iii). First, we observe that the final portion of assertion (iii) concerning $\tau \in G_{k}$ follows immediately from the property in the display of assertion (iii) by taking $\zeta: Q \rightarrow R$ to be the identity automorphism of $Q$. Next, we observe that the homomorphisms $\phi$ and $\psi$ of assertion (ii) determine a positive homomorphism $(\phi, \psi): P \rightarrow \mathbf{N} \oplus \mathbf{N}$ whose image $I \subseteq \mathbf{N} \oplus \mathbf{N}$ generates a rank two subgroup $I^{\mathrm{gp}}$ of $\mathbf{N}^{\mathrm{gp}} \oplus \mathbf{N}^{\mathrm{gp}}=\mathbf{Z} \oplus \mathbf{Z}$. Thus, for some positive integer $n$, it holds that $n \cdot \mathbf{N}^{\mathrm{gp}} \oplus n \cdot \mathbf{N}^{\mathrm{gp}} \subseteq I^{\mathrm{gp}}$. In particular, we have $n \cdot \mathbf{N} \oplus n \cdot \mathbf{N} \subseteq$ $Q \stackrel{\text { def }}{=} I^{\mathrm{gp}} \cap(\mathbf{N} \oplus \mathbf{N}) \subseteq \mathbf{N} \oplus \mathbf{N} ; \quad I \subseteq Q ; \quad Q^{\mathrm{gp}}=I^{\mathrm{gp}} \quad\left[\right.$ since $\left.I^{\mathrm{gp}} \subseteq Q^{\mathrm{gp}} \subseteq I^{\mathrm{gp}}\right] . \quad$ One verifies immediately that this implies that this monoid $Q \subseteq \mathbf{N} \oplus \mathbf{N}$ is an $f_{S}$ monoid of rank two. Write $\xi: P \rightarrow Q$ for the resulting positive homomorphism of monoids. Note that $\xi$ induces a surjection on groupifications $\xi^{\mathrm{gp}}: P^{\mathrm{gp}} \rightarrow$ $Q^{\mathrm{gp}}\left(=I^{\mathrm{gp}}\right)$.

Now suppose that $\zeta: Q \rightarrow R$ is a positive homomorphism of fs monoids of rank $\geq 1$ and $\sigma \in G_{k}$ such that the composite homomorphism $\zeta \circ \xi \circ \sigma: P \rightarrow R$ factors as the composite $\zeta_{\sigma} \circ \xi$ of $\xi: P \rightarrow Q$ with some positive homomorphism $\zeta_{\sigma}: Q \rightarrow R$; in the following, we shall show that $\sigma$ stabilizes the subquotient $P^{\mathrm{gp}} \rightarrow Q^{\mathrm{gp}} \supseteq Q$ and induces the identity on $Q$. Here, we note that, by applying assertion (ii) in the case where we take " $P$ ", " $k$ ", and " $M$ "gp" to be $R, k^{\text {sep }}$, and $R^{\mathrm{gp}} \times\left(k^{\mathrm{sep}}\right)^{\times}$, respectively, we may assume without loss of generality that $R=\mathbf{N}$. Also, by replacing $R$ by a suitable submonoid of $R$, we may assume without loss of generality that $\zeta, \zeta_{\sigma}$ induce surjections $\zeta^{\mathrm{gp}}, \zeta_{\sigma}^{\mathrm{gp}}: Q^{\mathrm{gp}} \rightarrow R^{\mathrm{gp}}=$ $\mathbf{N}^{\mathrm{gp}}=\mathbf{Z}$. Next, let us observe that, by restricting the first projection $\mathbf{N} \oplus \mathbf{N} \rightarrow$ $\mathbf{N}$ to $Q \subseteq \mathbf{N} \oplus \mathbf{N}$, one may regard $\phi: P \rightarrow \mathbf{N}$ as the composite $\eta \circ \xi$ of $\xi: P \rightarrow Q$ with a homomorphism of monoids $\eta: Q \rightarrow \mathbf{N}$. Since $\eta$ vanishes on $0 \oplus n \cdot \mathbf{N} \subseteq Q$, it follows that $\eta$ is not positive, and hence that $\operatorname{Ker}\left(\eta^{\mathrm{gp}}\right) \neq \operatorname{Ker}\left(\zeta^{\mathrm{gp}}\right), \operatorname{Ker}\left(\eta^{\mathrm{gp}}\right) \neq$ $\operatorname{Ker}\left(\zeta_{\sigma}^{\mathrm{gp}}\right)$. Since $\xi^{\mathrm{gp}}$ is surjective, we thus conclude that, if we write $\theta \stackrel{\text { def }}{=} \zeta \circ \xi$, $\theta_{\sigma} \stackrel{\text { def }}{=} \zeta_{\sigma} \circ \xi$, then $\operatorname{Ker}\left(\phi^{\mathrm{gp}}\right) \neq \operatorname{Ker}\left(\theta^{\mathrm{gp}}\right), \operatorname{Ker}\left(\phi^{\mathrm{gp}}\right) \neq \operatorname{Ker}\left(\theta_{\sigma}^{\mathrm{gp}}\right)$, and hence that both $\operatorname{Ker}\left(\phi^{\mathrm{gp}}\right) \cap \operatorname{Ker}\left(\theta^{\mathrm{gp}}\right) \subseteq P^{\mathrm{gp}}$ and $\operatorname{Ker}\left(\phi^{\mathrm{gp}}\right) \cap \operatorname{Ker}\left(\theta_{\sigma}^{\mathrm{gp}}\right) \subseteq P^{\mathrm{gp}}$ are submodules of $\operatorname{rank} \operatorname{rk}\left(P^{\mathrm{gp}}\right)-2$ that contain $\operatorname{Ker}\left(\xi^{\mathrm{gp}}\right)$. Since $\operatorname{Ker}\left(\xi^{\mathrm{gp}}\right)$ is also a submodule of $P^{\mathrm{gp}}$ of $\operatorname{rank} \operatorname{rk}\left(P^{\mathrm{gp}}\right)-2$, we thus conclude [since $P^{\mathrm{gp}} / \operatorname{Ker}\left(\xi^{\mathrm{gp}}\right) \stackrel{\sim}{\rightarrow} Q^{\mathrm{gp}}$ is torsion-free] that $\operatorname{Ker}\left(\phi^{\mathrm{gp}}\right) \cap \operatorname{Ker}\left(\theta^{\mathrm{gp}}\right)=\operatorname{Ker}\left(\phi^{\mathrm{gp}}\right) \cap \operatorname{Ker}\left(\theta_{\sigma}^{\mathrm{gp}}\right)=\operatorname{Ker}\left(\xi^{\mathrm{gp}}\right)$. But, since $\phi$ is $G_{k}$-equivariant, this implies that $\operatorname{Ker}\left(\xi^{\mathrm{gp}}\right)$ is stabilized by $\sigma$, i.e., that $\sigma$ induces an automorphism of the quotient $\xi^{\mathrm{gp}}: P^{\mathrm{gp}} \rightarrow Q^{\mathrm{gp}}$, as well as of the quotient $\eta^{\mathrm{gp}}: Q^{\mathrm{gp}} \rightarrow \mathbf{N}^{\mathrm{gp}}=\mathbf{Z}$, and maps the quotient $\zeta^{\mathrm{gp}}: Q^{\mathrm{gp}} \rightarrow \mathbf{N}^{\mathrm{gp}}=\mathbf{Z}$ to the quotient $\zeta_{\sigma}^{\mathrm{gp}}: Q^{\mathrm{gp}} \rightarrow \mathbf{N}^{\mathrm{gp}}=\mathbf{Z}$.

Now to complete the proof of assertion (iii), it suffices to verify that $\sigma$ induces the identity on $Q^{\mathrm{gp}}$. Thus, we suppose that $\sigma$ does not induce the identity on $Q^{\mathrm{gp}}$. Then since $\sigma$ clearly stabilizes the $f s$ monoid of rank two 
obtained by forming the saturation of the image of $\xi: P \rightarrow Q$ in $Q$ [cf. [4], Lemma 2.5, (ii)], it follows [cf. the discussion entitled "Rank two fs monoids" in $\S 0$ ] that $\sigma$ acts on $Q^{\text {gp }}$ as an automorphism of order 2, and hence that $\sigma$ permutes the quotients determined by $\zeta^{\mathrm{gp}}, \zeta_{\sigma}^{\mathrm{gp}}$. In particular, $\sigma$ stabilizes the kernel of the homomorphism on groupifications $\zeta_{+}^{\mathrm{gp}}: Q^{\mathrm{gp}} \rightarrow \mathbf{Z}$ determined by the positive homomorphism $\zeta_{+}: Q \rightarrow \mathbf{N}$ obtained by forming the sum of $\zeta, \zeta_{\sigma}$. Since $\sigma$ acts nontrivially on $Q^{\mathrm{gp}}$, this implies that $\operatorname{Ker}\left(\zeta_{+}^{\mathrm{gp}}\right)=\operatorname{Ker}\left(\eta^{\mathrm{gp}}\right)$. Thus, the positivity of $\zeta_{+}$contradicts the non-positivity of $\eta$. This completes the proof of assertion (iii).

Next, we observe that assertions (iv), (v), (vii), and (viii) are immediate consequences of the well-known correspondence between the theory of log schemes and the classical theory of toric varieties. Next, we consider assertion (vi). First of all, given an object $Z^{\log }$ of $\operatorname{Sch}^{\log }\left(S^{\log }\right)$ and two $S^{\log }$-morphisms $\alpha: Z^{\log } \rightarrow$ $S_{+}^{\log }[\xi], \beta: Z^{\log } \rightarrow S_{+}^{\log }[\xi]$, to verify that $\alpha=\beta$, it suffices to verify that $\alpha$ and $\beta$ coincide after base-change from $k$ to $k^{\text {sep }}$. Moreover, since the morphism $S_{H} \rightarrow S$ is finite étale, and the morphism $S_{+}^{\log }[\xi] \mapsto S_{H}^{\log }$ is already known to be a monomorphism, one verifies immediately that we may assume without loss of generality that $Z^{\log }$ is reduced and one-pointed - an assumption which reduces the assertion under consideration to an assertion concerning $f s$ monoids, i.e., the assertion that if, for some $\sigma \in G_{k}$, there exist positive homomorphisms of fs monoids $\zeta: Q \rightarrow R$ and $\zeta_{\sigma}: Q \rightarrow R$ such that $\zeta \circ \xi \circ \sigma=\zeta_{\sigma} \circ \xi: P \rightarrow R$, then $\sigma$ stabilizes the subquotient $P^{\mathrm{gp}} \rightarrow Q^{\mathrm{gp}} \supseteq Q$ determined by $\xi$. But this assertion concerning fs monoids follows immediately, i.e., if one assumes either that $\operatorname{rk}(Q)=1$ or that $\xi$ satisfies the properties stated in (iii). This completes the proof of assertion (vi). Finally, we observe that assertion (ix) may be verified by a similar argument to the argument applied in the proof of assertion (vi).

Proposition 1.6 (Minimal objects). Suppose that $Y^{\log }$ is an object of $\mathrm{Sch}^{\log }\left(X^{\log }\right)$. Then:

(i) Suppose that $Y^{\log }$ is a nonempty object of $\operatorname{Sch}^{\log }\left(X^{\log }\right)$. Then there exists a minimal point $Z^{\log } \rightarrow Y^{\log }$ such that $Z^{\log }$ is submonic. Now fix such a minimal point $Z^{\log } \rightarrow Y^{\log }$, and assume, moreover, that $Y^{\log }$ is not submonic. Then there exists a minimal point $W^{\log } \rightarrow Y^{\log }$, where $W^{\log }$ is submonic, that is not isomorphic to $Z^{\log } \rightarrow Y^{\log }$.

(ii) $Y^{\log }$ is a minimal object of $\operatorname{Sch}^{\log }\left(X^{\log }\right)$ if and only if $Y^{\log }$ is reduced, one-pointed, and submonic. Put another way, $Y^{\log }$ is a minimal object of $\operatorname{Sch}^{\log }\left(X^{\log }\right)$ if and only if, for some field $k, Y^{\log }$ is either equal to $\operatorname{Spec}(k)$ equipped with the trivial $\log$ structure or equal to $\operatorname{Spec}(k)$ equipped with the log structure $\mathbf{N} \ni 1 \mapsto 0 \in k$.

(iii) Suppose that $Y^{\log }$ and $Z^{\log }$ are minimal objects of $\operatorname{Sch}^{\log }\left(X^{\log }\right)$. If $f^{\log }: Z^{\log } \rightarrow Y^{\log }$ is a morphism in $\operatorname{Sch}^{\log }\left(X^{\log }\right)$, then let us write

$$
\operatorname{MinLg}\left(f^{\log }\right) \in \mathbf{N} \cup\{+\infty\}
$$

for the "minimal length" of $f^{\log }$ : that is to say, we set $\operatorname{MinL}\left(f^{\log }\right) \stackrel{\text { def }}{=} 0$ if $f^{\log }$ is an isomorphism; if $f^{\log }$ is not an isomorphism, then we take $\operatorname{Min} \operatorname{Lg}\left(f^{\log }\right)$ to 
be the supremum of the set of positive integers $n$ such that $f^{\log }$ admits a factorization

$$
Z_{n}^{\log \stackrel{\text { def }}{=}} Z^{\log } \rightarrow Z_{n-1}^{\log } \rightarrow \cdots \rightarrow Z_{1}^{\log } \rightarrow Z_{0}^{\log } \stackrel{\text { def }}{=} Y^{\log }
$$

as a composite of morphisms of $\operatorname{Sch}^{\log }\left(X^{\log }\right)$ which are not isomorphisms such that, for each $i=1, \ldots, n, Z_{i}^{\log }$ is a minimal object of $\operatorname{Sch}^{\log }\left(X^{\log }\right)$. Then $Y^{\log }$ is of rank one if and only if $\operatorname{MinLg}\left(f^{\log }\right)$ is finite for every morphism $f^{\log }$ in $\operatorname{Sch}^{\log }\left(X^{\log }\right)$ with codomain equal to $Y^{\log }$ [and domain given by some minimal object].

Proof. First, we consider assertion (i). Observe that we may assume without loss of generality that $Y^{\log }$ is reduced and one-pointed [cf. [4], Proposition 1.1, (i)], and hence that the underlying scheme $Y$ of $Y^{\log }$ may be written in the form $\operatorname{Spec}\left(k_{Y}\right)$, for a suitable field $k_{Y}$. Next, let us consider the situation discussed in Lemma 1.5, (v), in the case where

- one takes the data that gives rise to " $S^{\log }$ " to be the data that arises from $Y^{\log }$ [so " $k$ " corresponds to $k_{Y}$ ];

- if $\operatorname{rk}\left(Y^{\log }\right)=0$, then one takes the positive homomorphism " $\xi$ " to be the identity morphism;

- if $\operatorname{rk}\left(Y^{\log }\right) \geq 1$, then one takes the positive homomorphism " $\xi$ " to be the homomorphism " $\phi: P \rightarrow \mathbf{N}$ " of Lemma 1.5, (ii).

Then one verifies immediately from the description of the torus " $T[\xi]_{\mathrm{red}}$ " in Lemma 1.5, (iv), that any splitting as in Lemma 1.5, (i), over a suitable finite separable extension of $k$-which, in the terminology of [3], Definition 1.3, may be regarded as a "Galois-equivariant clean chart" - determines a closed point of $S[\xi]$. In particular, by restricting the log structure of the submonic log scheme $S^{\log }[\xi]$ to this closed point, we obtain, by Proposition 1.4, (vii); Lemma 1.5 , (vi), a monomorphism $f^{\log }: Z^{\log } \rightarrow Y^{\log }$ in $\operatorname{Sch}^{\log }\left(X^{\log }\right)$, for some submonic $Z^{\log }$. Since, by [4], Proposition 2.4, (ii), (iii), $Z^{\log }$ is necessarily minimal, we thus conclude that the morphism $f^{\log }$ determines a minimal point of $Y^{\log }$, as desired. In a similar vein, if $Y^{\log }$ is not submonic [i.e., is of rank $n \geq 2$ ], then we consider the situation discussed in Lemma 1.5, (v), in the case where one takes the data that gives rise to " $S$ log" to be the data that arises from $Y^{\log }$ [so " $k$ " corresponds to $k_{Y}$ ], and one takes the positive homomorphism " $\xi$ " to be the homomorphism " $\psi: P \rightarrow \mathbf{N}$ " of Lemma 1.5, (ii). Then a splitting as in Lemma 1.5 , (i), over a suitable finite separable extension of $k$ determines a closed point of $S[\xi]$ whose residue field $k_{W}$ is a finite separable extension field of $k_{Y}$. Now, by restricting the $\log$ structure of the submonic $\log$ scheme $S^{\log }[\xi]$ to this closed point, we obtain, by Proposition 1.4, (vii); Lemma 1.5, (vi), a monomorphism $W^{\log } \rightarrow Y^{\log }$ in $\operatorname{Sch}^{\log }\left(X^{\log }\right)$, for some submonic $W^{\log }$ whose underlying scheme $W$ is equal to $\operatorname{Spec}\left(k_{W}\right)$, which determines, by [4], Proposition 2.4, (iii), a minimal point of $Y^{\log }$ that is not isomorphic to $f^{\log }: Z^{\log } \rightarrow Y^{\log }$. This completes the proof of assertion (i). 
Next, we consider assertion (ii). The sufficiency portion of assertion (ii) follows immediately from [4], Proposition 2.4, (ii), (iii). Thus, to complete the proof of assertion (ii), it suffices to verify the necessity portion of assertion (ii). To this end, suppose that $Y^{\log }$ is minimal. Then it follows from [4], Proposition 2.4, (i), that $Y^{\log }$ is reduced and one-pointed, i.e., that $Y=\operatorname{Spec}\left(k_{Y}\right)$, for some field $k_{Y}$, and hence, from assertion (i), that there exists a minimal point $f^{\log }: Z^{\log } \rightarrow Y^{\log }$ in $\operatorname{Sch}^{\log }\left(X^{\log }\right)$, for some submonic $Z^{\log }$. If $Y^{\log }$ is not submonic, then it follows that $f^{\log }$ is not an isomorphism, i.e., in contradiction to the assumed minimality of $Y^{\log }$. This completes the proof of assertion (ii).

Finally, we consider assertion (iii). First, let us observe that it follows from assertion (ii) that the underlying scheme $Y$ (respectively, $Z$ ) of $Y^{\log }$ (respectively, $\left.Z^{\log }\right)$ may be written in the form $\operatorname{Spec}\left(k_{Y}\right)$ (respectively, $\operatorname{Spec}\left(k_{Z}\right)$ ), for a suitable field $k_{Y}$ (respectively, $k_{Z}$ ). Then if $Y^{\log }$ is of rank one, then the finiteness of $\operatorname{MinLg}\left(f^{\log }\right)$ follows immediately by considering the finiteness of the extension degree $\left[k_{Z}: k_{Y}\right]$, together with the simple, well-understood structure of the monoid $\mathbf{N}$. On the other hand, if $Y^{\log }$ is of rank zero, but $Z^{\log }$ is of rank one, then the fact that $\operatorname{MinLg}\left(f^{\log }\right)=+\infty$ follows by considering the infinite descending sequence of submonoids $\mathbf{N} \supseteq 2 \cdot \mathbf{N} \supseteq \cdots \supseteq 2^{n} \cdot \mathbf{N} \supseteq \cdots$, for $1 \leq n \in \mathbf{N}$. This completes the proof of assertion (iii).

Proposition 1.7 (Monomorphisms from log-nodal objects into non-submonic objects). Suppose that $Y^{\log }$ is a non-submonic object of $\operatorname{Sch}^{\log }\left(X^{\log }\right)$. Then there exists a log-nodal object $Z^{\log }$ of $\operatorname{Sch}^{\log }\left(X^{\log }\right)$ that admits a monomorphism $Z^{\log } \mapsto Y^{\log }$.

Proof. As in the proof of Proposition 1.6, (i), one verifies immediately that we may assume without loss of generality that $Y^{\log }$ is reduced and one-pointed, i.e., that $Y=\operatorname{Spec}\left(k_{Y}\right)$, for some field $k_{Y}$. Now we consider the situation discussed in Lemma 1.5, (v), in the case where one takes the data that gives rise

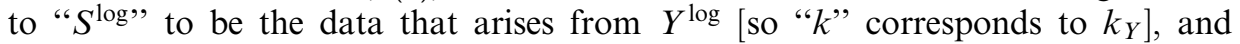
one takes the positive homomorphism " $\xi$ " to be the homomorphism " $\xi: P \rightarrow Q$ " of Lemma 1.5, (iii). Then one verifies immediately that any splitting as in Lemma 1.5, (i), over a suitable finite separable extension of $k$ determines a closed point of $S[\xi]$ whose residue field $k_{Z}$ is a finite separable extension field of $k_{Y}$ such that the $\log$ scheme $Z^{\log }$ obtained by restricting the log structure of the log scheme $S^{\log }[\xi]$ to this closed point determines an element of $\Xi_{+}(-) \subseteq \Xi(-)$. Thus, we obtain, by Proposition 1.4, (vii); Lemma 1.5, (vi), a monomorphism $Z^{\log } \rightarrow Y^{\log }$ in $\operatorname{Sch}^{\log }\left(X^{\log }\right)$, for some reduced, one-pointed, split [cf. the final portion of Lemma 1.5, (iii)] $Z^{\log }$ of rank two [cf. Lemma 1.5, (iii)] whose underlying scheme $Z$ is equal to $\operatorname{Spec}\left(k_{Z}\right)$, as desired.

Proposition 1.8 (Submonic one-pointed log schemes). Suppose that $Y^{\log }$ is an object of $\operatorname{Sch}^{\log }\left(X^{\log }\right)$. Then $Y^{\log }$ is submonic and one-pointed if and only if $\operatorname{MinPt}\left(Y^{\log }\right)$ is of cardinality one. 
Proof. First, we verify necessity. Suppose that $Y^{\log }$ is submonic and onepointed. Then it follows that $Y_{\text {red }}^{\log }$ [cf. the discussion entitled "Log schemes" in $\S 0]$ is reduced, one-pointed, and submonic, hence, by Proposition 1.6, (ii), that $Y_{\mathrm{red}}^{\log }$ is minimal. Since any morphism from a [necessarily reduced, by Proposition 1.6, (ii)!] minimal object of $\operatorname{Sch}^{\log }\left(X^{\log }\right)$ to $Y^{\log }$ clearly factors uniquely through $Y_{\text {red }}^{\log \text {, }}$ we thus conclude that $\operatorname{MinPt}\left(Y^{\log }\right)$ is of cardinality one, and that the unique element of $\operatorname{MinPt}\left(Y^{\log }\right)$ arises from the natural inclusion $Y_{\text {red }}^{\log } \hookrightarrow Y^{\log }$. This completes the proof of necessity. Next, we verify sufficiency. Suppose that $\operatorname{MinPt}\left(Y^{\log }\right)$ is of cardinality one. Then by applying the initial portion of

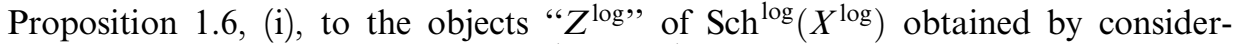
ing scheme-like monomorphisms $Z^{\log } \hookrightarrow Y^{\log }$ that arise from monomorphisms $Z \mapsto Y$ in $\operatorname{Sch}(X)$ for reduced, one-pointed $Z$ [cf. Proposition 1.4, (vii); [4], Proposition 1.1, (iii)], we conclude that $Y^{\log }$ is one-pointed. Thus, by applying the final portion of Proposition 1.6, (i), to $Y^{\log }$, we conclude that $Y^{\log }$ is submonic. This completes the proof of sufficiency.

Before proceeding, we review a well-known consequence of the general theory of fs $\log$ schemes.

Lemma 1.9 (Specialization morphisms associated to characteristic sheaves). Suppose that the underlying scheme $Y$ of $Y^{\log }$ is the spectrum of a strict henselian domain $A$. Write $\bar{s}$ for the tautological geometric point of $Y$ associated to the unique closed point of $Y$. Let $\bar{\eta}$ be a geometric point of $Y$ whose image in $Y$ is the unique generic point of $Y$. In the following, we shall use subscripted " $s$ ' $s$ " and " $\bar{\eta}$ 's" to denote the respective fibers at $\bar{s}, \bar{\eta}$ of sheaves on the étale site of $Y$. Then the natural "specialization morphism"

$$
P_{Y, \bar{s}} \rightarrow P_{Y, \bar{\eta}}
$$

is surjective. In particular, this specialization morphism is an isomorphism if and only if $\operatorname{rk}\left(P_{Y, \bar{s}}\right)=\operatorname{rk}\left(P_{Y, \bar{\eta}}\right)$. Finally, if $\operatorname{rk}\left(P_{Y, \bar{\eta}}\right) \geq 1$, and $a \in P_{Y, \bar{s}}$ is a sum-dominator [ $c f$. the discussion entitled "Generalities on monoids" in §0] such that, for elements $a^{*} \in M_{Y, \bar{s}}$ and $f \in A$, it holds that $a^{*} \mapsto a, a^{*} \mapsto f$, then $f=0$.

Proof. The asserted surjectivity follows immediately from the existence, étale locally, of charts that give rise to the $\log$ structure of $Y^{\log }$. If $\operatorname{rk}\left(P_{Y, \dot{s}}\right)=$ $\operatorname{rk}\left(P_{Y, \bar{\eta}}\right)$, then we thus obtain a surjection $P_{Y, \bar{s}}^{\mathrm{gp}} \rightarrow P_{Y, \bar{\eta}}^{\mathrm{gp}}$ between free abelian groups of the same rank; since such a surjection is necessarily an isomorphism, we thus conclude from the inclusion $P_{Y} \hookrightarrow P_{Y}^{\mathrm{gp}}$, that the specialization morphism $P_{Y, \bar{s}} \rightarrow P_{Y, \bar{\eta}}$ is an isomorphism, as desired. Finally, we observe that if $\operatorname{rk}\left(P_{Y, \bar{\eta}}\right) \geq 1$, and $M_{Y, \bar{s}} \ni a^{*} \mapsto f \in A$, where $a^{*}$ lifts a sum-dominator $a \in P_{Y, \bar{s}}$, then, in light of the surjectivity of the specialization morphism $P_{Y, \bar{s}} \rightarrow P_{Y, \bar{\eta}}$, it follows immediately from the discussion of sum-dominators in $\S 0$ that $a$ maps to a nonzero element $b \in P_{Y, \bar{\eta}}$. On the other hand, if we write $K$ for the quotient field of $A$, then it follows immediately from the definition of the notion of a 
$\log$ structure that the image $f \in A \subseteq K$ of any lifting $b^{*} \in M_{Y, \bar{\eta}}$ of the element $b \in P_{Y, \bar{\eta}}$ in $K$ is noninvertible, hence 0 , as desired. This completes the proof of Lemma 1.9.

Proposition 1.10 (Lower bounds on the submonic dimension). Suppose that $Y^{\log }$ is an object of $\operatorname{Sch}^{\log }\left(X^{\log }\right)$, and that $Z^{\log } \mapsto Y^{\log }$ is a monomorphism of $\operatorname{Sch}^{\log }\left(X^{\log }\right)$ such that, for suitable $n, d \in \mathbf{N}$, the log scheme $Z^{\log }$ is of rank $\boldsymbol{n}$, and the underlying scheme $Z$ of $Z^{\log }$ is of dimension $d$. Then if $n \geq 1$ (respectively, $n=0)$, then the submonic dimensions $\operatorname{dim}^{\mathrm{sm}}\left(Y^{\log }\right), \operatorname{dim}^{\mathrm{sm}}\left(Z^{\log }\right)$ of $Y^{\log }, Z^{\log }$ satisfy the inequality

$$
\begin{gathered}
\operatorname{dim}^{\mathrm{sm}}\left(Y^{\log }\right) \geq \operatorname{dim}^{\mathrm{sm}}\left(Z^{\log }\right)=d+n-1 \\
\left(\text { respectively, } \operatorname{dim}^{\mathrm{sm}}\left(Y^{\log }\right) \geq \operatorname{dim}^{\mathrm{sm}}\left(Z^{\log }\right)=d\right) .
\end{gathered}
$$

Proof. First, let us observe that it follows immediately from the definition of submonic dimension [cf. Definition 1.2, (i)] that $\operatorname{dim}^{\mathrm{sm}}\left(Y^{\log }\right) \geq \operatorname{dim}^{\mathrm{sm}}\left(Z^{\log }\right)$. In particular, we may assume without loss of generality that $Z^{\log }=Y^{\log }$. Thus, it follows immediately from Lemma 1.9 that the characteristic sheaf $P_{Y}$ is locally constant. Next, by replacing $Y^{\log }$ by the $\log$ scheme determined by a suitable subscheme of $Y$, one verifies immediately we may assume without loss of generality that the scheme $Y$ is integral. Now the case where $n=0$ is immediate [cf. Proposition 1.4, (vi)], so we may assume without loss of generality that $n \geq 1$. Thus, we may apply the theory reviewed in Lemma 1.5 to the generic point of $Y$. Moreover, one verifies immediately from the fact that $P_{Y}$ is locally constant that the objects [and properties of these objects] discussed in this theory extend to objects [and properties of these objects] over the entire scheme $Y$ [i.e., not just the generic point of $Y$ ]. In particular, by applying Lemma 1.5, (iv), (v), (vi), where we take the fs monoid " $Q$ " to be $\mathbf{N}$, we conclude that given any monomorphism $W^{\log } \mapsto Y^{\log }$, where $W^{\log }$ is a submonic object of $\operatorname{Sch}^{\log }\left(X^{\log }\right)$ whose underlying scheme $W$ is integral, there exists a monomorphism $V^{\log } \mapsto$ $Y^{\log }$, where $V^{\log }$ is a submonic object of $\operatorname{Sch}^{\log }\left(X^{\log }\right)$ whose underlying scheme $V$ is a family of $(n-1)$-dimensional tori [cf. Lemma 1.5 , (iv)] over $Y$, such that the monomorphism $W^{\log } \hookrightarrow Y^{\log }$ factors as a composite of monomorphisms $W^{\log } \longmapsto V^{\log } \longmapsto Y^{\log }$. In particular, $\operatorname{dim}(W) \leq \operatorname{dim}(V)=d+n-1$ [cf. Proposition 1.4, (vi)], so we conclude that $\operatorname{dim}^{\mathrm{sm}}\left(Y^{\log }\right)=d+n-1$, as desired.

The following generalities on log-like and scheme-like morphisms will be of use in the remainder of the present paper.

Proposition 1.11 (Generalities on log-like and scheme-like morphisms). Let $f^{\log }: Z^{\log } \rightarrow Y^{\log }$ be a morphism of $\operatorname{Sch}^{\log }\left(X^{\log }\right)$. Then:

(i) Write $U^{\log }$ for the log scheme whose underlying scheme is equal to the underlying scheme $Z$ of $Z^{\log }$ and whose log structure is the pull-back of the $\log$ structure of $Y^{\log }$ via the underlying morphism of schemes $f: Z=U \rightarrow Y$ 
associated to $f^{\log }$. Then $U^{\log }$ may be regarded, in a natural way, as an object of $\operatorname{Sch}^{\log }\left(X^{\log }\right)$, and there exists a natural factorization

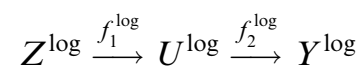

of $f^{\log }$ in $\operatorname{Sch}^{\log }\left(X^{\log }\right)$, where $f_{1}^{\log }$ is log-like, and $f_{2}^{\log }$ is scheme-like.

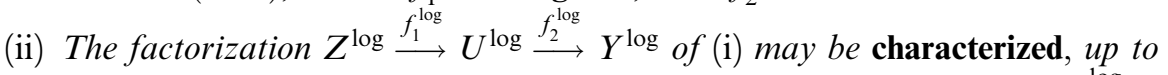
$a$ unique isomorphism, via the following universal property: The morphism $f_{2}^{\log }$ is scheme-like, and, moreover, if

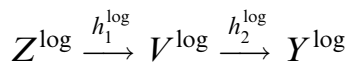

is a factorization of $f^{\log }$ in $\operatorname{Sch}^{\log }\left(X^{\log }\right)$ such that $h_{2}^{\log }$ is scheme-like, then there exists a unique scheme-like morphism $g^{\log }: U^{\log } \rightarrow V^{\log }$ such that $h_{1}^{\log }=g^{\log } \circ f_{1}^{\log }$, $h_{2}^{\log } \circ g^{\log }=f_{2}^{\log }$.

(iii) Base-change via the morphism $f_{1}^{\log }: Z^{\log } \rightarrow U^{\log }$ of (i) determines an equivalence of categories

$$
\left.\left.\operatorname{Sch}^{\log }\left(U^{\log }\right)\right|_{\text {sch-lk }} \stackrel{\sim}{\rightarrow} \operatorname{Sch}^{\log }\left(Z^{\log }\right)\right|_{\text {sch-lk }}
$$

[cf. the notational conventions of Definition 1.1, (iv)]. The morphism $f_{2}^{\log }: U^{\log } \rightarrow$ $Y^{\log }$ of (i) - which may be regarded as an object of $\left.\operatorname{Sch}^{\log }\left(Y^{\log }\right)\right|_{\text {sch-lk }}$-determines an equivalence of categories

$$
\left.\operatorname{Sch}^{\log }\left(U^{\log }\right)\right|_{\text {sch-lk }} \stackrel{\sim}{\rightarrow}\left\{\left.\operatorname{Sch}^{\log }\left(Y^{\log }\right)\right|_{\text {sch-lk }}\right\}_{f_{2}^{\log }}
$$

of $\operatorname{Sch}^{\log }\left(U^{\log }\right)$ with the category $\left\{\left.\mathrm{Sch}^{\log }\left(Y^{\log }\right)\right|_{\text {sch-lk }}\right\}_{f_{2}^{\log }}$ of objects of the category $\left.\operatorname{Sch}^{\log }\left(Y^{\log }\right)\right|_{\text {sch-lk }}$ equipped with a structure morphism to the object $f_{2}^{\log }$ of $\left.\operatorname{Sch}^{\log }\left(Y^{\log }\right)\right|_{\text {sch-lk }}$ and morphisms of the category $\left.\operatorname{Sch}^{\log }\left(Y^{\log }\right)\right|_{\text {sch-lk }}$ that are compatible with the structure morphisms to the object $f_{2}^{\log }$.

Proof. Assertions (i), (ii), and (iii) follow immediately from the various definitions involved.

\section{Section 2: The scheme structure of submonic log schemes}

In the present $\$ 2$, we give a category-theoretic reconstruction of the underlying scheme structure of submonic objects of the categories of log schemes defined in $\S 1$.

We maintain the notation of $\S 1$.

DEFINITION 2.1. Let $f^{\log }: Z^{\log } \rightarrow Y^{\log }$ be a morphism of $\operatorname{Sch}^{\log }\left(X^{\log }\right)$. Then we shall say that the morphism $f^{\log }$ is $S L E M$ [i.e., a "submonically log étale monomorphism"'] if $f^{\log }$ is a monomorphism in $\operatorname{Sch}^{\log }\left(X^{\log }\right)$, and, moreover, for any commutative diagram 


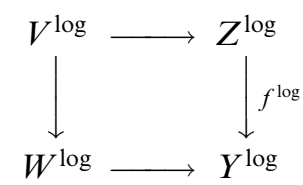

-where $V^{\log }$ and $W^{\log }$ are one-pointed and submonic, and the left-hand vertical arrow is a monomorphism in $\mathrm{Sch}^{\log }\left(X^{\log }\right)$ - of objects and morphisms in $\operatorname{Sch}^{\log }\left(X^{\log }\right)$, there exists a unique ["lifting"] morphism $W^{\log } \rightarrow Z^{\log }$ that renders the two resulting triangles in the above diagram commutative.

Proposition 2.2 (SLEM morphisms and open immersions). Let $f^{\log }: Z^{\log }$ $\rightarrow Y^{\log }$ be a morphism of $\operatorname{Sch}^{\log }\left(X^{\log }\right)$. Thus, the underlying morphism $f: Z \rightarrow$ $Y$ of $f^{\log }$ may be regarded as a morphism of $\operatorname{Sch}(X)$. Then:

(i) If $f^{\log }$ is an open immersion [cf. the discussion entitled "Log schemes" in $\S 0]$, then $f^{\log }$ is SLEM.

(ii) If $Y^{\log }$ is submonic, and $f^{\log }$ is SLEM, then $f^{\log }$ is an open immersion.

Proof. First, let us observe that any monomorphism between one-pointed objects in $\operatorname{Sch}(X)$ is necessarily a closed immersion between spectra of artinian rings [cf., e.g., the proof of [4], Corollary 1.2]. In particular, it follows from Proposition 1.4, (vi), that any monomorphism $V^{\log } \rightarrow W^{\log }$ as in Definition 2.1 is necessarily scheme-like, and, moreover, that the underlying morphism of schemes associated to any monomorphism $V^{\log } \rightarrow W^{\log }$ as in Definition 2.1 is necessarily a closed immersion between spectra of artinian rings. Thus, it is immediate that if $f^{\log }$ is an open immersion, then $f^{\log }$ is SLEM. This completes the proof of assertion (i). Now suppose that $Y^{\log }$ is submonic, and $f^{\log }$ is SLEM. Thus, it follows from Proposition 1.4, (vi), that $f^{\log }$ is scheme-like, and, moreover, that $f$ is a monomorphism in $\operatorname{Sch}(X)$. In particular, the existence of unique liftings as stipulated in Definition 2.1 implies that $f$ is an étale monomorphism in $\operatorname{Sch}(X)$, hence [cf., e.g., [4], Corollary 1.3] an open immersion. This completes the proof of assertion (ii).

Proposition 2.3 (Connectedness with respect to SLEM localizations).

(i) Let $S^{\log }$ be a connected [hence nonempty] object of $\operatorname{Sch}^{\log }\left(X^{\log }\right)[c f$. Proposition 1.3]; $U^{\log },\left\{V_{i}^{\log }\right\}_{i \in \mathbf{N}}$ nonempty objects of $\operatorname{Sch}^{\log }\left(X^{\log }\right) ; U^{\log } \hookrightarrow S^{\log }$, $\left\{V_{i}^{\log } \mapsto S^{\log }\right\}_{i \in \mathbf{N}}$ SLEM morphisms of $\operatorname{Sch}^{\log }\left(X^{\log }\right)$ such that, for each $i \in \mathbf{N}$, the morphism $V_{i}^{\log } \mapsto S^{\log }$ admits a [necessarily unique] factorization $V_{i}^{\log } \mapsto V_{i+1}^{\log }$ $\mapsto S^{\log }$ through the morphism $V_{i+1}^{\log } \mapsto S^{\log }$, and, moreover, the fiber product $U^{\log } \times{ }_{S^{\log }} V_{i}^{\log }\left[\right.$ in $\left.\operatorname{Sch}^{\log }\left(X^{\log }\right)\right]$ is empty. Then the natural map

$$
\operatorname{MinPt}\left(U^{\log }\right) \amalg\left\{\bigcup_{i \in \mathbf{N}} \operatorname{MinPt}\left(V_{i}^{\log }\right)\right\} \rightarrow \operatorname{MinPt}\left(S^{\log }\right)
$$

is injective. 
(ii) In the situation of (i), suppose further that $S^{\log }$ is submonic. Then the natural map of (i) is never surjective.

(iii) Suppose that $S^{\log }$ is a log-nodal object of $\operatorname{Sch}^{\log }\left(X^{\log }\right)$. Then, for suitable choices of $U^{\log } \mapsto S^{\log }$ and $\left\{V_{i}^{\log } \mapsto S^{\log }\right\}_{i \in \mathbf{N}}$ as in (i), the natural map of (i) is surjective.

(iv) Let $T^{\log }$ be an object of $\operatorname{Sch}^{\log }\left(X^{\log }\right)$. Then $T^{\log }$ is non-submonic if and only if there exist morphisms $U^{\log } \mapsto S^{\log }$ and $\left\{V_{i}^{\log } \mapsto S^{\log }\right\}_{i \in \mathbf{N}}$ as in (i), together with a monomorphism $S^{\log } \mapsto T^{\log }$ in $\operatorname{Sch}^{\log }\left(X^{\log }\right)$, such that the natural map of (i) is surjective.

Proof. First, we consider assertion (i). Let $i \in \mathbf{N}$. Then the injectivity of each of the natural maps $\operatorname{MinPt}\left(U^{\log }\right) \rightarrow \operatorname{MinPt}\left(S^{\log }\right), \operatorname{MinPt}\left(V_{i}^{\log }\right) \rightarrow \operatorname{MinPt}\left(S^{\log }\right)$ follows immediately from the definition of "MinPt $(-)$ ". The fact that the images of these two maps are disjoint follows immediately from the definition of "MinPt(-)", together with the assumption that the fiber product $U^{\log } \times{ }_{S^{\log }}$ $V_{i}^{\log }$ is empty. This completes the proof of assertion (i).

Next, we consider assertion (ii). Since $S^{\log }$ is submonic, it follows from Proposition 2.2, (ii), that the morphisms $U^{\log } \hookrightarrow S^{\log },\left\{V_{i}^{\log } \hookrightarrow S^{\log }\right\}_{i \in \mathbf{N}}$ are open immersions. Since [the underlying scheme of] $S^{\log }$ is connected, it thus follows from the assumption that the objects $U^{\log },\left\{V_{i}^{\log }\right\}_{i \in \mathbf{N}}$ are nonempty, whereas the fiber products $\left\{U^{\log } \times_{S^{\log }} V_{i}^{\log }\right\}_{i \in \mathbf{N}}$ are empty, that the open subscheme of $S^{\log }$ determined by the union of the images of the morphisms $U^{\log } \mapsto S^{\log }$, $\left\{V_{i}^{\log } \mapsto S^{\log }\right\}_{i \in \mathbf{N}}$ does not coincide with $S^{\log }$, and hence [cf. Proposition 1.6, (i)] that the natural map of (i) is not surjective. This completes the proof of assertion (ii).

Next, we consider assertion (iii). First, let us observe that, in light of the various assumptions imposed on $S^{\log }$, one verifies immediately that $S^{\log }$ may be regarded as the " $S$ log" that appears in Lemma 1.5, (viii). Here, the positive homomorphism $\phi_{0}: P \rightarrow J_{0}=\mathbf{N}$ of Lemma 1.5, (viii), may be taken to be the positive homomorphism " $\phi$ " of Lemma 1.5, (ii). In particular, we also obtain homomorphisms $\phi_{1}: P \rightarrow J_{1}$ and $\phi_{2}: P \rightarrow J_{2}$. Now we apply Example 0.1, where we take " $P$ " to be $P$ and " $P$ " to be $J_{2}$. This yields an infinite descending sequence

$$
P \subseteq J_{2} \subseteq \cdots \subseteq{ }^{i} P \subseteq \cdots \subseteq{ }^{1} P \subseteq{ }^{0} P
$$

-where $i \in \mathbf{N}$-of submonoids of $P^{\mathrm{gp}}$ satisfying various properties as described in Example 0.1. Suppose that, for $i \in \mathbf{N},{ }^{i} P$ is obtained as the bisecting monoid of $P$ at a positive homomorphism ' $\psi_{0}: P \rightarrow \mathbf{N}$ that is assigned the index " 2 ".

Thus, for $i \in \mathbf{N}$, the $\log$ étale monomorphism

$$
S^{\log }\left[{ }^{i} \psi_{\{0,2\}}\right] \mapsto S^{\log }
$$

of Lemma 1.5, (vii), (viii) [where we take " $\phi_{0}$ " to be ${ }^{i} \psi_{0}$ ] factors through the log étale monomorphism

$$
\left.S^{\log \left[{ }^{i+1}\right.} \psi_{\{0,2\}}\right] \mapsto S^{\log }
$$


of Lemma 1.5, (vii), (viii) [where we take " $\phi_{0}$ " to be ${ }^{i+1} \psi_{0}$ ], as well as through the log étale monomorphism

$$
S^{\log }\left[\phi_{\{0,2\}}\right] \mapsto S^{\log }
$$

of Lemma 1.5, (vii), (viii) [where we take " $\phi_{0}$ " to be $\phi_{0}$ ]. In particular, it follows from the fact that $S^{\log }\left[\phi_{\{0,1\}}\right] \times S^{\log } S^{\log }\left[\phi_{\{0,2\}}\right]=S^{\log }\left[\phi_{\{0\}}\right]$ [cf. Lemma 1.5, (vii), (viii)], together with the discussion of Example 0.1, that the fiber product $S^{\log }\left[\phi_{\{0,1\}}\right] \times S^{\log } S^{\log }\left[{ }^{i} \psi_{\{0,2\}}\right]$ is empty.

Thus, in summary, if we take $U^{\log } \neg S^{\log }$ to be the morphism

$$
S^{\log }\left[\phi_{\{0,1\}}\right] \mapsto S^{\log }
$$

and, for $i \in \mathbf{N}, V_{i}^{\log } \leadsto S^{\log }$ to be the morphism

$$
S^{\log \left[{ }^{i} \psi_{\{0,2\}}\right] \longmapsto S^{\log }}
$$

discussed above, then we obtain data as in assertion (i). Note, moreover, that it follows immediately from the discussion of Example 0.1 that the natural map of assertion (i) is surjective, as desired. This completes the proof of assertion (iii).

Finally, we observe that the sufficiency (respectively, necessity) portion of assertion (iv) follows formally from assertion (ii) (respectively, (iii)), together with Proposition 1.4, (vi) (respectively, together with Proposition 1.7, applied to $T^{\log }$ ). This completes the proof of assertion (iv).

Proposition 2.4 (Characterization of scheme-like morphisms between minimal objects). Let $h^{\log }: T^{\log } \rightarrow S^{\log }$ be a morphism between minimal objects of $\operatorname{Sch}^{\log }\left(X^{\log }\right)$. Set $r \stackrel{\text { def }}{=} \operatorname{rk}\left(S^{\log }\right) \in\{0,1\}\left[c f\right.$. Proposition 1.6, (ii)]. Then $h^{\log }$ is scheme-like if and only if there exists a connected, submonic object $Z^{\log }$ of $\operatorname{Sch}^{\log }\left(X^{\log }\right)$ such that the domain of every minimal point of $Z^{\log }$ is of rank $\boldsymbol{r}$, and, moreover, $h^{\log }$ admits a factorization

$$
T^{\log } \rightarrow Z^{\log } \rightarrow S^{\log }
$$

as the composite of a minimal point $T^{\log } \hookrightarrow Z^{\log }$ of $Z^{\log }$ with a morphism $Z^{\log } \rightarrow S^{\log }$ that admits a section $S^{\log } \rightarrow Z^{\log }$ [i.e., such that the composite $S^{\log } \rightarrow Z^{\log } \rightarrow S^{\log }$ is the identity morphism].

Proof. First, we observe that since the underlying morphism of schemes $T \rightarrow S$ necessarily arises from [i.e., by applying "Spec $(-)$ " to] a finite extension of fields, the asserted necessity follows immediately by taking $Z^{\log \stackrel{\text { def }}{=}} \mathbf{A}_{\mathbf{Z}}^{N} \times_{\mathbf{Z}} S^{\log }$ [i.e., $N$-dimensional affine space over $S^{\log }$, for a suitable positive integer $N$ ]. Here, we note that the fact that "the domain of every minimal point of this $Z^{\log }$ is of rank $r$ " follows immediately from Proposition 1.4, (vi). Thus, it remains to verify sufficiency. First, let us observe that it follows from the manifestly constructible nature of the characteristic sheaf $P_{Z}$ [cf. also Propositions 1.4, (vi); 1.6, (i), (ii)] that the assumption that "the domain of every minimal point of $Z^{\log }$ is of rank $r$ " implies that $Z^{\log }$ itself is of rank $r$, and hence [cf. Lemma 1.9] that the characteristic sheaf $P_{Z}$ is locally constant. Since the monoids 0 and $\mathbf{N}$ 
have no nontrivial automorphisms, we thus conclude that the characteristic sheaf $P_{Z}$ is constant, with fibers isomorphic to the monoid 0 (respectively, $\mathbf{N}$ ) if $r=0$ (respectively, $r=1$ ). The existence of the section $S^{\log } \rightarrow Z^{\log }$ thus implies that the morphism $Z^{\log } \rightarrow S^{\log }$ is scheme-like. Since the monomorphism $T^{\log } \mapsto Z^{\log }$ is also scheme-like [cf. Proposition 1.4, (vi)], we thus conclude that $h^{\log }$ is scheme-like, as desired. This completes the proof of sufficiency and hence of Proposition 2.4.

Proposition 2.5 (Characterization of scheme-like morphisms between sub-

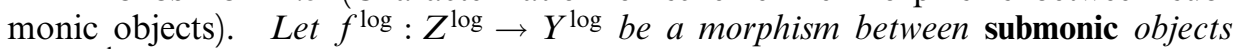
of $\operatorname{Sch}^{\log }\left(X^{\log }\right)$. Then $f^{\log }$ is scheme-like if and only if, for every minimal point $T^{\log } \hookrightarrow Z^{\log }$ of $Z^{\log }$, there exists a minimal point $S^{\log } \hookrightarrow Y^{\log }$ of $Y^{\log }$ and a schemelike morphism $T^{\log } \rightarrow S^{\log }$ of $\operatorname{Sch}^{\log }\left(X^{\log }\right)$ that fit into a commutative diagram

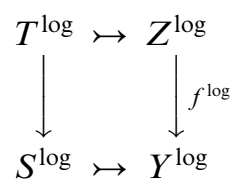

of objects of $\operatorname{Sch}^{\log }\left(X^{\log }\right)$.

Proof. The asserted necessity is immediate from the definitions and Propositions 1.4, (vi), (vii); 1.6, (ii). The asserted sufficiency follows immediately, in light of the manifestly constructible nature of the characteristic sheaves $P_{Z}, P_{Y}$, from the definitions and Propositions 1.4, (vi); 1.6, (i), (ii).

THEOREM 2.6 (Reconstruction of the scheme structure of submonic objects). For $i=1,2$, let $X_{i}^{\log }$ be a locally noetherian fs log scheme [cf. the discussion entitled "Log schemes" in §0]. For $i=1,2$, we shall write $\operatorname{Sch}^{\log }\left(X_{i}^{\log }\right)$ for the category defined at the beginning of $\$ 1$. Let

$$
\Phi: \operatorname{Sch}^{\log }\left(X_{1}^{\log }\right) \stackrel{\sim}{\rightarrow} \operatorname{Sch}^{\log }\left(X_{2}^{\log }\right)
$$

be an [arbitrary!] equivalence of categories. Then:

(i) $\Phi$ preserves the following:

(i-a) monomorphisms;

(i-b) empty objects;

(i-c) connected objects;

(i-d) minimal objects;

(i-e) minimal points;

(i-f) submonic one-pointed objects;

(i-g) ranks of minimal objects;

(i-h) SLEM morphisms;

(i-i) submonic objects;

(i-j) scheme-like morphisms between minimal objects;

(i-k) scheme-like morphisms between submonic objects;

(i-1) the submonic dimension of objects. 
(ii) For $i=1,2$, let $Y_{i}^{\log }$ be an object of $\operatorname{Sch}^{\log }\left(X_{i}^{\log }\right)$; write $Y_{i}$ for the underlying scheme of $Y_{i}^{\log }$. Suppose further that $\Phi\left(Y_{1}^{\log }\right)=Y_{2}^{\log }$. Thus, $[c f$. the portion of (i) concerning (i-i)] $Y_{1}^{\log }$ is submonic if and only if $Y_{2}^{\log }$ is. Suppose that $Y_{i}^{\log }$ is submonic for $i=1,2$. Then $\Phi$ induces an equivalence of categories

$$
\left.\left.\left(\operatorname{Sch}\left(Y_{1}\right) \stackrel{\sim}{\rightarrow}\right) \operatorname{Sch}^{\log }\left(Y_{1}^{\log }\right)\right|_{\text {sch-lk }} \stackrel{\sim}{\rightarrow} \operatorname{Sch}^{\log }\left(Y_{2}^{\log }\right)\right|_{\text {sch-lk }}\left(\stackrel{\sim}{\rightarrow} \operatorname{Sch}\left(Y_{2}\right)\right)
$$

- where the equivalences in parentheses are the natural equivalences of Definition 1.1, (iv) - that is functorial [in the evident sense!] with respect to $Y_{1}^{\log }, Y_{2}^{\log }$. Finally, the composite of the equivalences of categories in the above display induces, by applying [4], Theorem 1.7, (ii), an isomorphism of schemes

$$
Y_{1} \stackrel{\sim}{\rightarrow} Y_{2}
$$

that is functorial [in the evident sense!] with respect to $Y_{1}^{\log }, Y_{2}^{\log }$.

Proof. First, we consider assertion (i). The preservation of (i-a) is a matter of general nonsense. The preservation of (i-b) follows from Proposition 1.3, (i). The preservation of (i-c) follows from Proposition 1.3, (ii). The preservation of (i-d) and (i-e) follows immediately from the preservation of (i-a). The preservation of (i-f) follows immediately, in light of Proposition 1.8, from the preservation of (i-e). The preservation of (i-g) follows immediately, in light of Proposition 1.6, (iii), from the preservation of (i-d). The preservation of $(\mathrm{i}-\mathrm{h})$ follows immediately from the preservation of (i-a) and (i-f). The preservation of (i-i) follows immediately, in light of Proposition 2.3, (iv), from the preservation of (i-a), (i-b), (i-c), (i-e), and (i-h). The preservation of (i-j) follows immediately, in light of Proposition 2.4, from the preservation of (i-c), (i-d), (i-e), (i-g), and (i-i). The preservation of (i-k) follows immediately, in light of Proposition 2.5, from the preservation of (i-e), (i-i), and (i-j). This completes the proof of assertion (i), except for the verification of the preservation of (i-1). Assertion (ii) follows immediately [i.e., in the spirit of [4], Corollary 2.15] from the portion of assertion (i) concerning the preservation of (i-k). Here, we note that the functoriality of the isomorphism of schemes in the final display in the statement of assertion (ii) follows immediately from the characterization given in Proposition 1.11, (ii), of the factorization discussed in Proposition 1.11, (i), together with the natural equivalences of categories discussed in Proposition 1.11, (iii). Finally, the portion of assertion (i) concerning the preservation of (i-1) follows from the portion of assertion (i) concerning the preservation of (i-a), (i-i), together with the isomorphisms of schemes obtained in assertion (ii).

LEMMA 2.7 (Characterization of isomorphisms among positive homomorphisms). Let $\xi: P \rightarrow Q$ be a positive homomorphism between fs monoids such that $\operatorname{rk}(P) \geq \operatorname{rk}(Q)$, and, moreover, the following condition is satisfied:

Every positive homomorphism $\phi: P \rightarrow \mathbf{N}$ admits a factorization $P \rightarrow Q \rightarrow$ $\mathbf{N}$ as a composite of $\xi$ with a positive homomorphism $\psi: Q \rightarrow \mathbf{N}$.

Then $\xi$ is an isomorphism. 
Proof. First, let us observe that, by Lemma 1.5, (ii), there exists a positive homomorphism $\phi^{\dagger}: P \rightarrow \mathbf{N}$. Next, let us observe that if $p$ is a prime number, then given a surjective homomorphism $\bar{\zeta}: P^{\mathrm{gp}} \rightarrow \mathbf{F}_{p}$, there exists a homomorphism $\zeta: P^{\mathrm{gp}} \rightarrow \mathbf{Z}$ whose composite with the natural surjection $\mathbf{Z} \rightarrow \mathbf{F}_{p}$ is equal to $\bar{\zeta}$. [Indeed, this follows immediately from the fact that $P^{\mathrm{gp}}$ is a finitely generated free abelian group - cf. the discussion entitled "Generalities on monoids" in §0.] In particular, it follows from the fact that $P$ is a finitely generated monoid that, for sufficiently large $n \in \mathbf{N}$, the homomorphism $\left(\zeta+p^{n} \cdot\left(\phi^{\dagger}\right)^{\mathrm{gp}}\right)$ : $P^{\mathrm{gp}} \rightarrow \mathbf{Z}$ coincides with $\bar{\zeta}$ when composed with the natural surjection $\mathbf{Z} \rightarrow \mathbf{F}_{p}$ and, moreover, determines a positive homomorphism $\phi: P \rightarrow \mathbf{N}$. In particular, it follows from the hypotheses imposed on $\xi$ that $\phi$ admits a factorization $P \rightarrow Q \rightarrow \mathbf{N}$ as a composite of $\xi$ with a positive homomorphism $\psi: Q \rightarrow \mathbf{N}$. Since the resulting composite $P^{\mathrm{gp}} \rightarrow Q^{\mathrm{gp}} \rightarrow \mathbf{Z} \rightarrow \mathbf{F}_{p}$ coincides with $\bar{\zeta}$, we thus conclude, by allowing $p$ and $\bar{\zeta}$ to vary, that the reduction of the homomorphism of finitely generated free abelian groups $\xi^{\mathrm{gp}}: P^{\mathrm{gp}} \rightarrow Q^{\mathrm{gp}}$ modulo any prime number is injective, and, hence, since $\operatorname{rk}(P) \geq \operatorname{rk}(Q)$, that $\xi^{\mathrm{gp}}: P^{\mathrm{gp}} \rightarrow Q^{\mathrm{gp}}$ is an isomorphism. That is to say, $P$ and $Q$ may be regarded as finitely generated saturated monoids within a single $\mathbf{Z}$-module $P^{\mathrm{gp}} \stackrel{\sim}{\rightarrow} Q^{\mathrm{gp}}$. In particular, it follows from well-known properties of fs monoids [cf., e.g., [4], Lemma 2.5, (iv)] that the hypotheses imposed on $\xi$ imply that $\xi$ is an isomorphism, as desired.

Proposition 2.8 (Characterization of scheme-like morphisms between re-

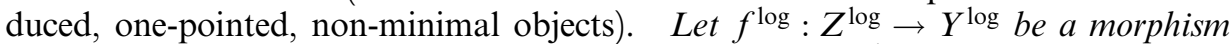
between reduced, one-pointed, non-minimal objects of $\operatorname{Sch}^{\log }\left(X^{\log }\right)$. Then $f^{\log }$ is scheme-like if and only if $\operatorname{dim}^{\mathrm{sm}}\left(Z^{\log }\right) \leq \operatorname{dim}^{\mathrm{sm}}\left(Y^{\log }\right)$, and, moreover, the following condition is satisfied:

Let $S^{\log }$ be a minimal object of $\operatorname{Sch}^{\log }\left(X^{\log }\right), h^{\log }: S^{\log } \rightarrow Y^{\log }$ a morphism of $\mathrm{Sch}^{\log }\left(X^{\log }\right)$. Then there exists a commutative diagram of morphisms of $\mathrm{Sch}^{\log }\left(X^{\log }\right)$

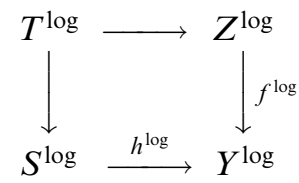

in which the left-hand vertical arrow $T^{\log } \rightarrow S^{\log }$ is a scheme-like morphism between minimal objects of $\operatorname{Sch}^{\log }\left(X^{\log }\right)$.

Proof. First of all, we observe that the asserted necessity follows immediately from Proposition 1.10, together with the definition of the term "schemelike". Thus, it suffices to verify the sufficiency of the condition that appears in the statement of Proposition 2.8. To this end, let us first observe that it follows [cf. Proposition 1.6, (ii)] from the assumption that $Z^{\log }$ and $Y^{\log }$ are nonminimal that $\operatorname{rk}\left(Z^{\log }\right) \geq 2, \operatorname{rk}\left(Y^{\log }\right) \geq 2$. Thus, it follows from Proposition 1.10 
that $\operatorname{rk}\left(Z^{\log }\right)=\operatorname{dim}^{\mathrm{sm}}\left(Z^{\log }\right)+1 \leq \operatorname{dim}^{\mathrm{sm}}\left(Y^{\log }\right)+1=\operatorname{rk}\left(Y^{\log }\right)$. Next, let us observe-i.e., by applying Lemma 1.5, (v), as in the proof of Proposition 1.6, (i)that the condition under consideration implies that the restriction to a geometric point of $Z^{\log }$ of the morphism of characteristic sheaves $\left.P_{Y}\right|_{Z} \rightarrow P_{Z}$ induced by $f^{\log }$ satisfies the condition discussed in Lemma 2.7. In particular, we conclude from Lemma 2.7 that this morphism $\left.P_{Y}\right|_{Z} \rightarrow P_{Z}$ is, in fact, an isomorphism, and hence that $f^{\log }$ is scheme-like, as desired.

Definition 2.9. (i) Let $Z$ be a scheme. Then we shall refer to a point $z$ of the underlying topological space of $Z$ as a locally closed point if $z$ determines a closed point of some open subscheme of $Z$. Write

\section{$\operatorname{LCPt}(Z)$}

for the set of locally closed points of $Z$.

(ii) Let $Z^{\log }$ be an object of $\operatorname{Sch}^{\log }\left(X^{\log }\right)$. For $i=1,2$, let $U_{i}^{\log }$ be a minimal object of $\operatorname{Sch}^{\log }\left(X^{\log }\right)$ and $f_{i}^{\log }: U_{i}^{\log } \rightarrow Z^{\log }$ an arrow of $\operatorname{Sch}^{\log }\left(X^{\log }\right)$. Then we shall say that $f_{1}^{\log }$ and $f_{2}^{\log }$ are point-equivalent if there exist a morphism $f_{W}^{\log }: W^{\log } \rightarrow Z^{\log }$ and, for each $i=1,2$, a morphism $h_{i}^{\log }: V_{i}^{\log } \rightarrow U_{i}^{\log }$ between minimal objects of $\mathrm{Sch}^{\log }\left(X^{\log }\right)$ such that $W^{\log }$ is $\log$-nodal, and, moreover, for each $i=1,2$, the composite morphism $f_{i}^{\log } \circ h_{i}^{\log }: V_{i}^{\log } \rightarrow Z^{\log }$ admits a factorization $V_{i}^{\log } \rightarrow W^{\log } \rightarrow Z^{\log }$ through $f_{W}^{\log }: W^{\log } \rightarrow Z^{\log }$.

(iii) Let $Z^{\log }$ be an object of $\operatorname{Sch}^{\log }\left(X^{\log }\right)$ whose underlying scheme we denote by $Z, z \in \operatorname{LCPt}\left(Z^{\log }\right) \stackrel{\text { def }}{=} \operatorname{LCPt}(Z)$. Then a monomorphism $H^{\log } \mapsto Z^{\log }$ in $\operatorname{Sch}^{\log }\left(X^{\log }\right)$ will be called a point-hull at $z$ if $H^{\log }$ is one-pointed, and, moreover, every morphism $S^{\log } \rightarrow Z^{\log }$ in $\operatorname{Sch}^{\log }\left(X^{\log }\right)$ from a minimal object $S^{\log }$ to $Z^{\log }$ that maps the unique point of the underlying scheme $S$ of $S^{\log }$ to $z$ factors [necessarily uniquely!] through the given monomorphism $H^{\log } \hookrightarrow Z^{\log }$. A pointhull $H^{\log } \mapsto Z^{\log }$ at $z$ will be called a minimal point-hull at $z$ if every monomorphism $H_{1}^{\log } \hookrightarrow H^{\log }$ in $\operatorname{Sch}^{\log }\left(X^{\log }\right)$ for which the composite $H_{1}^{\log } \hookrightarrow H^{\log } \hookrightarrow$ $Z^{\log }$ is a point-hull at $z$ is necessarily an isomorphism. An arrow of $\operatorname{Sch}^{\log }\left(X^{\log }\right)$ which is a minimal point-hull at some element of $\operatorname{LCPt}(-)$ of the codomain of the arrow will be referred to as a minimal point-hull. Thus, if $Z^{\log }$ is one-pointed, and one restricts one's attention to monomorphisms with one-pointed domains, then the notion of a point-hull (respectively, minimal point-hull) at $z$ is identical to the notion of a hull (respectively, minimal hull) [cf. Definition 1.1, (iii)].

Proposition 2.10 (Point-classes and minimal point-hulls). Let $Z^{\log }$ be an object of $\operatorname{Sch}^{\log }\left(X^{\log }\right)$. For $i=1,2$, let $U_{i}^{\log }$ be a minimal object of $\operatorname{Sch}^{\log }\left(X^{\log }\right)$ and $f_{i}^{\log }: U_{i}^{\log } \rightarrow Z^{\log }$ an arrow of $\operatorname{Sch}^{\log }\left(X^{\log }\right)$. For $i=1,2$, write $Z, U_{i}$ for the underlying schemes of $Z^{\log }, U_{i}^{\log }$, respectively. Then:

(i) $Z^{\log }$ is one-pointed if and only if the set $\operatorname{LCPt}\left(Z^{\log }\right)=\operatorname{LCPt}(Z)$ is of cardinality one.

(ii) Let $z$ be a point of the underlying topological space of $Z$. Then the following conditions are equivalent: (ii-a) $z$ is locally closed; (ii-b) $z$ appears as 
the image of a morphism $U \rightarrow Z$ of $\operatorname{Sch}(X)$ for some minimal object $U$ [cf. [4], Proposition 1.1, (ii)] of $\mathrm{Sch}(X)$; (ii-c) $z$ appears as the image of a morphism $U^{\log } \rightarrow Z^{\log }$ of $\operatorname{Sch}^{\log }\left(X^{\log }\right)$ for some minimal object $U^{\log }$ of $\operatorname{Sch}^{\log }\left(X^{\log }\right)$.

(iii) Write $z_{i}$ for the image in $Z$ via [the underlying morphism of schemes associated to $f_{i}^{\log }$ of the unique point of $U_{i}$. Then the arrows $f_{1}^{\log }$ and $f_{2}^{\log }$ are point-equivalent if and only if $z_{1}=z_{2}$. In particular, the notion of pointequivalence determines an equivalence relation on the collection [i.e., which, strictly speaking, is not necessarily a set!] of arrows in $\operatorname{Sch}^{\log }\left(X^{\log }\right)$ from minimal objects of $\operatorname{Sch}^{\log }\left(X^{\log }\right)$ to $Z^{\log }$. Write

$$
\operatorname{PtCl}\left(Z^{\log }\right)
$$

for the set of equivalence classes of such arrows. We shall refer to an element of $\operatorname{PtCl}\left(Z^{\log }\right)$ as a point-class of $Z^{\log }$.

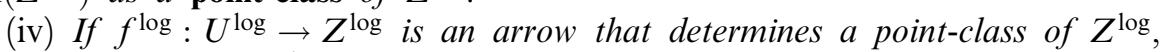
then let us write $\operatorname{Im}\left(f^{\log }\right)$ for the image in $Z$ via [the underlying morphism of schemes associated to $f^{\log }$ of the unique point of the underlying scheme $U$ of $U^{\log }$. Then the assignment $f^{\log } \mapsto \operatorname{Im}\left(f^{\log }\right)$ determines a bijection of sets

$$
\operatorname{PtCl}\left(Z^{\log }\right) \stackrel{\sim}{\rightarrow} \operatorname{LCPt}\left(Z^{\log }\right)=\operatorname{LCPt}(Z)
$$

that is functorial [in the evident sense] with respect to $Z^{\log }$.

(v) Let $z \in \operatorname{LCPt}(Z)$. Write $z^{\log }$ for the reduced, one-pointed object of $\operatorname{Sch}^{\log }\left(X^{\log }\right)$ obtained by restricting the log structure of $Z^{\log }$ to $z$. Then $a$

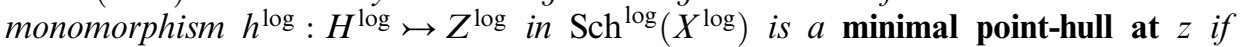
and only if $h^{\log }$ induces an isomorphism $H^{\log \stackrel{\sim}{\rightarrow}} z^{\log }$.

Proof. First, we observe that assertion (i) follows immediately from the various definitions involved [cf. also [4], Proposition 1.1, (i)]. Next, we consider assertion (ii). First, we recall from [4], Proposition 1.1, (ii), that an object of $\operatorname{Sch}(X)$ is minimal if and only if it is reduced and one-pointed. Next, we recall from Proposition 1.6, (ii), that a minimal object of $\operatorname{Sch}^{\log }\left(X^{\log }\right)$ is necessarily reduced and one-pointed. Now the implication (ii-a) $\Rightarrow$ (ii-b) follows immediately. In a similar vein, the implication (ii-a) $\Rightarrow$ (ii-c) follows immediately, by applying Proposition 1.6, (i). To verify the implications (ii-b) $\Rightarrow$ (ii-a), (ii-c) $\Rightarrow$ (ii-a), it suffices to verify that if $U$ is a one-pointed object of $\operatorname{Sch}(X)$, then the image via any morphism $U \rightarrow Z$ of $\operatorname{Sch}(X)$ of the unique point of $U$ is a locally closed point of $Z$. Note that, by considering the schematic closure of such a morphism in a suitable affine open of $Z$, we may assume without loss of generality that $U$ and $Z$ are affine, and that the morphism [of finite type!] $U \rightarrow Z$ has dense image. Since this image [which consists of a single point!] is necessarily constructible, hence contains a dense open subset of the underlying topological space of $Z$, we thus conclude that we may assume, after replacing $Z$ by a suitable affine open of $Z$, that the morphism $U \rightarrow Z$ is surjective, i.e., that $Z$ is one-pointed. This completes the proof of assertion (ii). 
Next, we consider assertion (iii). Since minimal objects of $\operatorname{Sch}^{\log }\left(X^{\log }\right)$ are necessarily one-pointed [cf. Proposition 1.6, (ii)], the necessity portion of the asserted equivalence follows immediately from the various definitions involved. Thus, it suffices to verify the sufficiency portion of the asserted equivalence. To this end, let us first observe that, since minimal objects of $\operatorname{Sch}^{\log }\left(X^{\log }\right)$ are necessarily reduced [cf. Proposition 1.6, (ii)], we may assume without loss of generality that $Z^{\log }$ is reduced and one-pointed. Also, by base-changing to a suitable finite extension of the field whose spectrum is $Z$, we conclude that we may assume without loss of generality that $f_{1}^{\log }$ and $f_{2}^{\log }$ are log-like, and that $Z^{\log }$ is split. Thus, by considering a suitable splitting as in Lemma 1.5, (i), one verifies immediately that, to complete the proof of sufficiency, it suffices to verify the following assertion concerning $f_{s}$ monoids:

Let $P$ be an $f_{s}$ monoid. For $i=1,2$, let $\phi_{i}: P \rightarrow \mathbf{N}$ be a positive homomorphism of fs monoids. Then there exist an $f s$ monoid $Q$ of rank two and a positive homomorphism $\psi: P \rightarrow Q$ of fs monoids such that, for $i=1,2$, the homomorphism $2 \cdot \phi_{i}: P \rightarrow \mathbf{N}$ [i.e., the composite of $\phi_{i}$ with the positive homomorphism $\mathbf{N} \rightarrow \mathbf{N}$ given by multiplication by 2] admits a factorization $P \rightarrow Q \rightarrow \mathbf{N}$ as the composite of $\psi$ with some positive homomorphism $\psi_{i}: Q \rightarrow \mathbf{N}$.

This assertion concerning fs monoids may be verified as follows. For $i=1,2$, write $N_{i}^{\mathrm{gp}} \subseteq P^{\mathrm{gp}}$ for the kernel of the morphism $\phi_{i}^{\mathrm{gp}}: P^{\mathrm{gp}} \rightarrow \mathbf{Z}$. If $N_{1}^{\mathrm{gp}}=N_{2}^{\mathrm{gp}}$, then one verifies immediately that one obtains data as desired by considering the factorization $\mathbf{N} \rightarrow \mathbf{N} \oplus \mathbf{N} \rightarrow \mathbf{N}$ [i.e., determined by the assignments $\mathbf{N} \ni 1 \mapsto$ $(1,1) \in \mathbf{N} \oplus \mathbf{N}$ and $\mathbf{N} \oplus \mathbf{N} \ni(a, b) \mapsto a+b \in \mathbf{N}]$ of the homomorphism $\mathbf{N} \rightarrow \mathbf{N}$ given by multiplication by 2 . Thus, we may assume without loss of generality that $N_{1}^{\mathrm{gp}} \neq N_{2}^{\mathrm{gp}}$. Write $Q$ for the saturation [cf. [4], Lemma 2.5, (ii)] of the image of $P$ in $\left(P^{\mathrm{gp}} / N_{1}^{\mathrm{gp}}\right) \oplus\left(P^{\mathrm{gp}} / N_{2}^{\mathrm{gp}}\right)(\cong \mathbf{Z} \oplus \mathbf{Z})$. Thus, we obtain a natural positive homomorphism of monoids $\psi: P \rightarrow Q$ such that, for $i=1,2, \phi_{i}: P \rightarrow \mathbf{N}$ admits a factorization $P \rightarrow Q \rightarrow \mathbf{N}$ as the composite of $\psi$ with some positive homomorphism $\psi_{i}: Q \rightarrow \mathbf{N}$. Here, we note that the positivity of $\psi_{i}$ follows immediately from the positivity of $\phi_{i}$. Also, we observe that the positivity of $\psi_{i}$ implies that the monoid $Q$ has no nonzero invertible elements. We thus conclude that $Q$ is an $f_{S}$ monoid of rank two, as desired. This completes the proof of assertion (iii). Assertion (iv) follows immediately from assertion (iii), together with the equivalence (ii-a) $\Leftrightarrow$ (ii-c) of assertion (ii).

Finally, we consider assertion (v). First, we consider the sufficiency portion of the asserted equivalence. To verify this sufficiency, it suffices to verify that the natural monomorphism $h_{z}^{\log }: z^{\log } \hookrightarrow Z^{\log }$ [cf. Proposition 1.4, (vii)] is a minimal point-hull at $z$. The fact that $h_{z}^{\log }$ is a point-hull at $z$ follows immediately from the various definitions involved. Now suppose that $h_{1}^{\log }: H_{1}^{\log } \nrightarrow z^{\log }$ is a mono-

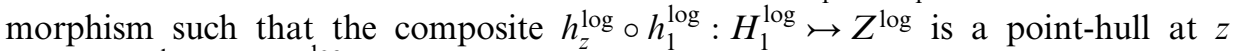
[so both $z^{\log }$ and $H_{1}^{\log }$ are one-pointed]. Then one verifies immediately that, by applying Lemma 1.5, (v), as in the proof of Proposition 1.6, (i), it follows from 
Proposition 1.4, (iii), and Lemma 2.7 that $h_{1}^{\log }$ is scheme-like, and hence, by Proposition 1.4, (v); [4], Proposition 1.1, (ii), that $h_{1}^{\log }$ is an isomorphism, as desired. Thus, to complete the proof of assertion (v), it suffices to verify the necessity portion of the asserted equivalence. First, let us observe that it follows from the existence of the natural monomorphism $H_{\mathrm{red}}^{\log } \neg H^{\log }$, together with the definition of the notion of a minimal point-hull, that $H^{\log }$ is reduced and one-pointed. Thus, it follows immediately from Proposition 1.6, (i), that $h^{\log }$ induces a monomorphism $H^{\log } \longmapsto z^{\log }$. Since we have already verified that $h_{z}^{\log }$ is a minimal point-hull at $z$, we thus conclude that this monomorphism $H^{\log } \mapsto z^{\log }$ is an isomorphism, as desired. This completes the proof of assertion (v).

Proposition 2.11 (Characterization of scheme-like morphisms between arbitrary objects). Let $f^{\log }: Z^{\log } \rightarrow Y^{\log }$ be a morphism between arbitrary objects of $\operatorname{Sch}^{\log }\left(X^{\log }\right)$. Then $f^{\log }$ is scheme-like if and only if, for every minimal pointhull $h^{\log }: T^{\log } \rightarrow Z^{\log }$, there exists a commutative diagram of morphisms of $\operatorname{Sch}^{\log }\left(X^{\log }\right)$

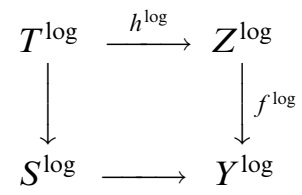

in which the lower horizontal arrow $S^{\log } \rightarrow Y^{\log }$ is a minimal point-hull, and the left-hand vertical arrow $T^{\log } \rightarrow S^{\log }$ is a scheme-like morphism between reduced, one-pointed objects of $\operatorname{Sch}^{\log }\left(X^{\log }\right)$.

Proof. The asserted equivalence follows immediately, in light of the manifestly constructible nature of the characteristic sheaves $P_{Z}, P_{Y}$, from Proposition 2.10 , (v), together with the definition of the term "scheme-like".

COROLlary 2.12 (Conditional reconstruction of the scheme structure of arbitrary objects). Suppose that we are in the situation of Theorem 2.6, and that $\Phi$ satisfies the following condition:

$\left(*_{\text {nod }}\right)$ an object of $\operatorname{Sch}^{\log }\left(X_{1}^{\log }\right)$ is log-nodal if and only if its image via $\Phi$ is a log-nodal object of $\operatorname{Sch}^{\log }\left(X_{2}^{\log }\right)$.

Then:

(i) $\Phi$ preserves the following:

(i-a) point-equivalent pairs of arrows;

(i-b) the set-valued functor $\operatorname{LCPt}(-)$ [up to natural equivalence];

(i-c) arrows which are minimal point-hulls;

(i-d) scheme-like morphisms between arbitrary objects. 
(ii) For $i=1,2$, let $Y_{i}^{\log }$ be an object of $\operatorname{Sch}^{\log }\left(X_{i}^{\log }\right)$; write $Y_{i}$ for the underlying scheme of $Y_{i}^{\log }$. Suppose further that $\Phi\left(Y_{1}^{\log }\right)=Y_{2}^{\log }$. Then $\Phi$ induces an equivalence of categories

$$
\left.\left.\left(\operatorname{Sch}\left(Y_{1}\right) \stackrel{\sim}{\rightarrow}\right) \operatorname{Sch}^{\log }\left(Y_{1}^{\log }\right)\right|_{\text {sch-lk }} \stackrel{\sim}{\rightarrow} \operatorname{Sch}^{\log }\left(Y_{2}^{\log }\right)\right|_{\text {sch-lk }}\left(\stackrel{\sim}{\rightarrow} \operatorname{Sch}\left(Y_{2}\right)\right)
$$

- where the equivalences in parentheses are the natural equivalences of Definition 1.1, (iv) - that is functorial [in the evident sense!] with respect to $Y_{1}^{\log }, Y_{2}^{\log }$. Finally, the composite of the equivalences of categories in the above display induces, by applying [4], Theorem 1.7, (ii), an isomorphism of schemes

$$
Y_{1} \stackrel{\sim}{\rightarrow} Y_{2}
$$

that is functorial [in the evident sense!] with respect to $Y_{1}^{\log }, Y_{2}^{\log }$.

Proof. First, we consider assertion (i). The preservation of (i-a) follows immediately, in light of the preservation of (i-d) asserted in Theorem 2.6, (i), from the condition $\left(*_{\text {nod }}\right)$, together with the definition of the term "point-equivalent". The prservation of (i-b) now follows from the preservation of (i-a), together with the bijection of Proposition 2.10, (iv). The preservation of (i-c) then follows from the preservation of (i-b) [cf. also the preservation of (i-a), (i-d) asserted in Theorem 2.6, (i)], together with the equivalence of Proposition 2.10, (i). The preservation of (i-d) follows, in light of the preservation of (i-c), from Propositions $2.8 ; 2.10,(\mathrm{v}) ; 2.11$ [cf. also the preservation of $(\mathrm{i}-\mathrm{d}),(\mathrm{i}-\mathrm{j}),(\mathrm{i}-\mathrm{l})$ asserted in Theorem 2.6, (i)]. This completes the proof of assertion (i). Now assertion (ii) follows immediately [i.e., in the spirit of Theorem 2.6, (ii); [4], Corollary 2.15] from the portion of assertion (i) concerning the preservation of (i-d). Here, we note that the functoriality of the isomorphism of schemes in the final display in the statement of assertion (ii) follows immediately from the characterization given in Proposition 1.11, (ii), of the factorization discussed in Proposition 1.11, (i), together with the natural equivalences of categories discussed in Proposition 1.11, (iii).

\section{Section 3: Seamless partitions of orientable log schemes}

In the present $\S 3$, we discuss the notion of a seamless partition of an orientable $\log$ scheme. This notion leads naturally to a category-theoretic characterization of log-nodal objects, which we apply to eliminate the dependence on the condition " $\left(*_{\text {nod }}\right)$ " in Corollary 2.12 .

We maintain the notation of $\S 2$.

Definition 3.1. (i) Suppose that $Y^{\log }$ is an object of $\operatorname{Sch}^{\log }\left(X^{\log }\right)$. Then we shall say that $Y^{\log }$ is $\log$-Dedekind if it satisfies the following conditions:

(i-a) $\operatorname{dim}^{\mathrm{sm}}\left(Y^{\log }\right) \leq 1$;

(i-b) if $Z^{\log }$ is a minimal object of $\operatorname{Sch}^{\log }\left(X^{\log }\right)$ such that there exists a morphism $Z^{\log } \rightarrow Y^{\log }$ in $\operatorname{Sch}^{\log }\left(X^{\log }\right)$, then $Z^{\log }$ is of rank one; 
(i-c) if $Z^{\log }$ is a nonempty submonic object of $\operatorname{Sch}^{\log }\left(X^{\log }\right)$, with underlying scheme $Z$, such that there exists a SLEM morphism $Z^{\log } \mapsto Y^{\log }$ in $\operatorname{Sch}^{\log }\left(X^{\log }\right)$, then the closed subscheme $Z_{\text {red }} \subseteq Z$ is regular and of positive dimension.

If $y$ is a point of the underlying scheme $Y$ of a log-Dedekind object $Y^{\log }$, and the fiber of $P_{Y}$ at some geometric point of $Y$ that maps to $y$ is of rank two, then we shall say that $y$ is a nodal point of $Y^{\log }$.

(ii) Suppose that $Y^{\log }$ is a $\log$-Dedekind object of $\operatorname{Sch}^{\log }\left(X^{\log }\right)$. For $i=1,2$, let $Z_{i}^{\log }$ be a connected [hence nonempty], submonic object of $\operatorname{Sch}^{\log }\left(X^{\log }\right)$ and

$$
f_{i}^{\log }: Z_{i}^{\log } \mapsto Y^{\log }
$$

a SLEM morphism. We shall say that $f_{1}^{\log }$ and $f_{2}^{\log }$ are submonically equivalent if the fiber product $Z_{12}^{\log \stackrel{\text { def }}{=}} Z_{1}^{\log } \times_{Y^{\log }} Z_{2}^{\log }$ determined by $f_{1}^{\log }$ and $f_{2}^{\log }$ is nonempty. [Here, we note that, for $i=1,2$, the projection $Z_{12}^{\log } \rightarrow Z_{i}^{\log }$, is SLEM, hence, by Proposition 2.2, (ii), an open immersion, whose image is, by condition (i-c), dense whenever it is nonempty.] One verifies immediately that the notion of submonic equivalence determines an equivalence relation on the collection [i.e., which, strictly speaking, is not necessarily a set!] of arrows of $\operatorname{Sch}^{\log }\left(X^{\log }\right)$ which are SLEM morphisms from connected, submonic objects of $\operatorname{Sch}^{\log }\left(X^{\log }\right)$ to $Y^{\log }$. Write

$$
\operatorname{SmCp}\left(Y^{\log }\right)
$$

for the set of equivalence classes of such arrows. We shall refer to an element of $\operatorname{SmCp}\left(Y^{\log }\right)$ as a submonic component of $Y^{\log }$.

(iii) Suppose that $Y^{\log }$ is a log-Dedekind object of $\operatorname{Sch}^{\log }\left(X^{\log }\right)$. If $h^{\log }$ : $H^{\log } \longmapsto Y^{\log }$ is a monomorphism of $\operatorname{Sch}^{\log }\left(X^{\log }\right)$, then we shall write

$$
\mathrm{Chn}\left(h^{\log }\right) \subseteq \operatorname{SmCp}\left(Y^{\log }\right)
$$

for the subset of submonic components for which there exists a representative arrow $Z^{\log } \mapsto Y^{\log }$ that admits a factorization $Z^{\log } \longmapsto H^{\log } \hookrightarrow Y^{\log }$ through $h^{\log }$ : $H^{\log } \mapsto Y^{\log }$. If $C \subseteq \operatorname{SmCp}\left(Y^{\log }\right)$ is a nonempty subset, then we shall refer to $C$ as a chain if there exists a SLEM morphism $h^{\log }: H^{\log } \neg Y^{\log }$ of $\operatorname{Sch}^{\log }\left(X^{\log }\right)$ such that $H^{\log }$ is connected [hence nonempty!], and $C=\operatorname{Chn}\left(h^{\log }\right)$. If $C \subseteq$ $\operatorname{SmCp}\left(Y^{\log }\right)$ is a subset, then we shall refer to $C$ as an $\mathbf{N}$-chain if there exists a collection $\left\{C_{i}\right\}_{i \in \mathbf{N}}$ of chains $C_{i} \subseteq \operatorname{SmCp}\left(Y^{\log }\right)$ such that $C=\bigcup_{i \in \mathbf{N}} C_{i}$, and $C_{i} \subseteq C_{i+1}$ for all $i \in \mathbf{N}$.

Proposition 3.2 (First properties of log-Dedekind objects). Suppose that $Y^{\log }$ is a log-Dedekind object of $\operatorname{Sch}^{\log }\left(X^{\log }\right)$. Then:

(i) $Y^{\log }$ is of rank $\leq \mathbf{2}$.

(ii) The non-nodal points of the underlying scheme $Y$ of $Y^{\log }$ form an open subset of the underlying topological space of $Y$. Write $Y_{\mathrm{sm}} \subseteq Y$ for the corre- 
sponding open subscheme and $Y_{\mathrm{sm}}^{\mathrm{log}}$ for the log scheme obtained by restricting the log structure of $Y^{\log }$ to $Y_{\mathrm{sm}}$. Then the complement of $Y_{\mathrm{sm}}$ in $Y$ is a closed subscheme of $Y$ of dimension zero, and $Y_{\mathrm{sm}}^{\log }$ is submonic. We shall refer to $Y_{\mathrm{sm}}^{\log }$ as the submonic locus of $Y^{\log }$.

(iii) Let $Z^{\log }$ be a nonempty submonic object of $\operatorname{Sch}^{\log }\left(X^{\log }\right)$ and $Z^{\log } \mapsto Y^{\log }$ a SLEM morphism. Then the closed subscheme $Z_{\mathrm{red}} \subseteq Z$ of the underlying scheme $Z$ of $Z^{\log }$ is regular and of dimension one, and $Z^{\log }$ is of rank one. In particular, [cf. Proposition 2.2, (i)] $\left(Y_{\mathrm{sm}}\right)_{\mathrm{red}}$ is regular and of dimension one, and $Y_{\mathrm{sm}}^{\log }$ is of rank one.

(iv) Let $f^{\log }: Z^{\log } \mapsto Y^{\log }$ be a SLEM morphism from a connected, submonic object $Z^{\log }$ of $\mathrm{Sch}^{\log }\left(X^{\log }\right)$ to $Y^{\log }$. Then $f^{\log }$ either admits a factorization $Z^{\log } \mapsto Y_{\mathrm{sm}}^{\log } \mapsto Y^{\log }$ as the composite of an open immersion $Z^{\log } \mapsto Y_{\mathrm{sm}}^{\log }$ with the natural monomorphism $Y_{\mathrm{sm}}^{\log } \nrightarrow Y^{\log }$ or maps the entire underlying scheme $Z$ of $Z^{\log }$ to some nodal point $y$ of $Y^{\log }$. In the former case, we shall say that $f^{\log }$ is non-nodal; in the latter case, we shall say that $f^{\log }$ is nodal and lies over $y$. We shall also apply this terminology "non-nodal"/"nodal" to the element of $\operatorname{SmCp}\left(Y^{\log }\right)$ determined by $f^{\log }$.

(v) Let $y$ be a nodal point of $Y^{\log }$. Then the subset

$$
\operatorname{SmCp}\left(Y^{\log }\right)_{y} \subseteq \operatorname{SmCp}\left(Y^{\log }\right)
$$

of nodal elements that lie over y forms an $\mathbf{N}$-chain. Moreover, every morphism $H^{\log } \rightarrow Y^{\log }$ in $\operatorname{Sch}^{\log }\left(X^{\log }\right)$ from a minimal object $H^{\log }$ to $Y^{\log }$ that maps the unique point of the underlying scheme of $H^{\log }$ to $y$ factors through some representative of an element of $\operatorname{SmCp}\left(Y^{\log }\right)_{y}$.

(vi) Every element $\gamma \in \operatorname{SmCp}\left(Y^{\log }\right)$ admits a "maximal" representative arrow $f^{\log }: Z^{\log } \mapsto Y^{\log }$, i.e., a representative arrow such that every arrow $U^{\log } \mapsto Y^{\log }$ of $\operatorname{Sch}^{\log }\left(X^{\log }\right)$ that is submonically equivalent to $f^{\log }$ admits a factorization

$$
U^{\log } \mapsto Z^{\log } \mapsto Y^{\log }
$$

as the composite of some open immersion $U^{\log } \hookrightarrow Z^{\log }$ with $f^{\log }$. If, moreover, $\gamma$ is non-nodal, then such a maximal representative $f^{\log }: Z^{\log } \hookrightarrow Y^{\log }$ arises from an isomorphism of $Z^{\log }$ onto some connected component of $Y_{\mathrm{sm}}^{\log }$.

Proof. First, let us observe that the inequality $\operatorname{dim}^{\mathrm{sm}}\left(Y^{\log }\right) \leq 1$ of Definition 3.1, (i-a), together with the restriction imposed by Definition 3.1, (i-b) [cf. also Propositions 1.4, (iv); 1.6, (i)], imply that the integers " $d$ " and " $n$ " in Proposition 1.10 satisfy the following conditions:

$$
\begin{aligned}
& \left(*_{1}\right) n \in\{1,2\} ; \\
& \left(*_{2}\right) n=2 \Rightarrow d=0 ; \\
& \left(*_{3}\right) n=1 \Rightarrow d \leq 1 .
\end{aligned}
$$

Assertion (i) thus follows from $\left(*_{1}\right)$ [cf. also Lemma 1.9]. Assertion (ii) follows from $\left(*_{1}\right),\left(*_{2}\right)$ [cf. also Lemma 1.9]. Assertion (iii) follows from $\left(*_{1}\right),\left(*_{3}\right)$, together with Definition 3.1, (i-c) [cf. also Proposition 2.2, (i)]. 
Next, we consider assertion (iv). If $y$ is a nodal point of $Y^{\log }$, then write $y^{\log }$ for the $\log$ scheme obtained by restricting the $\log$ structure of $Y^{\log }$ to the closed subscheme, equipped with the reduced induced scheme structure, of $Y$

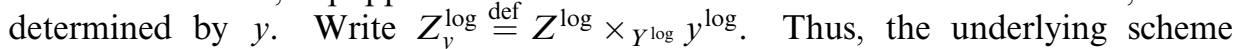
$Z_{y}$ of $Z_{y}^{\log }$ may be identified with the scheme-theoretic fiber of $Z$ over $y$. Note that if $Z_{y}=\emptyset$ for every nodal point $y$ of $Y^{\log }$, then $f^{\log }$ admits a factorization $Z^{\log } \hookrightarrow Y_{\mathrm{sm}}^{\log } \hookrightarrow Y^{\log }$ as the composite of a monomorphism $Z^{\log } \hookrightarrow Y_{\mathrm{sm}}^{\log }$ with the natural monomorphism $Y_{\mathrm{sm}}^{\log } \leadsto Y^{\log }$; moreover, since $f^{\log }$ is $S L E M$, it follows immediately that the morphism $Z^{\log } \hookrightarrow Y_{\mathrm{sm}}^{\log }$ is $S L E M$ and hence, by assertion (ii) and Proposition 2.2, (ii), an open immersion. Thus, since, by assertion (iii), $Z_{\text {red }}$ is regular and of dimension one, it follows immediately-i.e., by possibly replacing $Z^{\log }$ by the $\log$ scheme determined by a suitable dense open subscheme of $Z-$ that, to complete the proof of assertion (iv), it suffices to verify, under the additional assumption that $Z_{y}^{\log }$ is connected [hence nonempty] for some fixed nodal point $y$ of $Y^{\log }$, that $\operatorname{dim}\left(Z_{y}\right)=1$. To this end, let us first observe that the natural morphism $Z_{y}^{\log } \rightarrow y^{\log }$ is SLEM. Since $Z_{y}^{\log }$ is connected and [by assertion (iii)] of rank one, it follows from Lemma 1.5 , (v) [where we take " $S$ log", to be $y^{\log }$, that the monomorphism $Z_{y}^{\log } \rightarrow y^{\log }$ admits a factorization as a composite of monomorphisms

$$
Z_{y}^{\log } \mapsto y_{Z}^{\log } \hookrightarrow y^{\log }
$$

- where $y_{Z}^{\log }$ is, in the notation of Lemma 1.5, (v), a log scheme of rank one of the form " $S^{\log }[\xi]$ "; $y_{Z}^{\log } \hookrightarrow y^{\log }$ is the composite monomorphism of Lemma 1.5, (vi) [where we take " $S_{+}^{\log }[\xi] \mapsto S^{\log }[\xi]$ " to be the identity morphism]. Since $Z_{y}^{\log } \leadsto y^{\log }$ is $S L E M$, it follows immediately that $Z_{y}^{\log } \leadsto y_{Z}^{\log }$ is $S L E M$ and hence, by Proposition 2.2, (ii), an open immersion. Since the underlying scheme of $y_{Z}^{\log }$ is of dimension one [cf. Lemma 1.5, (iv), (v)], we thus conclude that $\operatorname{dim}\left(Z_{y}\right)=1$, as desired. This completes the proof of assertion (iv).

Next, we consider assertion (v). Write $k$ for the residue field of $Y$ at $y$,

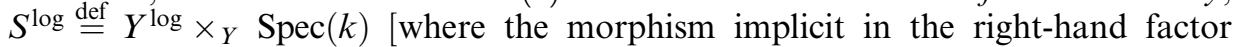
of the fiber product is the tautological morphism $\operatorname{Spec}(k) \rightarrow Y$ associated to $y$ ]. Thus, $S^{\log }$ is a $\log$ scheme of the sort that appears in Lemma 1.5 , so, in the following discussion, we shall apply the notational conventions introduced at the beginning of Lemma 1.5. Write $\mathcal{O}_{\hat{Y}}$ for the complete noetherian local ring

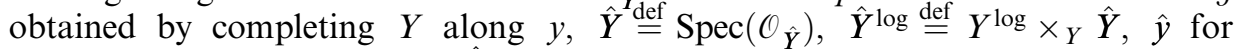
the unique closed point of $\hat{Y}$. Write $\mathcal{O}_{\hat{Y} \text { sep }}$ for the completion of the strict henselization of $\mathcal{O}_{\hat{Y}}$ determined by $k^{\text {sep }}, \hat{Y}^{\text {sep }} \stackrel{\text { def }}{=} \operatorname{Spec}\left(\mathcal{O}_{\hat{Y} \text { sep }}\right)$ [so $\hat{Y}^{\text {sep }}$ is equipped

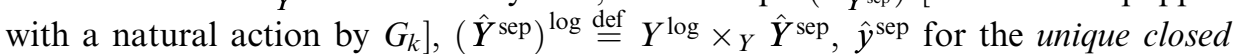
point of $\hat{Y}^{\text {sep }}$.

Next, let us fix a chart $P \rightarrow \mathcal{O}_{\hat{Y} \text { sep }}$ of $\left(\hat{Y}^{\text {sep }}\right)^{\log }$ that determines a "clean chart" in the sense of [3], Definition 1.3. This chart thus determines a natural isomorphism of the fiber at $\hat{y}^{\text {sep }}$ of the monoid $M_{\hat{Y}_{\text {sep }}}$ that defines the log structure of $\left(\hat{Y}^{\text {sep }}\right)^{\log }$ with the product $P \times \mathcal{O}_{\hat{Y} \text { sep }}^{\times}$. In particular, the natural action of $G_{k}$ on this fiber determines an action of $G_{k}$ on $P \times \mathcal{O}_{\hat{Y} \text { sep }}^{\times}$[i.e., which is compatible 
with the factor $\{0\} \times \mathcal{O}_{\hat{Y} \text { sep }}^{\times}$, but not necessarily compatible with the factor $P \times\{1\}$, of this product decomposition!], hence also on the groupification $P^{\mathrm{gp}} \times \mathcal{O}_{\hat{Y} \text { sep }}^{\times}$of $P \times \mathcal{O}_{\hat{Y} \text { sep }}^{\times}$. Note that since $Y^{\log }$ is a log-Dedekind object of $\operatorname{Sch}^{\log }\left(X^{\log }\right)$, it follows immediately from assertion (ii) that the support of the closed subscheme $\hat{Y}_{*}^{\text {sep }} \subseteq \hat{Y}^{\text {sep }}$ determined by the ideal generated by the image via the chart under consideration of $P \backslash\{0\}$ is equal to $\left\{\hat{y}^{\text {sep }}\right\}$.

Next, let

$$
Q \subseteq P^{\mathrm{gp}}
$$

be a finitely generated, saturated submonoid such that $P \subseteq Q \neq P^{\mathrm{gp}}$. Write $G_{Q} \subseteq G_{k}$ for the open subgroup of elements that preserve $Q$ [i.e., relative to the natural action of $G_{k}$ on the quotient $\left(P^{\mathrm{gp}} \times \mathcal{O}_{\hat{Y} \text { sep }}^{\times}\right) / \mathcal{O}_{\hat{Y} \text { sep }}^{\times} \stackrel{\sim}{\rightarrow} P$ gp determined, as discussed above, by the chart under consideration!]. Thus, the action of $G_{k}$ on $P^{\mathrm{gp}} \times \mathcal{O}_{\hat{Y}}^{\times}$sep determines an action of $G_{Q} \subseteq G_{k}$ on the submonoid $Q \times \mathcal{O}_{\hat{Y} \text { sep }}^{\times} \subseteq$ $P^{\mathrm{gp}} \times \mathcal{O}_{\hat{Y}^{\mathrm{sep}}}^{\times}$. Moreover, we assume further that one of the following [mutually exclusive!] conditions holds:

(v-a) $G_{Q}=G_{k}$, and, moreover, the natural inclusion $P \subseteq Q$ is a sumdominating homomorphism of $f s$ monoids [cf. the discussion entitled "Generalities on monoids" in §0].

(v-b) There exists a positive homomorphism $\xi: P \rightarrow \mathbf{N}$ which induces a surjection on groupifications $\xi^{\mathrm{gp}}: P^{\mathrm{gp}} \rightarrow \mathbf{Z}$ such that $Q$ coincides with the saturation [cf. [4], Lemma 2.5, (ii)] of the submonoid of $P^{\mathrm{gp}}$ generated by $P$ and $\operatorname{Ker}\left(\xi^{\mathrm{gp}}\right)$.

Thus, even when $G_{Q} \neq G_{k}$ [which implies that condition (v-b) holds], one verifies immediately that the natural inclusion $P \subseteq Q$ is a sum-dominating homomorphism. That is to say, the natural inclusion $P \subseteq Q$ is a sum-dominating homomorphism, no matter which of the two conditions (v-a), (v-b) one assumes.

Next, let us observe that the inclusion $P \hookrightarrow Q$ determines a $\log$ étale monomorphism

$$
Z^{\log }[Q] \stackrel{\text { def }}{=} \operatorname{Spec}\left(\mathcal{O}_{\hat{Y}^{\text {sep }}}[Q]\right)^{\log } \rightarrow Z^{\log }[P] \stackrel{\text { def }}{=} \operatorname{Spec}\left(\mathcal{O}_{\hat{Y}^{\text {sep }}}[P]\right)^{\log }
$$

[cf. the construction discussed in Proposition 1.4, (ii), as well as [1], Proposition 3.4]. Thus, one verifies immediately that the actions [determined, as discussed above, by the chart under consideration!] of $G_{k}$ on $P \times \mathcal{O}_{\hat{Y} \text { sep }}^{\times}$and of $G_{Q}$ on $Q \times \mathcal{O}_{\hat{Y} \text { sep }}^{\times}$determine, respectively, actions of $G_{k}$ on $Z^{\log }[P]$ and $G_{Q}$ on $Z^{\log }[Q]$. Moreover, the chart $P \rightarrow \mathcal{O}_{\hat{Y}^{\text {sep }}}$ under consideration determines a tautological $G_{k^{-}}$ equivariant morphism $\left(\hat{Y}^{\mathrm{sep}}\right)^{\log } \rightarrow Z^{\log }[P]$ and hence a fiber product [of fs $\log$ schemes]

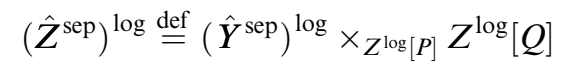

equipped with a natural action by $G_{Q}$. This natural $G_{Q}$-action in turn determines descent data for the projection morphism $\left(\hat{Z}^{\text {sep }}\right)^{\log } \rightarrow\left(\hat{Y}^{\text {sep }}\right)^{\log }$, which may 
be used to descend this projection morphism to a log étale monomorphism $\hat{Z}^{\log } \rightarrow \hat{Y}_{Q}^{\log }$, where we write $\hat{Y}_{Q} \rightarrow \hat{Y}$ for the finite étale covering corresponding to the open subgroup $G_{Q} \subseteq G_{k}, \hat{Y}_{Q}^{\log } \stackrel{\text { def }}{=} \hat{Y}^{\log } \times_{\hat{Y}} \hat{Y}_{Q}$.

Next, let us observe that since $Y^{\log }$ is a $\log$-Dedekind object of $\operatorname{Sch}^{\log }\left(X^{\log }\right)$ [or, equivalently, of $\operatorname{Sch}^{\log }\left(Y^{\log }\right)$ ], it follows immediately from assertions (ii) and (iii) that any minimal object of $\operatorname{Sch}^{\log }\left(\hat{Z}^{\log }\right)$ is of rank one. Thus, since the inclusion $P \subseteq Q$ is sum-dominating, it follows from the final portion of Lemma 1.9 that any regular function on the underlying scheme $\hat{Z}^{\text {sep }}$ of $\left(\hat{Z}^{\text {sep }}\right)^{\log }$ that arises [i.e., via the various charts implicit in the above discussion] from an element $\in P \backslash\{0\}$ necessarily vanishes at every point of $\hat{Z}^{\text {sep }}$, hence [since $\hat{Z}^{\text {sep }}$ is noetherian] is necessarily nilpotent. Since, as observed above, the support of the closed subscheme $\hat{Y}_{*}^{\text {sep }} \subseteq \hat{Y}^{\text {sep }}$ is equal to $\left\{\hat{y}^{\text {sep }}\right\}$, we thus conclude that the natural morphism $\hat{Z}^{\text {sep }} \rightarrow \hat{Y}^{\text {sep }}$ factors through a closed subscheme of $\hat{Y}^{\text {sep }}$ whose support is equal to $\left\{\hat{y}^{\text {sep }}\right\}$. This in turn implies that, if we write $\hat{Z}$ for the underlying scheme of $\hat{Z}^{\log }$, then the composite morphism $\hat{Z} \rightarrow \hat{Y}_{Q} \rightarrow \hat{Y}$ factors through a closed subscheme of $\hat{Y}$ whose support is equal to $\{\hat{y}\}$.

Next, I claim that the composite morphism

$$
\hat{Z}^{\log } \rightarrow \hat{Y}_{Q}^{\log } \rightarrow \hat{Y}^{\log }
$$

is a $\log$ étale monomorphism. Indeed, in light of what has already been verified, it suffices to prove, in the case where $G_{Q} \neq G_{k}$ [which implies that condition (v-b) holds], that this composite morphism is a monomorphism. Since the morphism $\hat{Z}^{\log } \rightarrow \hat{Y}_{Q}^{\log }$ is already known to be a monomorphism, and the morphism $\hat{Y}_{Q}^{\log } \rightarrow$ $\hat{Y}^{\log }$ is a scheme-like morphism whose underlying morphism of schemes is finite étale, one verifies immediately that to complete the proof of the claim, it suffices to verify [cf. the argument applied in the proof of Lemma 1.5, (vi); the fact that the composite morphism $\hat{Z} \rightarrow \hat{Y}_{Q} \rightarrow \hat{Y}$ factors through a closed subscheme of $\hat{Y}$ whose support is equal to $\{\hat{y}\}]$ that the base-change of the morphism $\hat{Z}^{\log } \rightarrow \hat{Y}^{\log }$ via the natural morphism $S^{\log } \rightarrow \hat{Y}^{\log }$ is a monomorphism. On the other hand, one verifies immediately that this base-changed morphism $\hat{Z}^{\log } \times{ }_{\hat{Y} \log } S^{\log } \rightarrow S^{\log }$

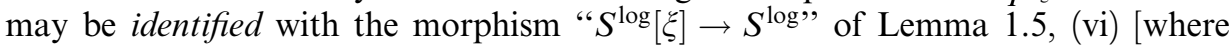
the objects " $\xi$ ", " $H$ " of Lemma 1.5, (vi), correspond, respectively, to $\xi$ and $G_{Q}$ in the present discussion; we observe that it follows immediately from condition (v-b) that " $\Xi_{+}=\Xi$ ']. Thus, the fact that this base-changed morphism $\hat{Z}^{\log } \times_{\hat{Y} \log }$ $S^{\log } \rightarrow S^{\log }$ is a monomorphism follows from Lemma 1.5 , (vi). This completes the proof of the claim.

Thus, in summary, the composite morphism $\hat{Z}^{\log } \rightarrow \hat{Y}^{\log } \rightarrow Y^{\log }$ may be regarded as a $\log$ étale monomorphism of $\operatorname{Sch}^{\log }\left(Y^{\log }\right)$, or, indeed, of $\operatorname{Sch}^{\log }\left(X^{\log }\right)$. In the following, we shall use the notation

$$
f^{\log }: Z^{\log } \rightarrow Y^{\log }
$$

to denote this composite morphism. Moreover, one computes easily that, if we write $Z$ for the underlying scheme of $Z^{\log }$, then $Z_{\text {red }} \times_{\hat{Y}_{Q}} \hat{Y}^{\text {sep }}$ may be identified with the reduced closed subscheme of $\operatorname{Spec}\left(k^{\operatorname{sep}}[Q]\right)$ determined by forming the 
zero locus of the set of functions $P \backslash\{0\} \subseteq Q$. Thus, if condition (v-a) holds, then one verifies immediately, by applying an isomorphism $Q^{\text {pf } \stackrel{\sim}{\rightarrow}} \mathbf{Q}_{\geq 0} \oplus \mathbf{Q}_{\geq 0}$ as in the discussion entitled "Rank two fs monoids" in $§ 0$ [cf. also Lemma 1.5, (iv)], that $Z_{\text {red }} \times_{\hat{Y}_{Q}} \hat{Y}^{\text {sep }}$ may be regarded as the codomain of a finite surjective morphism whose domain consists of two copies of the affine line over $k^{\text {sep }}$ glued together at a single point, hence, in particular, is connected. On the other hand, if condition (v-b) holds, then one verifies immediately that $Z_{\text {red }} \times_{\hat{Y}_{Q}} \hat{Y}^{\text {sep }}$ is a one-dimensional torus [cf. the situation discussed in Lemma 1.5, (iv)], hence, in particular, is connected.

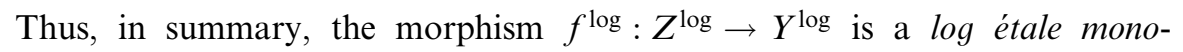
morphism with connected domain such that the resulting chain

$$
\operatorname{Chn}\left(f^{\log }\right) \subseteq \operatorname{SmCp}\left(Y^{\log }\right)
$$

is contained in $\operatorname{SmCp}\left(Y^{\log }\right)_{y}$. Now we consider the monoids constructed in Example 0.2, where we allow $n \in \mathbf{N}$ to vary. Then it follows immediately from the discussion of Example 0.2 that given any element $\gamma \in \operatorname{SmCp}\left(Y^{\log }\right)_{y}$, it holds that $\gamma \in \operatorname{Chn}\left(f^{\log }\right)$, if, in the notation of Example 0.2 , we take $Q \stackrel{\text { def }}{=}{ }^{n} P-\mathrm{a}$ submonoid which, as discussed in Example 0.2, may be constructed in such a way that condition (v-a) holds-for $n$ sufficiently large.

Finally, let $H^{\log } \rightarrow Y^{\log }$ be a morphism in $\operatorname{Sch}^{\log }\left(X^{\log }\right)$ from a minimal object $H^{\log }$ to $Y^{\log }$ that maps the unique point of the underlying scheme $H$ of $H^{\log }$ to $y$. Thus, if we regard $H$ as the spectrum of a finite subextension of $k$ in the perfection of $k^{\text {sep }}$, then the morphism $H^{\log } \rightarrow Y^{\log }$ determines, by considering the induced morphism on $\log$ structures, a positive homomorphism $\xi: P \rightarrow \mathbf{N}$ and submonoid $Q \subseteq P^{\mathrm{gp}}$ that satisfy condition (v-b). Moreover, it follows immediately from the construction of $f^{\log }$ that $Z^{\log }$ is submonic [so $f^{\log }$ may be regarded as a representative of an element of $\left.\operatorname{SmCp}\left(Y^{\log }\right)_{y}\right]$, and that the morphism $H^{\log } \rightarrow Y^{\log }$ factors through $f^{\log }$. This completes the proof of assertion (v).

Finally, we consider assertion (vi). If $\gamma$ is non-nodal, then assertion (vi) follows immediately from assertions (iii) and (iv). Thus, we may assume without loss of generality that $\gamma$ is nodal. Then assertion (vi) follows immediately by gluing, in the notation of Definition 3.1, (ii), the various $Z_{i}^{\log } \neg Y^{\log }$ that constitute an element of $\operatorname{SmCp}\left(Y^{\log }\right)$ along the open immersions $Z_{12}^{\log } \mapsto Z_{i}^{\log }$. Here, we note that it follows immediately from the fact that the $\log$ scheme $y_{Z} \log$ that appeared in the proof of assertion (iv) is noetherian that this gluing process terminates after a finite number of steps. This completes the proof of assertion (vi).

Definition 3.3. Suppose that $Y^{\log }$ is a connected, non-submonic, logDedekind object of $\operatorname{Sch}^{\log }\left(X^{\log }\right)$. Let $\gamma \in \operatorname{SmCp}\left(Y^{\log }\right)$. Write

$$
\operatorname{Mono}\left(Y^{\log }\right)
$$

for the full subcategory of $\operatorname{Sch}^{\log }\left(Y^{\log }\right)$ determined by the arrows $H^{\log } \rightarrow Y^{\log }$ of $\operatorname{Sch}^{\log }\left(X^{\log }\right)$ which are monomorphisms in $\operatorname{Sch}^{\log }\left(X^{\log }\right)$. 
(i) Let $C_{1}, C_{2} \subseteq \operatorname{SmCp}\left(Y^{\log }\right)$ be chains. Then we shall say that the pair of chains $\left\{C_{1}, C_{2}\right\}$ forms a partition at $\gamma$ if the chains $C_{1}, C_{2}$ satisfy the following conditions:

(i-a) $C_{1} \cup C_{2}=\operatorname{SmCp}\left(Y^{\log }\right), C_{1} \cap C_{2}=\{\gamma\}$;

(i-b) for $i=1,2$, the subset $C_{i} \backslash\{\gamma\} \subseteq \operatorname{SmCp}\left(Y^{\log }\right)$ is an $\mathbf{N}$-chain [hence nonempty];

(i-c) the $\mathbf{N}$-chains of (i-b) are "maximal" in the sense that every $\mathbf{N}$-chain $C \subseteq \operatorname{SmCp}\left(Y^{\log }\right)$ such that $\gamma \notin C$ is contained in $C_{i}$ for some $i \in\{1,2\}$;

(i-d) if, for $i=1,2$, we write $\Psi_{i}$ for the subfunctor of the contravariant functor determined by the terminal object [i.e., $Y^{\log }$ ] of $\operatorname{Mono}\left(Y^{\log }\right)$ that consists of objects $h^{\log }: H^{\log } \neg Y^{\log }$ of $\operatorname{Mono}\left(Y^{\log }\right)$ such that every composite morphism $H_{*}^{\log } \hookrightarrow H^{\log } \hookrightarrow Y^{\log }$, where $H_{*}^{\log } \mapsto H^{\log }$ is a minimal point of $H^{\mathrm{log}}$, factors through some representative of an element $\in C_{i}\left(\subseteq \operatorname{SmCp}\left(Y^{\log }\right)\right)$, then $\Psi_{i}$ is representable by an object $h_{i}^{\log }: Y_{i}^{\log } \mapsto Y^{\log }$ of $\operatorname{Mono}\left(Y^{\log }\right)$.

We shall say that $Y^{\log }$ is orientable if $Y^{\log }$ admits a partition at every element of $\operatorname{SmCp}\left(Y^{\log }\right)$.

(ii) Let $\left\{C_{1}, C_{2}\right\}$ be a partition at $\gamma$. Suppose that $h_{1}^{\log }, h_{2}^{\log }$ are as in (i-d). Then we shall say that the partition $\left\{C_{1}, C_{2}\right\}$ is seamless if the following condition is satisfied:

a monomorphism $h^{\log }: H^{\log } \hookrightarrow Y^{\log }$ in $\operatorname{Sch}^{\log }\left(X^{\log }\right)$ is an isomorphism if and only if, for $i=1,2$, the projection $H^{\log } \times_{Y^{\log }} Y_{i}^{\log } \rightarrow Y_{i}^{\log }$ associated to the fiber product determined by $h^{\log }$ and $h_{i}^{\log }$ is an isomorphism.

We shall say that $Y^{\log }$ is homogeneous if $Y^{\log }$ is orientable, and, moreover, no partition at an element $\in \operatorname{SmCp}\left(Y^{\log }\right)$ is seamless.

Remark 3.3.1. In the situation of Definition 3.3, (i-d), we observe that it follows immediately from Proposition 3.2, (v), (vi), that [the underlying morphism of schemes of] the morphism

$$
h_{1}^{\log } \amalg h_{2}^{\log }: Y_{1}^{\log } \amalg Y_{2}^{\log } \rightarrow Y^{\log }
$$

is surjective.

Proposition 3.4 (First properties of partitions). (i) Suppose that $Y^{\log }$ is an orientable object of $\mathrm{Sch}^{\log }\left(X^{\log }\right)$. Let $\left\{C_{1}, C_{2}\right\}$ be a partition at an element $\gamma \in \operatorname{SmCp}\left(Y^{\log }\right)$. Then, up to a possible permutation of the indices "1", " 2 ", every partition at $\gamma$ coincides with $\left\{C_{1}, C_{2}\right\}$.

(ii) Suppose that $Y^{\log }$ is an orientable object of $\operatorname{Sch}^{\log }\left(X^{\log }\right)$. Let $\left\{C_{1}, C_{2}\right\}$ be a partition at a non-nodal element $\gamma \in \operatorname{SmCp}\left(Y^{\log }\right) ; h_{1}^{\log }: Y_{1}^{\log } \mapsto Y^{\log }, h_{2}^{\log }$ : $Y_{2}^{\log } \longmapsto Y^{\log }$ monomorphisms as in Definition 3.3, (i-d). Then, for $i=1,2$, 
$h_{i}^{\log }: Y_{i}^{\log } \hookrightarrow Y^{\log }$ is an open immersion, and the fiber product $Y_{1}^{\log } \times_{Y^{\log }} Y_{2}^{\log }$ determined by $h_{1}^{\log }$ and $h_{2}^{\log }$ is a maximal representative for $\gamma$, i.e., in the sense of Proposition 3.2, (vi). In particular [cf. Remark 3.3.1], the partition $\left\{C_{1}, C_{2}\right\}$ is seamless.

(iii) Suppose that $Y^{\log }$ is a homogeneous object of $\operatorname{Sch}^{\log }\left(X^{\log }\right)$. Then $Y^{\log }$ is one-pointed, and $Y_{\mathrm{sm}}^{\log }$ is empty.

(iv) Suppose that $Y^{\log }$ is a log-nodal object of $\operatorname{Sch}^{\log }\left(X^{\log }\right)$. Then $Y^{\log }$ is homogeneous, hence, in particular, orientable. Moreover, relative to the notational conventions introduced in Definition 1.1, (i), $\operatorname{SmCp}\left(Y^{\log }\right)$ may be naturally identified with the set of positive homomorphisms $\xi: P_{Y} \rightarrow \mathbf{N}$ such that $\xi$ induces a surjection on groupifications $\xi^{\mathrm{gp}}: P_{Y}^{\mathrm{gp}} \rightarrow \mathbf{Z}$.

(v) Suppose that $Y^{\log }$ is a reduced, one-pointed, non-split object of rank two of $\operatorname{Sch}^{\log }\left(X^{\log }\right)$. Then $Y^{\log }$ is log-Dedekind, but not orientable. In particular, $Y^{\log }$ is not homogeneous. If, moreover, $Y=\operatorname{Spec}\left(k_{Y}\right)$ for some field $k_{Y}$, and $k_{Z}$

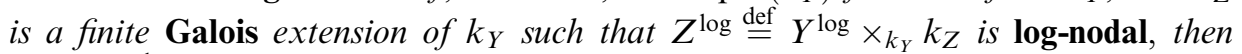
$\operatorname{SmCp}\left(Y^{\log }\right)$ may be naturally identified with the set of $\mathrm{Gal}\left(k_{Z} / k_{Y}\right)$-orbits of the set $\operatorname{SmCp}\left(Z^{\log }\right)$ [i.e., which was described explicitly in (iv)].

Proof. Assertion (i) follows, by applying entirely formal set-theoretic considerations, from Definition 3.3, (i-a), (i-b), (i-c). Next, we consider assertion (ii). If one restricts the morphisms $h_{i}^{\log }: Y_{i}^{\log } \hookrightarrow Y^{\log }$ to the open subscheme $Y_{\mathrm{sm}} \subseteq Y$ [cf. Proposition 3.2, (ii)], then one verifies immediately that the corresponding "restrictions" [in the evident sense] to $Y_{\mathrm{sm}}$ of the properties asserted in assertion (ii) follow immediately from Proposition 3.2, (vi). Next, let $y$ be a nodal point of $Y^{\log }$. Then, since $\gamma$ is non-nodal, it follows immediately from Propositions 1.6, (i); 3.2, (iv), (v); Definition 3.3, (i-a), (i-c), (i-d), that there exists a $j \in\{1,2\}$ such that, if $i=j$ (respectively, $i \neq j$ ), then $\operatorname{SmCp}\left(Y^{\log }\right)_{y} \subseteq C_{i}$ (respectively, $\left.\operatorname{SmCp}\left(Y^{\log }\right)_{y} \cap C_{i}=\emptyset\right)$, and, moreover, the restriction of $h_{i}^{\log }$ to the formal scheme obtained by completing $Y$ along $y$ is an isomorphism (respectively, has empty domain). Thus, it follows immediately [cf. Proposition 3.2, (ii)] that there exists a Zariski open neighborhood $U$ of $y$ in $Y$ such that, for $i=1,2$, the restriction $\left.h_{i}^{\log }\right|_{U}$ of $h_{i}^{\log }$ to $U$ is scheme-like, and, moreover, the underlying morphism of schemes associated to $\left.h_{i}^{\log }\right|_{U}$ is an étale monomorphism [cf. Proposition 1.4, (v)], hence, by elementary scheme theory, an open immersion, whose image contains $y$ if $i=j$. The seamlessness of the partition $\left\{C_{1}, C_{2}\right\}$ thus follows from elementary scheme theory [i.e., an easy case of "Zariski descent"]. This completes the proof of assertion (ii).

Next, we consider assertion (iii). First, let us observe that it follows formally from assertion (ii) that every submonic component of a homogeneous object of $\operatorname{Sch}^{\log }\left(X^{\log }\right)$ is necessarily nodal. It thus follows formally [cf. Proposition 3.2, (vi)] that $Y_{\mathrm{sm}}^{\mathrm{log}}$ is empty and hence, by Proposition 3.2, (ii), that $Y$ is of dimension zero. Since homogeneous objects of $\operatorname{Sch}^{\log }\left(X^{\log }\right)$ are, by definition, connected [hence nonempty], we thus conclude that $Y^{\log }$ is one-pointed. This completes the proof of assertion (iii). 
Next, we consider assertion (iv). First, let us observe that $Y^{\log }$ satisfies the hypotheses imposed on the $\log$ scheme " $S$ log" of Lemma 1.5. Thus, Lemma 1.5, (iv), (v), which we apply in the case where, in the notation of loc. cit., " $Q$ " is of

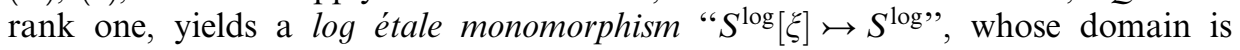
connected and submonic. In particular, it follows immediately from the existence and functorial interpretation [cf. Lemma 1.5, (iv), (v)] of such monomorphisms " $S^{\log }[\xi] \nrightarrow S^{\log "}$ that $Y^{\log }$ is $\log$-Dedekind [cf. Propositions 1.4, (vi); 2.2, (ii)]. Next, for simplicity, let us write $P \stackrel{\text { def }}{=} P_{Y}$. Then observe that, since $Y^{\log }$ is split, it follows immediately from the various definitions involved that any element $\gamma \in \operatorname{SmCp}\left(Y^{\log }\right)$ determines-i.e., by considering the morphism induced on $\log$ structures by a representative of $\gamma$ [cf. Proposition 1.4, (iii)] - a positive homomorphism $\xi_{\gamma}: P \rightarrow \mathbf{N}$ such that $\xi_{\gamma}$ induces a surjection on groupifications $\xi_{\gamma}^{\mathrm{gp}}: P^{\mathrm{gp}} \rightarrow \mathbf{Z}$. Moreover, it follows immediately from Proposition 3.2, (vi), together with the various properties of the monomorphisms " $S^{\log }[\xi] \mapsto S^{\log }$ " discussed in Lemma 1.5, (v), that the assignment

$$
\gamma \mapsto \xi_{\gamma}
$$

just discussed determines a natural bijection between $\operatorname{SmCp}\left(Y^{\log }\right)$ and the set of positive homomorphisms $\xi: P \rightarrow \mathbf{N}$ such that $\xi$ induces a surjection on groupifications $\xi^{\mathrm{gp}}: P^{\mathrm{gp}} \rightarrow \mathbf{Z}$. In the following, we shall apply this natural bijection to identify these two sets.

Next, let $\gamma \in \operatorname{SmCp}\left(Y^{\log }\right)$. Write $\phi_{0}: P \rightarrow J_{0} \stackrel{\text { def }}{=} \mathbf{N}$ for the element $\xi_{\gamma}$ discussed above. In the notation of the discussion entitled "Rank two fs monoids" in $\S 0$, for $i=1,2$, let us write $\phi_{i}: P \rightarrow J_{i}$ for the associated positive homomorphism of fs monoids [which is well-defined, up to possible permutation of the indices " 1 " and " 2 "] and $C_{i} \subseteq \operatorname{SmCp}\left(Y^{\log }\right)$ for the subset of elements $\delta \in \operatorname{SmCp}\left(Y^{\log }\right)$ such that $\xi_{\delta}: P \rightarrow \mathbf{N}$ factors through either $\phi_{0}$ or $\phi_{i}$. Then I claim that

$$
\left\{C_{1}, C_{2}\right\} \text { is a partition at } \gamma \text { which is not seamless. }
$$

Indeed, let us first observe that condition (i-a) of Definition 3.3 follows immediately from the discussion of bisecting monoids in $\$ 0$. Next, let us observe that, if we take the log scheme " $S^{\log "}$ in Lemma 1.5 to be $Y^{\log }$, then it follows, by applying Lemma 1.5, (vii), (viii), to $\phi_{0}$, that, for $i=1,2$, the $\log$ étale mono-

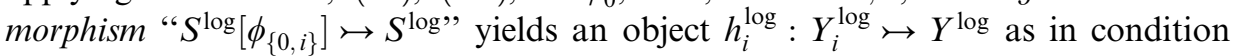
(i-d) of Definition 3.3. Next, we verify condition (i-c) of Definition 3.3. To this end, suppose that $C \subseteq \operatorname{SmCp}\left(Y^{\log }\right) \backslash\{\gamma\}$ is a chain that intersects both $C_{1} \backslash\{\gamma\}$ and $C_{2} \backslash\{\gamma\}$. Then it follows immediately from the connectedness assumption in the definition of a chain [cf. Definition 3.1, (iii)], together with Proposition 1.4, (iii); Lemma 1.9, that there exists a rank two $f_{S}$ monoid $P^{*}$ that arises as a submonoid of $P^{\mathrm{gp}}$ that contains $P$ and, moreover, for $i=1,2$, admits a homomorphism $\psi_{i}: P^{*} \rightarrow \mathbf{N}$ whose restriction to $P$ determines an element of $C_{i} \backslash\{\gamma\}$. Moreover, it follows immediately from the description given above of $\operatorname{SmCp}\left(Y^{\log }\right)$ [i.e., by considering suitable minimal points - cf. also Proposition 2.2, (ii)] that $P^{*}$ may 
be chosen so that any positive homomorphism $P^{*} \rightarrow \mathbf{N}$ that induces a surjection on groupifications determines an element of $C$. On the other hand, it follows immediately from the "continuity property" of bisecting monoids discussed in $\S 0$ that $\phi_{0}$ extends to a positive homomorphism $P^{*} \rightarrow \mathbf{N}$ and hence that $\gamma \in C$, a contradiction. This completes the verification of condition (i-c) of Definition 3.3. Next, we observe that condition (i-b) of Definition 3.3-i.e., the fact that, for $i=1,2, C_{i} \backslash\{\gamma\}$ is an $\mathbf{N}$-chain-follows immediately by considering the

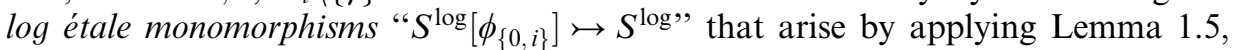
(vii), (viii) [for an appropriate choice of the indices " 1 " and " 2 "], to a sequence of bisecting monoids as in Example 0.1, where we take " $P \subseteq{ }^{\infty} P$ " to be the inclusion of monoids $P \subseteq J_{i}$ that appears in the present discussion. This completes the proof of the fact that $\left\{C_{1}, C_{2}\right\}$ is a partition at $\gamma$. The fact that this partition is not seamless follows immediately from the existence of the log étale monomorphism " $S^{\log }\left[\phi_{\{0,1,2\}}\right] \nrightarrow S^{\log "}$ that arises by applying Lemma 1.5 , (vii), (viii), to $\phi_{0}$. This completes the proof of the claim. Now it follows formally that $Y^{\log }$ is homogeneous. This completes the proof of assertion (iv).

Finally, we consider assertion (v). First, we observe that the fact that $Y^{\log }$ is log-Dedekind follows immediately from assertion (iv), via a routine étale descent argument; the description given in the statement of assertion (v) of the set $\operatorname{SmCp}\left(Y^{\log }\right)$ also follows immediately, in light of the various definitions involved, via a routine étale descent argument [cf. also Proposition 3.2, (vi)]. Now let $\delta \in \operatorname{SmCp}\left(Y^{\log }\right)$ be an element that arises from a $\operatorname{Gal}\left(k_{Z} / k_{Y}\right)$-invariant element $\gamma \in \operatorname{SmCp}\left(Z^{\log }\right)$. Here, we note that the existence of such an element of $\operatorname{SmCp}\left(Z^{\log }\right)$ follows immediately from the description of $\operatorname{SmCp}\left(Z^{\log }\right)$ given in assertion (iv), together with Lemma 1.5, (ii), which implies the existence of a suitable positive homomorphism $\xi_{\gamma}: P \stackrel{\text { def }}{=} P_{Z} \rightarrow \mathbf{N}$. Then to complete the proof that $Y^{\log }$ is not orientable, it suffices to verify that $Y^{\log }$ does not admit a partition at $\delta$. Moreover, to verify that $Y^{\log }$ does not admit a partition at $\delta$, it suffices, in light of conditions (i-b), (i-c) of Definition 3.3, to show that $\operatorname{SmCp}\left(Y^{\log }\right) \backslash\{\delta\}$ is an $\mathbf{N}$-chain.

To this end, we consider the sequence of bisecting monoids $\left\{{ }^{n} P\right\}_{n \in \mathbf{N}}$ of Example 0.1 , where we take " $P \subseteq{ }^{\infty} P$ " to be one of the two bisecting monoids of $P$ at $\xi_{\gamma}$. Thus, the homomorphism " ${ }^{\infty} \phi$ " of Example 0.1 corresponds to $\xi_{\gamma}$ in the present discussion. Now let us consider the log étale monomorphisms

$$
\text { " } S^{\log }\left[\phi_{\{0,1\}}\right] \mapsto S^{\log ,}
$$

that arise by applying Lemma 1.5, (vii), (viii), (ix), where we take the log scheme

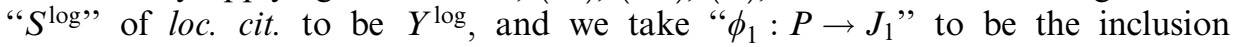
$P \subseteq{ }^{n} P$, for $n \in \mathbf{N}$. Here, we observe that if $\zeta: P \rightarrow \mathbf{N}$ and $\sigma$ are as in the condition of the display of Lemma 1.5, (ix), and $\sigma$ acts nontrivially on $P$, then it follows immediately from the $\operatorname{Gal}\left(k_{Z} / k_{Y}\right)$-invariance of $\xi_{\gamma}$ [i.e., " $\left." \phi "\right]$ that $\sigma$ acts nontrivially on $\operatorname{Ker}\left(\xi_{\gamma}^{\mathrm{gp}}\right)(\cong \mathbf{Z})$, and hence [since both $\zeta$ and $\zeta \circ \sigma$ are assumed to factor through $J_{1}$ and hence through " $\infty P$ "] that $\zeta^{\mathrm{gp}}$ vanishes on $\operatorname{Ker}\left(\xi_{\gamma}^{\mathrm{gp}}\right)$; but this implies that we may assume without loss of generality that $\zeta=\xi_{\gamma}$, which in 
turn implies [cf. Example 0.1] that $\zeta^{\mathrm{gp}}\left(J_{1}\right)=\xi_{\gamma}^{\mathrm{gp}}\left(J_{1}\right) \subseteq \mathbf{Z}$ contains both positive and negative elements, in contradiction to the assumptions imposed on $\zeta$. That is to say, the condition of the display of Lemma 1.5, (ix), is satisfied.

Thus, in summary, we obtain a collection

$$
\left\{Z_{n}^{\log } \mapsto Y^{\log }\right\}_{n \in \mathbf{N}}
$$

of log étale monomorphisms with connected domains [cf. Lemma 1.5, (vii), (viii)] such that [cf. the discussion of Example 0.1] $\delta \notin \operatorname{Chn}\left(Z_{n}^{\log } \mapsto Y^{\log }\right) \subseteq \operatorname{SmCp}\left(Y^{\log }\right)$, and, moreover, $\bigcup_{n \in \mathbf{N}} \operatorname{Chn}\left(Z_{n}^{\log } \mapsto Y^{\log }\right)=\operatorname{SmCp}\left(Y^{\log }\right) \backslash\{\delta\}$. This completes the proof of the fact that $\operatorname{SmCp}\left(Y^{\log }\right) \backslash\{\delta\}$ is an $\mathbf{N}$-chain and hence of assertion (v).

Proposition 3.5 (Characterization of log-nodal objects).

(i) Suppose that $Y^{\log }$ is nonempty object of $\operatorname{Sch}^{\log }\left(X^{\log }\right)$. Then $Y^{\log }$ is onepointed if and only if the following condition is satisfied:

For $i=1,2$, let $U_{i}^{\log }$ be a minimal object of $\operatorname{Sch}^{\log }\left(X^{\log }\right)$ and $f_{i}^{\log }$ : $U_{i}^{\log } \rightarrow Y^{\log }$ an arrow of $\operatorname{Sch}^{\log }\left(X^{\log }\right)$. Then there exist a morphism $f_{W}^{\log }: W^{\log } \rightarrow Y^{\log }$ and, for each $i=1,2$, a morphism $h_{i}^{\log }: V_{i}^{\log } \rightarrow U_{i}^{\log }$ between minimal objects of $\operatorname{Sch}^{\log }\left(X^{\log }\right)$ such that $W^{\log }$ is homogeneous, and, moreover, for each $i=1,2$, the composite morphism $f_{i}^{\log } \circ h_{i}^{\log }$ : $V_{i}^{\log } \rightarrow Y^{\log }$ admits a factorization $V_{i}^{\log } \rightarrow W^{\log } \rightarrow Y^{\log }$ through $f_{W}^{\log }$ : $W^{\log } \rightarrow Y^{\log }$.

(ii) Suppose that $Y^{\log }$ is an object of $\operatorname{Sch}^{\log }\left(X^{\log }\right)$. Then $Y^{\log }$ is log-nodal if and only if $Y^{\log }$ is homogeneous, and the identity morphism $Y^{\log } \rightarrow Y^{\log }$ is a minimal point-hull in $\mathrm{Sch}^{\log }\left(X^{\log }\right)$.

Proof. First, we consider assertion (i). Since, by Proposition 3.4, (iii), homogeneous objects are one-pointed, one verifies immediately from the sufficency portion of Proposition 2.10, (iii), that the condition under consideration implies that $\mathrm{PtCl}\left(Y^{\log }\right)$ is of cardinality one, and hence, by Proposition 2.10, (i), (iv), that $Y^{\log }$ is one-pointed, as desired. Now suppose that $Y^{\log }$ is one-pointed. Then, by Proposition 2.10, (i), (iv), it follows that $\mathrm{PtCl}\left(Y^{\log }\right)$ is of cardinality one. Since, by Proposition 3.4, (iv), log-nodal objects are homogeneous, we thus conclude from the definition of the notion of "point-equivalence" that the condition under consideration is satisfied. This completes the proof of assertion (i).

Next, we consider assertion (ii). The necessity portion of assertion (ii) follows immediately from Propositions 2.10, (v); 3.4, (iv). The sufficiency portion of assertion (ii) follows immediately, in light of the definition of the term "homogeneous", from Propositions 2.10, (v); 3.2, (i); 3.4, (v). This completes the proof of assertion (ii).

THEOREM 3.6 (Reconstruction of the scheme structure of arbitrary objects). For $i=1,2$, let $X_{i}^{\log }$ be a locally noetherian fs log scheme [cf. the discussion 
entitled "Log schemes" in §0]. For $i=1,2$, we shall write $\operatorname{Sch}^{\log }\left(X_{i}^{\log }\right)$ for the category defined at the beginning of $\$ 1$. Let

$$
\Phi: \operatorname{Sch}^{\log }\left(X_{1}^{\log }\right) \stackrel{\sim}{\rightarrow} \operatorname{Sch}^{\log }\left(X_{2}^{\log }\right)
$$

be an [arbitrary!] equivalence of categories. Then:

(i) $\Phi$ preserves the following:

(i-a) log-Dedekind objects;

(i-b) the set $\mathrm{SmCp}(-)$ associated to a log-Dedekind object;

(i-c) the subsets of the set $\mathrm{SmCp}(-)$ of $(\mathrm{i}-\mathrm{b})$ which are [N-]chains;

(i-d) partitions at elements of the set $\mathrm{SmCp}(-)$ of (i-b);

(i-e) orientable objects;

(i-f) homogeneous objects;

(i-g) one-pointed objects;

(i-h) point-hulls with one-pointed codomains;

(i-i) minimal point-hulls with one-pointed codomains;

(i-j) log-nodal objects.

(ii) For $i=1,2$, let $Y_{i}^{\log }$ be an object of $\operatorname{Sch}^{\log }\left(X_{i}^{\log }\right)$; write $Y_{i}$ for the underlying scheme of $Y_{i}^{\log }$. Suppose further that $\Phi\left(Y_{1}^{\log }\right)=Y_{2}^{\log }$. Then $\Phi$ induces an equivalence of categories

$$
\left.\left.\left(\operatorname{Sch}\left(Y_{1}\right) \stackrel{\sim}{\rightarrow}\right) \operatorname{Sch}^{\log }\left(Y_{1}^{\log }\right)\right|_{\text {sch-lk }} \stackrel{\sim}{\rightarrow} \operatorname{Sch}^{\log }\left(Y_{2}^{\log }\right)\right|_{\text {sch-lk }}\left(\stackrel{\sim}{\rightarrow} \operatorname{Sch}\left(Y_{2}\right)\right)
$$

- where the equivalences in parentheses are the natural equivalences of Definition 1.1, (iv) - that is functorial [in the evident sense!] with respect to $Y_{1}^{\log }, Y_{2}^{\log }$. Finally, the composite of the equivalences of categories in the above display induces, by applying [4], Theorem 1.7, (ii), an isomorphism of schemes

$$
Y_{1} \stackrel{\sim}{\rightarrow} Y_{2}
$$

that is functorial [in the evident sense!] with respect to $Y_{1}^{\log }, Y_{2}^{\log }$.

Proof. First, we consider assertion (i). The preservation of (i-a) follows immediately from the preservation of (i-b), (i-d), (i-g), (i-h), (i-i), (i-l) asserted in Theorem 2.6, (i), together with the isomorphisms of schemes obtained in Theorem 2.6, (ii). The preservation of (i-b) follows immediately from the preservation of (i-b), (i-c), (i-h), (i-i) asserted in Theorem 2.6, (i). The preservation of (i-c) follows immediately, in light of the preservation of (i-b), from the preservation of (i-c), (i-h) asserted in Theorem 2.6, (i). The preservation of (i-d) follows immediately, in light of the preservation of (i-a), (i-b), (i-c), from the preservation of (i-a), (i-c), (i-e), (i-i) asserted in Theorem 2.6, (i). The preservation of (i-e) follows formally from the preservation of $(i-b),(i-d)$. The preservation of (i-f) follows formally from the preservation of (i-b), (i-d), (i-e), together with the preservation of (i-a) asserted in Theorem 2.6, (i). The preservation of (i-g) follows immediately, in light of the preservation of (i-f) and the characterization given in Proposition 3.5, (i), from the preservation of (i-b), (i-d) asserted in Theorem 2.6, (i). The preservation of (i-h), (i-i) follows immediately, in light of 
the preservation of (i-g), from the preservation of (i-a), (i-d) asserted in Theorem 2.6, (i). The preservation of $(\mathrm{i}-\mathrm{j})$ follows immediately from the preservation of (i-f), (i-i), together with the characterization given in Proposition 3.5, (ii). Finally, assertion (ii) follows formally, in light of the portion of assertion (i) concerning the preservation of $(\mathrm{i}-\mathrm{j})$, from Corollary 2.12, (ii).

It remains to reconstruct, in a category-theoretic fashion, the log structures of the various log schemes under consideration. The approach taken in the present paper is essentially similar to the approach taken in [4], but is formulated in a slightly different way. We begin by introducing notation as in the discussion preceding [4], Lemma 2.16: Write $\mathbf{A}_{\mathbf{Z}}^{1}=\operatorname{Spec}(\mathbf{Z}[t])$ [where $t$ is an indeterminate] for the affine line over $\mathbf{Z} ; \mathbf{A}_{\mathbf{Z}}^{\log }$ for the affine line $\mathbf{A}_{\mathbf{Z}}^{1}$ over $\mathbf{Z}$ equipped with the $\log$ structure determined by the divisor $V(t)$ [i.e., "the origin"]; $\exp _{\mathbf{A}}: \mathbf{A}_{\mathbf{Z}}^{\log } \rightarrow \mathbf{A}_{\mathbf{Z}}$ for the natural morphism determined by "forgetting the log structure";

$$
\exp _{Y^{\log }}: \mathbf{A}_{Y^{\log }}^{\log } \rightarrow \mathbf{A}_{Y^{\log }}
$$

for the "exponentiation morphism" obtained by base-changing $\exp _{\mathbf{A}}$ via the natural morphism $Y^{\log } \rightarrow \operatorname{Spec}(\mathbf{Z})$;

$$
\mathbf{A}_{Y \log }^{\times} \hookrightarrow \mathbf{A}_{Y^{\log }}
$$

for the open immersion determined by the complement of the origin of $\mathbf{A}_{Y \log } ; \mathbf{A}_{Y}^{\times}$, $\mathbf{A}_{Y}$ for the underlying schemes of $\mathbf{A}_{Y^{\log }}^{\times}, \mathbf{A}_{Y^{\log }}$;

$$
0_{Y}: Y \rightarrow \mathbf{A}_{Y}, \quad 1_{Y}: Y \rightarrow \mathbf{A}_{Y}
$$

for the sections determined by the assignments $t \mapsto 0, t \mapsto 1$. Thus, the map induced by $\exp _{Y^{\log }}$ on $Y^{\log }$-valued points may be naturally identified with $\exp _{Y}$ : $M_{Y} \rightarrow \mathcal{O}_{Y}$. Moreover, one verifies easily that the morphism $\mathbf{A}_{\mathbf{Z}} \times_{\mathbf{Z}} \mathbf{A}_{\mathbf{Z}} \rightarrow \mathbf{A}_{\mathbf{Z}}$ that defines the multiplication operation on the ring scheme $\mathbf{A}_{\mathbf{Z}} \rightarrow \operatorname{Spec}(\mathbf{Z})$ determines a morphism of log schemes over $Y^{\log }$

$$
\mathbf{A}_{Y^{\log }}^{\log } \times_{Y^{\log }} \mathbf{A}_{Y^{\log }}^{\log } \rightarrow \mathbf{A}_{Y^{\log }}^{\log }
$$

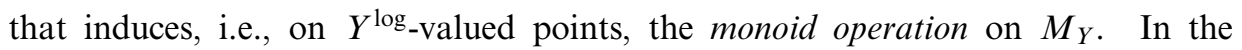
following,

we shall always regard $\mathbf{A}_{Y \log }$ as being equipped with the "ring $\log$ scheme" structure-i.e., the ring object structure in the category of log schemes-determined by the ring scheme structure of $\mathbf{A}_{\mathbf{Z}} \rightarrow \operatorname{Spec}(\mathbf{Z})$.

One verifies immediately that any automorphism of the log scheme $\mathbf{A}_{Y^{\log }}$ that lies over the identity automorphism of $Y^{\log }$ and is compatible with the ring $\log$ scheme structure of $\mathbf{A}_{Y^{\log }}$ is necessarily equal to the identity automorphism. Finally, if $Y^{\log }$ is an object of $\operatorname{Sch}^{\log }\left(X^{\log }\right)$, then we observe that $\exp _{Y^{\log }}$ : $\mathbf{A}_{Y \log }^{\log } \rightarrow \mathbf{A}_{Y \log }$ may be regarded, in a natural way, as an arrow between objects of $\operatorname{Sch}^{\log }\left(X^{\log }\right)$. 
Proposition 3.7 (Categories of quasi-exponentiation morphisms). We maintain the notation of the above discussion. Suppose that $Y^{\log }$ is an object of

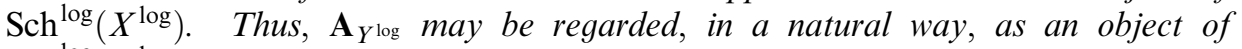
$\operatorname{Sch}^{\log }\left(X^{\log }\right)$. Write

$$
\mathrm{QExp}\left(Y^{\log }\right) \subseteq \operatorname{Sch}^{\log }\left(\mathbf{A}_{Y^{\log }}\right)
$$

for the full subcategory of $\operatorname{Sch}^{\log }\left(\mathbf{A}_{Y^{\log }}\right)$ consisting of objects $f^{\log }: Z^{\log } \rightarrow \mathbf{A}_{Y^{\log }}$ [i.e., "quasi-exponentiation morphisms"] that satisfy the following conditions:

(a) the morphism $Z^{\log } \rightarrow Y^{\log }$ determined by $f^{\log }$ is $\log$ smooth;

(b) $f^{\log }$ is log-like, i.e., induces an isomorphism $f: Z \stackrel{\sim}{\rightarrow} \mathbf{A}_{Y}$ between the underlying schemes of $Z^{\log }, \mathbf{A}_{Y^{\log }}$;

(c) the base-change of $f^{\log }$ via the open immersion $\mathbf{A}_{Y^{\log }}^{\times} \hookrightarrow \mathbf{A}_{Y^{\log }}$ is an isomorphism;

(d) if

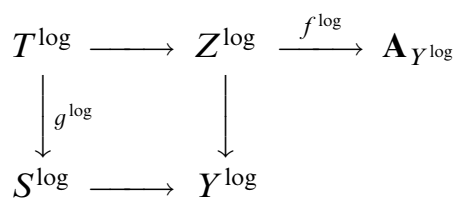

is a commutative diagram of morphisms of $\operatorname{Sch}^{\log }\left(X^{\log }\right)$ in which the horizontal arrows of the square are minimal point-hulls, and the resulting fiber product $T^{\log } \times_{\mathbf{A}_{Y^{\log }}} \mathbf{A}_{Y^{\log }}^{\times}$is the empty object of $\mathrm{Sch}^{\log }\left(X^{\log }\right)$, then $g^{\log }$ is not an isomorphism, and, moreover, if $S^{\log }$ is not a minimal object of rank zero, then, for some reduced, onepointed object $W^{\log }$ of $\operatorname{Sch}^{\log }\left(X^{\log }\right)$, there exist two distinct morphisms $h_{1}^{\log }, h_{2}^{\log }: W^{\log } \rightarrow T^{\log }$ such that the two resulting composite morphisms $g^{\log } \circ h_{1}^{\log }, g^{\log } \circ h_{2}^{\log }: W^{\log } \rightarrow T^{\log } \rightarrow S^{\log }$ coincide and are scheme-like;

(e) there exists a $Y^{\log }$-morphism $Z^{\log } \times_{Y^{\log }} Z^{\log } \rightarrow Z^{\log }$ in $\operatorname{Sch}^{\log }\left(X^{\log }\right)$ for which the induced morphism on underlying schemes coincides, relative to the isomorphism $f: Z \stackrel{\sim}{\rightarrow} \mathbf{A}_{Y}$ of condition (b), with the morphism $\mathbf{A}_{Y} \times_{Y} \mathbf{A}_{Y} \rightarrow \mathbf{A}_{Y}$ determined by the multiplication operation arising from the ring log scheme structure of $\mathbf{A}_{Y \log }$.

[Thus, $\exp _{Y^{\log }}: \mathbf{A}_{Y^{\log }}^{\log } \rightarrow \mathbf{A}_{Y^{\log }}$ may be regarded as an object of $\operatorname{QExp}\left(Y^{\log }\right)$.] Then every object $f^{\log }: Z^{\log } \rightarrow \mathbf{A}_{Y^{\log }}$ of $\mathrm{QExp}\left(Y^{\log }\right)$ is isomorphic to the object

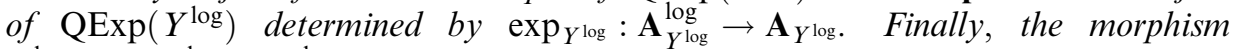
$Z^{\log } \times_{Y^{\log }} Z^{\log } \rightarrow Z^{\log }$ of condition (e) is, in fact, uniquely determined by the hypotheses imposed in condition (e).

Proof. Proposition 3.7 follows formally from [4], Lemma 2.16. Indeed, one verifies immediately that property (i) (respectively, (ii); (iii) [cf. Remark 3.7.1 below]; (iv)) of [4], Lemma 2.16, follows, in light of condition (b) in the statement 
of Proposition 3.7, from condition (c) (respectively, (d); (a); (e)) in the statement of Proposition 3.7. Here, we note in passing that the argument applied in the final paragraph of the proof of [4], Lemma 2.16, may be simplified considerably: that is to say, in the notation of loc. cit., the fact that "the morphism of monoids $Q \rightarrow P$ may be identified with the natural inclusion $Q \hookrightarrow Q \times \mathbf{N}$ " may be concluded directly from the isomorphism of rings " $k[[Q]][[T]] \stackrel{\underset{\sim}{\rightarrow}}{\rightarrow}[[P]]$ " obtained in the second to last paragraph of the proof of [4], Lemma 2.16, by considering an element $\xi \in P$ such that, if we apply this isomorphism to identify the rings $k[[Q]][[T]]$ and $k[[P]]$, then the set $Q \cup\{\xi\}$ generates the maximal ideal of the local ring $k[[P]]$.

Remark 3.7.1. In the context of Proposition 3.7, we take the opportunity to correct a misprint in the statement of [4], Lemma 2.16: In [4], Lemma 2.16, (iii), the phrase "a monomorphism" should read "a scheme-like monomorphism".

The following result may be regarded as the culmination of the theory developed in the present paper and corresponds to Theorem B [or, more precisely, Theorem 2.19, (ii)] of [4], the proof of which [i.e., as given in [4]] is, unfortunately, incomplete.

THEOREM 3.8 (Reconstruction of the log scheme structure of arbitrary objects). For $i=1,2$, let $X_{i}^{\log }$ be a locally noetherian fs log scheme [cf. the discussion entitled "Log schemes" in §0]. For $i=1,2$, we shall write $\operatorname{Sch}^{\log }\left(X_{i}^{\log }\right)$ for the category defined at the beginning of $\$ 1$. Let

$$
\Phi: \operatorname{Sch}^{\log }\left(X_{1}^{\log }\right) \stackrel{\sim}{\rightarrow} \operatorname{Sch}^{\log }\left(X_{2}^{\log }\right)
$$

be an [arbitrary!] equivalence of categories. Then:

(i) $\Phi$ preserves the following constructions [i.e., up to, in the case of (i-a), (i-c), a unique isomorphism] associated to an object "(-)":

(i-a) the ring object $\mathbf{A}_{(-)}$;

(i-b) the full subcategory $\mathrm{QExp}((-)) \subseteq \operatorname{Sch}^{\log }\left(\mathbf{A}_{(-)}\right)$;

(i-c) the exponentiation morphism $\exp _{(-)}: \mathbf{A}_{(-)}^{\log } \rightarrow \mathbf{A}_{(-)}$;

(i-d) the monoid object structure on the object $\mathbf{A}_{(-)}^{\log }$ of (i-c).

(ii) For $i=1,2$, let $Y_{i}^{\log }$ be an object of $\operatorname{Sch}^{\log }\left(X_{i}^{\log }\right)$; write $Y_{i}$ for the underlying scheme of $Y_{i}^{\log }$. Suppose further that $\Phi\left(Y_{1}^{\log }\right)=Y_{2}^{\log }$. Then $\Phi$ induces an isomorphism of log schemes

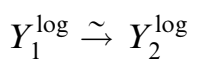

that is functorial [in the evident sense!] with respect to $Y_{1}^{\log }, Y_{2}^{\log }$ and compatible with the isomorphism of schemes of Theorem 3.6, (ii).

(iii) There exists a unique isomorphism of log schemes

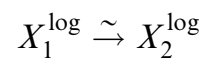

such that $\Phi$ is isomorphic to the equivalence of categories induced by this isomorphism of $\log$ schemes $X_{1}^{\log } \stackrel{\sim}{\rightarrow} X_{2}^{\log }$. 
Proof. First, we consider assertion (i). The preservation of (i-a) follows immediately from Theorem 3.6, (ii); [4], Proposition 1.6, (iii). To verify the preservation of (i-b), it suffices to verify the preservation of the conditions (a), (b), (c), (d), (e) in the statement of Proposition 3.7. The preservation of condition (a) follows immediately, in light of the functorial definition of log smoothness [i.e., in terms of scheme-like closed immersions, as in [2], \$8.1, (i)], from Theorem 3.6, (ii). The preservation of condition (b) follows formally from Theorem 3.6, (ii). The preservation of conditions (c) and (e) follows immediately from the preservation of (i-a) [i.e., which has already been verified], together with Theorem 3.6, (ii). The preservation of condition (d) follows immediately from the preservation of (i-c) [cf. also Proposition 2.10, (v)], (i-d) asserted in Corollary 2.12, (i) [which is applicable in light of the preservation of (i-j) asserted in Theorem 3.6, (i)], together with the preservation of (i-b), (i-d), (i-g) asserted in Theorem 2.6, (i). This completes the proof of the preservation of (i-b). The preservation of (i-c) and (i-d) follows formally from Proposition 3.7, together with the preservation of (i-b). This completes the proof of assertion (i).

Since the map induced by the exponentiation morphism $\exp _{(-)}$on $(-)$-valued points may be naturally identified with the morphism between sheaves of monoids that defines the log structure of " $(-)$ " [cf. the discussion preceding Proposition 3.7], assertion (ii) follows immediately from assertion (i); Theorem 3.6, (ii). Finally, assertion (iii) follows immediately from the existence of the functorial isomorphisms of log schemes discussed in assertion (ii), by considering, for $i=1,2$, a suitable ind-object of $\operatorname{Sch}^{\log }\left(X_{i}^{\log }\right)$

$$
\left\{{ }^{\alpha_{i}} Y_{i}^{\log }\right\}_{\alpha_{i} \in A_{i}}
$$

- where the transition morphisms [notation for which was omitted for the sake

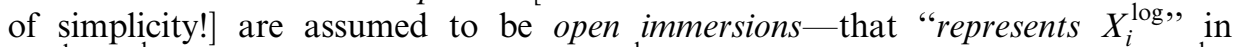
$\operatorname{Sch}^{\log }\left(X_{i}^{\log }\right)$. [Here, we recall that if $X_{i}^{\log }$ fails to be quasi-compact, then $X_{i}^{\log }$ does not determine an object of $\operatorname{Sch}^{\log }\left(X_{i}^{\log }\right)$ in the usual sense.]

\section{Section 4: Category-theoretic representation of archimedean structures}

In the present $\S 4$, we explain the relatively minor modifications to the theory developed in the present paper for log schemes that are necessary in order to accommodate categories of log schemes equipped with archimedean structures as discussed in [5]. At a more concrete level, we observe that

- Theorem 3.1;

- Proposition 4.3;

- Proposition 4.4

of [5] depend on the portions of the theory of [4] that [cf. Example 0.3; Remark 1.4.1] are in error. Thus, in the present $\$ 4$, we explain how these results, as well as the main theorem of [5] [i.e., [5], Theorem 5.1], may be repaired by applying the theory developed thus far in the present paper. 
We begin by reviewing [and slightly modifying] the notation introduced at the beginning of $[5], \S 4$. Write

\section{$\overline{\mathrm{SCH}}$}

for the category of arithmetic schemes,

\section{$\overline{\mathrm{SCH}}^{\log }$}

for the category of arithmetic log schemes [cf. [5], Definition 4.2, and the following discussion], and

$$
\mathrm{SCH} \subseteq \overline{\mathrm{SCH}} ; \quad \mathrm{SCH}^{\log } \subseteq \overline{\mathrm{SCH}}^{\log }
$$

for the full subcategories determined by the purely nonarchimedean objects [cf. [5], Definition 4.3, (i)]. Let $\bar{X}^{\log }$ be an object of $\overline{\mathrm{SCH}}^{\log }$. Thus, $\bar{X}^{\log }$ determines underlying objects $X^{\log }, \bar{X}$, and $X$ of the categories $\mathrm{SCH}^{\log }, \overline{\mathrm{SCH}}$, and $\mathrm{SCH}$, respectively. Write

$$
\begin{gathered}
\overline{\operatorname{SCH}}^{\log }\left(\bar{X}^{\log }\right) \stackrel{\text { def }}{=}\left(\overline{\mathrm{SCH}}^{\log }\right)_{\bar{X}} \log ; \quad \mathrm{SCH}^{\log }\left(X^{\log }\right) \stackrel{\text { def }}{=}\left(\mathrm{SCH}^{\log }\right)_{X} \log ; \\
\overline{\mathrm{SCH}}(\bar{X}) \stackrel{\text { def }}{=} \overline{\mathrm{SCH}}_{\bar{X}} ; \quad \mathrm{SCH}(X) \stackrel{\text { def }}{=} \mathrm{SCH}_{X}
\end{gathered}
$$

for the respective categories of "objects over the subscripted objects" [cf. the notational conventions introduced in the discussion entitled "Categories" in [5], $\S 2]$ and

$$
\begin{gathered}
\overline{\operatorname{Sch}}^{\log }\left(\bar{X}^{\log }\right) \subseteq \overline{\mathrm{SCH}}^{\log }\left(\bar{X}^{\log }\right) ; \quad \operatorname{Sch}^{\log }\left(X^{\log }\right) \subseteq \mathrm{SCH}^{\log }\left(X^{\log }\right) ; \\
\overline{\operatorname{Sch}}(\bar{X}) \subseteq \overline{\operatorname{SCH}}(\bar{X}) ; \quad \operatorname{Sch}(X) \subseteq \operatorname{SCH}(X)
\end{gathered}
$$

for the full subcategories determined by the noetherian objects. To simplify the exposition, we shall often refer to the domain of an arrow which is an object of any of the categories of the preceding display as an "object" of the category.

Note that the notation just introduced is consistent with the notational conventions introduced at the beginning of $\S 1$ of the present paper for " $\operatorname{Sch}^{\log }\left(X^{\log }\right)$ " and " $\operatorname{Sch}(X)$ ". Indeed, if $X^{\log }$ is any locally noetherian $f_{S} \log$ scheme, then one may define [in a fashion consistent with the notation introduced above!]

$$
\mathrm{SCH}^{\log }\left(X^{\log }\right)
$$

to be the category whose objects are morphisms of log schemes of locally finite type $Y^{\log } \rightarrow X^{\log }$, where $Y^{\log }$ is a locally noetherian $f_{s} \log$ scheme, and whose morphisms [from an object $Y_{1}^{\log } \rightarrow X^{\log }$ to an object $Y_{2}^{\log } \rightarrow X^{\log }$ ] are morphisms of locally finite type $Y_{1}^{\log } \rightarrow Y_{2}^{\log }$ lying over $X^{\log }$. In a similar vein, if $X$ is any locally noetherian scheme, then one may define [in a fashion consistent with the notation introduced above!]

$$
\operatorname{SCH}(X)
$$

to be the category whose objects are morphisms of schemes of locally finite type $Y \rightarrow X$, where $Y$ is a locally noetherian scheme, and whose morphisms [from an 
object $Y_{1} \rightarrow X$ to an object $\left.Y_{2} \rightarrow X\right]$ are morphisms of locally finite type $Y_{1} \rightarrow Y_{2}$ lying over $X$.

Definition 4.1. (i) We shall apply similar terminology to data [i.e., such as collections of objects and collections of morphisms] associated to any of the categories

$$
\begin{gathered}
\overline{\operatorname{Sch}}^{\log }\left(\bar{X}^{\log }\right), \quad \overline{\operatorname{SCH}}^{\log }\left(\bar{X}^{\log }\right), \quad \operatorname{Sch}^{\log }\left(X^{\log }\right), \quad \operatorname{SCH}^{\log }\left(X^{\log }\right), \\
\overline{\operatorname{Sch}}(\bar{X}), \quad \overline{\operatorname{SCH}}(\bar{X}), \quad \operatorname{Sch}(X), \quad \operatorname{SCH}(X)
\end{gathered}
$$

to the terminology that has already been established earlier in the present paper for " $\operatorname{Sch}^{\log }\left(X^{\log }\right)$ " or in [4], §1, for " $\operatorname{Sch}(X)$ " whenever this terminology may be defined in an evidently analogous fashion for the category of the above display under consideration. When it is necessary, in order to avoid confusion, to specify the category of the above display with respect to which the terminology is to be understood, we shall append an appropriate prefix such as

$$
\overline{\mathrm{Sch}}^{\log }-, \quad \overline{\mathrm{SCH}}^{\log _{-}}, \quad \mathrm{Sch}^{\log _{-}}, \quad \mathrm{SCH}^{\log _{-}}, \quad \overline{\mathrm{Sch}}-, \quad \overline{\mathrm{SCH}}-, \quad \mathrm{Sch}-, \quad \mathrm{SCH}-
$$

to the terminology in question. This convention concerning prefixes will be applied, in particular, when the terminology is to be understood as being applied to the underlying object in one of the categories of the first display that is determined by another of the categories of the first display.

(ii) Let $\overline{\mathscr{C}}^{\log } \in\left\{\overline{\mathrm{Sch}}^{\log }, \overline{\mathrm{SCH}}^{\mathrm{log}}\right\}, \bar{X}^{\log }$ an arithmetic log scheme, $\bar{Y}{ }^{\log }$ an object of $\overline{\mathscr{C}}^{\log }\left(\bar{X}^{\log }\right)$. Then we shall say that $\bar{Y}^{\log }$ is submonically nonarchimedean if it holds that every submonic one-pointed object $\bar{Z}^{\log }$ of $\overline{\mathscr{C}}^{\log }\left(\bar{X}^{\log }\right)$ that admits a morphism to $\bar{Y}^{\log }$ is purely nonarchimedean.

THEOREM 4.2 (Equivalences of categories of schemes). Let $\mathscr{C} \in\{\mathrm{Sch}, \mathrm{SCH}\}$. For $i=1,2$, let $X_{i}$ be a locally noetherian scheme. Then, relative to the notation introduced at the beginning of the present $\S 4$, any equivalence of categories

$$
\Phi: \mathscr{C}\left(X_{1}\right) \stackrel{\sim}{\rightarrow} \mathscr{C}\left(X_{2}\right)
$$

arises from $a$ unique isomorphism of schemes $X_{1} \stackrel{\sim}{\rightarrow} X_{2}$.

Proof. When $\mathscr{C}=\mathrm{Sch}$, Theorem 4.2 is precisely the content of [4], Theorem 1.7, (ii). When $\mathscr{C}=\mathrm{SCH}$, Theorem 4.2 follows from an entirely similar argument.

THEOREM 4.3 (Equivalences of categories of arithmetic schemes). Let $\overline{\mathscr{C}} \in$ $\{\overline{\mathrm{Sch}}, \overline{\mathrm{SCH}}\}$. For $i=1,2$, let $\bar{X}_{i}$ be an arithmetic scheme [cf. [5], Definition 4.2, (i)]. Then, relative to the notation introduced at the beginning of the present $\S 4$, any equivalence of categories

$$
\Phi: \overline{\mathscr{C}}\left(\bar{X}_{1}\right) \stackrel{\sim}{\rightarrow} \overline{\mathscr{C}}\left(\bar{X}_{2}\right)
$$

arises from a unique isomorphism of arithmetic schemes $\bar{X}_{1} \stackrel{\sim}{\rightarrow} \bar{X}_{2}$. 
Proof. If $\overline{\mathscr{C}}=\overline{\mathrm{Sch}}$, then set $\mathscr{C} \stackrel{\text { def }}{=} \mathrm{Sch}$; if $\overline{\mathscr{C}}=\overline{\mathrm{SCH}}$, then set $\mathscr{C} \stackrel{\text { def }}{=} \mathrm{SCH}$. Then Theorem 4.3 follows, in effect, by combining the theory of [4], §1, with the non-logarithmic portion of the theory developed in [5], $44, \S 5$. [That is to say, the errors in [5] discussed at the beginning of the present $\$ 4$ concern subtleties that arise from the $\log$ structures of the log schemes involved and hence have no effect on the non-logarithmic portion of the theory.] Indeed, let $i \in\{1,2\}$; write $X_{i}$ for the underlying scheme of $\bar{X}_{i}$. Then one verifies immediately that the $\overline{\mathscr{C}}$-minimal objects of $\overline{\mathscr{C}}\left(\bar{X}_{i}\right)$ are the purely nonarchimedean objects that arise from the $\mathscr{C}$-minimal objects of $\mathscr{C}\left(X_{i}\right)$. Thus, the one-pointed objects of $\overline{\mathscr{C}}\left(\bar{X}_{i}\right)$ are precisely the objects $\bar{Y}$ such that $\operatorname{MinPt}(\bar{Y})=\operatorname{MinPt}(Y)$ [where we write $Y$ for the object of $\mathscr{C}\left(X_{i}\right)$ determined by the underlying scheme of $\left.\bar{Y}\right]$ is of cardinality one. This characterization of one-pointed objects of $\overline{\mathscr{C}}\left(\bar{X}_{i}\right)$ allows one to circumvent the application of [5], Proposition 4.3, in the theory of [5], §4. In particular, we obtain a category-theoretic characterization of $\overline{\mathscr{C}}$-minimal point-hulls as in [5], Proposition 4.4, (iii). One thus obtains-i.e., by considering epimorphisms as in [5], Proposition 4.5-a category-theoretic characterization of the purely nonarchimedean one-pointed objects of $\overline{\mathscr{C}}\left(\bar{X}_{i}\right)$ as in [5], Corollary 4.1, (i), and of the purely archimedean morphisms [cf. [5], Definition 4.3, (ii)] of $\overline{\mathscr{C}}\left(\bar{X}_{i}\right)$ as in [5], Corollary 4.1, (ii). In particular, we obtain a category-theoretic characterization, as in [5], Corollary 4.2, of the purely nonarchimedean objects of $\overline{\mathscr{C}}\left(\bar{X}_{i}\right)$ and hence, by applying Theorem 4.2 , a category-theoretic reconstruction of the underlying scheme of an object of $\overline{\mathscr{C}}\left(\bar{X}_{i}\right)$, as in [5], Corollary 4.3. Now, to complete the proof of Theorem 4.3 [cf. the proof of [5], Theorem 5.1], it suffices to apply the "non-logarithmic global compatibility" established in [5], Lemma 5.1.

Next, we consider analogues of Theorem 2.6 for $\mathrm{SCH}^{\log }, \overline{\mathrm{Sch}}^{\log }$, and $\overline{\mathrm{SCH}}^{\log }$.

THEOREM 4.4 (Reconstruction of the scheme structure of submonic objects for $\left.\mathrm{SCH}^{\log }\right)$. For $i=1,2$, let $X_{i}^{\log }$ be a locally noetherian fs log scheme [cf. the discussion entitled "Log schemes" in §0]. We shall apply the notation introduced at the beginning of the present $\S 4$. Let

$$
\Phi: \mathrm{SCH}^{\log }\left(X_{1}^{\log }\right) \stackrel{\sim}{\rightarrow} \mathrm{SCH}^{\log }\left(X_{2}^{\log }\right)
$$

be an [arbitrary!] equivalence of categories. Then:

(i) $\Phi$ preserves the following:

(i-a) monomorphisms;

(i-b) empty objects;

(i-c) connected objects;

(i-d) minimal objects;

(i-e) minimal points;

(i-f) submonic one-pointed objects;

(i-g) ranks of minimal objects;

(i-h) SLEM morphisms;

(i-i) submonic objects; 
(i-j) scheme-like morphisms between minimal objects;

(i-k) scheme-like morphisms between submonic objects;

(i-l) the submonic dimension of objects.

(ii) For $i=1,2$, let $Y_{i}^{\log }$ be an object of $\mathrm{SCH}^{\log }\left(X_{i}^{\log }\right)$; write $Y_{i}$ for the underlying scheme of $Y_{i}^{\log }$. Suppose further that $\Phi\left(Y_{1}^{\log }\right)=Y_{2}^{\log }$. Thus, $[$ cf. the portion of (i) concerning (i-i)] $Y_{1}^{\log }$ is submonic if and only if $Y_{2}^{\log }$ is. Suppose that $Y_{i}^{\log }$ is submonic for $i=1,2$. Then $\Phi$ induces an equivalence of categories

$$
\left.\left.\left(\operatorname{SCH}\left(Y_{1}\right) \stackrel{\sim}{\rightarrow}\right) \mathrm{SCH}^{\log }\left(Y_{1}^{\log }\right)\right|_{\text {sch-lk }} \stackrel{\sim}{\rightarrow} \mathrm{SCH}^{\log }\left(Y_{2}^{\log }\right)\right|_{\text {sch-lk }}\left(\stackrel{\sim}{\rightarrow} \operatorname{SCH}\left(Y_{2}\right)\right)
$$

- where the equivalences in parentheses are the evident analogues for $\mathrm{SCH}, \mathrm{SCH}^{\log }$ of the natural equivalences of Definition 1.1, (iv) - that is functorial [in the evident sense!] with respect to $Y_{1}^{\log }, Y_{2}^{\log }$. Finally, the composite of the equivalences of categories in the above display induces, by applying Theorem 4.2, an isomorphism of schemes

$$
Y_{1} \stackrel{\sim}{\rightarrow} Y_{2}
$$

that is functorial [in the evident sense!] with respect to $Y_{1}^{\log }, Y_{2}^{\log }$.

Proof. The proof is entirely similar to the proof of Theorem 2.6.

THEOREM 4.5 (Reconstruction of the scheme structure of submonic objects for $\left.\overline{\mathrm{Sch}}^{\log }, \overline{\mathrm{SCH}}^{\mathrm{log}}\right)$. Let $\overline{\mathscr{C}}^{\log } \in\left\{\overline{\mathrm{Sch}}^{\log }, \overline{\mathrm{SCH}}^{\log }\right\} . \quad$ If $\overline{\mathscr{C}}^{\log }=\overline{\mathrm{Sch}}^{\log }$, then set $\mathscr{C}{ }^{\log } \stackrel{\text { def }}{=}$

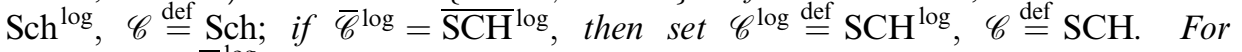
$i=1,2$, let $\bar{X}_{i}^{\log }$ be an arithmetic log scheme [cf. [5], Definition 4.2, (ii)]. We shall apply the notation introduced at the beginning of the present $\$$. Let

$$
\Phi: \overline{\mathscr{C}}^{\log }\left(\bar{X}_{1}^{\log }\right) \stackrel{\sim}{\rightarrow} \overline{\mathscr{C}}^{\log }\left(\bar{X}_{2}^{\log }\right)
$$

be an [arbitrary!] equivalence of categories. Then:

(i) $\Phi$ preserves the following:

(i-a) monomorphisms;

(i-b) empty objects;

(i-c) connected objects;

(i-d) minimal objects;

(i-e) minimal points;

(i-f) submonic one-pointed objects;

$\left(\mathrm{i}-\mathrm{f}^{\text {non }}\right)$ purely nonarchimedean submonic one-pointed objects;

(i-g) ranks of minimal objects;

(i-h) $\mathscr{C}^{\log }$-SLEM morphisms;

(i-i) submonic objects;

$\left(\mathrm{i}-\mathrm{i}^{\text {non }}\right)$ purely nonarchimedean submonic objects;

(i-j) $\mathscr{C}^{\log }$-scheme-like morphisms between minimal objects;

(i-k) $\mathscr{C}^{\log }$-scheme-like morphisms between submonic objects;

(i-1) the submonic dimension of objects. 
(ii) For $i=1,2$, let $\bar{Y}_{i}^{\log }$ be an object of $\overline{\mathscr{C}}^{\log }\left(\bar{X}_{i}^{\log }\right)$; write $Y_{i}^{\log }$ for the underlying log scheme of $\bar{Y}_{i}^{\log }, Y_{i}$ for the underlying scheme of $\bar{Y}_{i}^{\log }$. Suppose further that $\Phi\left(\bar{Y}_{1}^{\log }\right)=\bar{Y}_{2}^{\log }$. Thus, [cf. the portion of (i) concerning (i-i), (i-i $\left.\left.{ }^{\text {non }}\right)\right]$ $\bar{Y}_{1}^{\log }$ is submonic if and only if $\bar{Y}_{2}^{\log }$ is; $\bar{Y}_{1}^{\log }$ is purely nonarchimedean submonic if and only if $\bar{Y}_{2}^{\log }$ is. Suppose that $\bar{Y}_{i}^{\log }$ is submonic for $i=1,2$. Then $\Phi$ induces an equivalence of categories

$$
\left.\left.\left(\mathscr{C}\left(Y_{1}\right) \stackrel{\sim}{\rightarrow}\right) \mathscr{C}^{\log }\left(Y_{1}^{\log }\right)\right|_{\text {sch-lk }} \stackrel{\sim}{\rightarrow} \mathscr{C}^{\log }\left(Y_{2}^{\log }\right)\right|_{\text {sch-lk }}\left(\stackrel{\sim}{\rightarrow} \mathscr{C}\left(Y_{2}\right)\right)
$$

- where the equivalences in parentheses are the evident analogues for $\mathscr{C}, \mathscr{C}^{\log }$ of the natural equivalences of Definition 1.1, (iv) — that is functorial [in the evident sense!] with respect to $\bar{Y}_{1}^{\log }, \bar{Y}_{2}^{\log }$. Finally, the composite of the equivalences of categories in the above display induces, by applying Theorem 4.2, an isomorphism of schemes

$$
Y_{1} \stackrel{\sim}{\rightarrow} Y_{2}
$$

that is functorial [in the evident sense!] with respect to $\bar{Y}_{1}^{\log }, \bar{Y}_{2}^{\log }$.

Proof. First, we consider assertion (i). The preservation of (i-a), (i-b), (i-c), (i-d), (i-e), (i-f), and (i-g) follows from an entirely similar argument to the argument applied in the proof of the preservation of the corresponding properties in Theorem 2.6, (i). Here, we observe that one verifies immediately, by arguing as in [5], Proposition 4.2, that

the minimal objects of $\overline{\mathscr{C}}^{\log }\left(\bar{X}_{i}^{\log }\right)$ are precisely the purely nonarchimedean objects of $\overline{\mathscr{C}}^{\log }\left(\bar{X}_{i}^{\log }\right)$ that arise from the minimal objects of $\mathscr{C}^{\log }\left(X_{i}^{\log }\right)$, where we write $X_{i}^{\log }$ for the underlying log scheme of $\bar{X}_{i}^{\log }$.

The preservation of (i-f ${ }^{\text {non }}$ ) now follows, in light of the preservation of (i-f), from an entirely similar argument - i.e., by considering epimorphisms as in [5], Proposition 4.5-to the argument applied to verify the category-theoretic characterization of purely nonarchimedean one-pointed objects given in [5], Corollary 4.1, (i). In light of the preservation of $\left(\mathrm{i}-\mathrm{f}^{\text {non }}\right)$, the preservation of (i-h) follows from an entirely similar argument to the argument applied in the proof of the preservation of (i-h) in Theorem 2.6, (i). In light of the preservation of (i-h), the preservation of $(\mathrm{i}-\mathrm{i})$ follows from an entirely similar argument to the argument applied in the proof of the preservation of (i-i) in Theorem 2.6, (i). The preservation of $\left(\mathrm{i}-\mathrm{i}^{\text {non }}\right)$ now follows from the preservation of (i-f), (i-f $\left.{ }^{\text {non }}\right),(\mathrm{i}-\mathrm{i})$, since [one verifies immediately that] the purely nonarchimedean submonic objects $\bar{Y}^{\log }$ of $\overline{\mathscr{C}}^{\log }\left(\bar{X}_{i}^{\log }\right)$ may be characterized as the submonically nonarchimedean submonic objects $\bar{Y}^{\log }$ of $\overline{\mathscr{C}}^{\log }\left(\bar{X}_{i}^{\log }\right)$. In light of the preservation of (i-i), the preservation of $(\mathrm{i}-\mathrm{j}),(\mathrm{i}-\mathrm{k})$ follows from an entirely similar argument to the argument applied in the proof of the preservation of $(\mathrm{i}-\mathrm{j})$, (i-k) in Theorem 2.6, (i). This completes the proof of assertion (i), except for the verification of the preservation of $(\mathrm{i}-1)$. 
Next, we consider assertion (ii). Suppose that $\bar{Y}_{i}^{\log }$ is submonic for $i=1,2$. Let $\bar{Z}_{i}^{\log } \rightarrow \bar{Y}_{i}^{\log }$ be a purely archimedean morphism of $\overline{\mathscr{C}}^{\log }\left(\bar{X}_{i}^{\log }\right)$ such that $\bar{Z}_{i}^{\log }$ is purely nonarchimedean submonic. Here, one verifies immediately that such a morphism $\bar{Z}_{i}^{\log } \rightarrow \bar{Y}_{i}^{\log }$ exists, and, moreover, that $\bar{Z}_{i}^{\log }$ may be characterized up to isomorphism as an object over $\bar{Y}_{i}^{\log }$ by the property that any arrow $\bar{T}^{\log } \rightarrow \bar{Y}_{i}^{\log }$ in $\overline{\mathscr{C}}^{\log }\left(\bar{X}_{i}^{\log }\right)$ such that $\bar{T}^{\log }$ is purely nonarchimedean submonic admits a unique factorization $\bar{T}^{\log } \rightarrow \bar{Z}_{i}^{\log } \rightarrow \bar{Y}_{i}^{\log }$. Thus, it follows from the portion of assertion (i) concerning the preservation of $\left(\mathrm{i}-\mathrm{i}^{\text {non }}\right)$ that we may assume without loss of generality that $\Phi\left(\bar{Z}_{1}^{\log }\right)=\bar{Z}_{2}^{\log }$. Moreover, since $\bar{Z}_{i}^{\log }$ is purely nonarchimedean, one verifies immediately from the various definitions involved that the full subcategory

$$
\overline{\mathscr{C}}^{\log }\left(\bar{Z}_{i}^{\log }\right) \subseteq \overline{\mathscr{C}}^{\log }\left(\bar{Y}_{i}^{\log }\right)
$$

admits a natural equivalence of categories $\mathscr{C}^{\log }\left(Y_{i}^{\log }\right) \stackrel{\sim}{\rightarrow} \overline{\mathscr{C}}^{\log }\left(\bar{Z}_{i}^{\log }\right)$ [cf. the statement of [5], Corollary 4.3]. Thus, by applying the portion of assertion (i) concerning the preservation of (i-k), one verifies immediately that assertion (ii) follows immediately follows from an entirely similar argument to the argument applied to verify Theorem 2.6, (ii). Finally, the portion of assertion (i) concerning the preservation of (i-1) follows from an entirely similar argument to the argument applied in the proof of the preservation of (i-1) in Theorem 2.6, (i). $\mathrm{SCH}^{\log }$

Next, we consider the analogue of Corollary 2.12 and Theorems 3.6, 3.8 for

THEOREM 4.6 (Reconstruction of the log scheme structure of arbitrary objects for $\left.\mathrm{SCH}^{\log }\right)$. For $i=1,2$, let $X_{i}^{\log }$ be a locally noetherian fs log scheme [cf. the discussion entitled "Log schemes" in §0]. We shall apply the notation introduced at the beginning of the present $\S 4$. Let

$$
\Phi: \mathrm{SCH}^{\log }\left(X_{1}^{\log }\right) \stackrel{\sim}{\rightarrow} \mathrm{SCH}^{\log }\left(X_{2}^{\log }\right)
$$

be an [arbitrary!] equivalence of categories. Then:

(i) $\Phi$ preserves the following:

(i-a) log-Dedekind objects;

(i-b) the set $\mathrm{SmCp}(-)$ associated to a log-Dedekind object;

(i-c) the subsets of the set $\operatorname{SmCp}(-)$ of $(\mathrm{i}-\mathrm{b})$ which are [N-]chains;

(i-d) partitions at elements of the set $\mathrm{SmCp}(-)$ of $(\mathrm{i}-\mathrm{b})$;

(i-e) orientable objects;

(i-f) homogeneous objects;

(i-g) one-pointed objects;

(i-h) point-hulls with one-pointed codomains;

(i-i) minimal point-hulls with one-pointed codomains;

(i-j) log-nodal objects. 
(ii) $\Phi$ preserves the following:

(ii-a) point-equivalent pairs of arrows;

(ii-b) the set-valued functor $\operatorname{LCPt}(-)$ [up to natural equivalence];

(ii-c) arrows which are minimal point-hulls;

(ii-d) scheme-like morphisms between arbitrary objects.

(iii) For $i=1,2$, let $Y_{i}^{\log }$ be an object of $\mathrm{SCH}^{\log }\left(X_{i}^{\log }\right)$; write $Y_{i}$ for the underlying scheme of $Y_{i}^{\log }$. Suppose further that $\Phi\left(Y_{1}^{\log }\right)=Y_{2}^{\log }$. Then $\Phi$ induces an equivalence of categories

$$
\left.\left.\left(\mathrm{SCH}\left(Y_{1}\right) \stackrel{\sim}{\rightarrow}\right) \mathrm{SCH}^{\log }\left(Y_{1}^{\log }\right)\right|_{\text {sch-lk }} \stackrel{\sim}{\rightarrow} \mathrm{SCH}^{\log }\left(Y_{2}^{\log }\right)\right|_{\text {sch-lk }}\left(\stackrel{\sim}{\rightarrow} \mathrm{SCH}\left(Y_{2}\right)\right)
$$

- where the equivalences in parentheses are the evident analogues for $\mathrm{SCH}, \mathrm{SCH}^{\log }$ of the natural equivalences of Definition 1.1, (iv) - that is functorial [in the evident sense!] with respect to $Y_{1}^{\log }, Y_{2}^{\log }$. Finally, the composite of the equivalences of categories in the above display induces, by applying Theorem 4.2, an isomorphism of schemes

$$
Y_{1} \stackrel{\sim}{\rightarrow} Y_{2}
$$

that is functorial [in the evident sense!] with respect to $Y_{1}^{\log }, Y_{2}^{\log }$.

(iv) There exists a unique isomorphism of log schemes

$$
X_{1}^{\log } \stackrel{\sim}{\rightarrow} X_{2}^{\log }
$$

such that $\Phi$ is isomorphic to the equivalence of categories induced by this isomorphism of log schemes $X_{1}^{\log } \stackrel{\sim}{\rightarrow} X_{2}^{\log }$.

Proof. In light of Theorem 4.4, the proof of assertion (i) (respectively, assertion (ii)) is entirely similar to the proof of Theorem 3.6, (i) (respectively, Corollary 2.12, (i)). Now assertion (iii) follows from the portion of assertion (ii) concerning the preservation of (ii-d) by applying an entirely similar argument to the argument applied to verify Corollary 2.12, (ii). Finally, it follows immediately from assertion (iii) that $\Phi$ preserves objects whose underlying scheme is noetherian [i.e., quasi-compact], and hence that $\Phi$ induces an equivalence of categories

$$
\operatorname{Sch}^{\log }\left(X_{1}^{\log }\right) \stackrel{\sim}{\rightarrow} \operatorname{Sch}^{\log }\left(X_{2}^{\log }\right)
$$

[i.e., as in Theorem 3.8]. Thus, assertion (iv) follows immediately from Theorem 3.8, (iii).

Finally, we consider analogues of Theorems 3.6, 3.8 for $\overline{\mathrm{Sch}}^{\log }, \overline{\mathrm{SCH}}^{\log }$. In order to formulate and prove these analogues, it will be necessary to introduce some new terminology [patterned after the terminology introduced in Definition 3.3], as follows.

Definition 4.7. Let $\overline{\mathscr{C}}^{\log } \in\left\{\overline{\mathrm{Sch}}^{\log }, \overline{\mathrm{SCH}}^{\log }\right\}$. If $\overline{\mathscr{C}}^{\log }=\overline{\mathrm{Sch}}^{\log }$, then set $\mathscr{C}^{\log } \stackrel{\text { def }}{=} \mathrm{Sch}^{\log }$; if $\overline{\mathscr{C}}^{\log }=\overline{\mathrm{SCH}}^{\log }$, then set $\mathscr{C}^{\log } \stackrel{\text { def }}{=} \mathrm{SCH}^{\log }$. Let $\bar{X}^{\log }$ be an 
arithmetic log scheme. We shall apply the notation introduced at the beginning of the present $\S 4$. Let $\bar{Y}^{\log }$ be a connected, non-submonic, $\mathscr{C}^{\log }$-log-Dedekind, submonically nonarchimedean [cf. Remark 4.7.1 below] object of $\overline{\mathscr{C}}^{\log }\left(\bar{X}^{\log }\right)$; write $Y^{\log }$ for the underlying $\log$ scheme of $\bar{Y}^{\log }$. Let $\gamma \in \mathscr{C}^{\log _{-}} \operatorname{SmCp}\left(\bar{Y}^{\log }\right) \stackrel{\text { def }}{=}$ $\operatorname{SmCp}\left(Y^{\log }\right)$. Write

$$
\operatorname{Mono}\left(\bar{Y}^{\log }\right)
$$

for the full subcategory of $\overline{\mathscr{C}}^{\log }\left(\bar{Y}^{\log }\right)$ determined by the arrows $\bar{H}^{\log } \rightarrow \bar{Y}^{\log }$ of $\overline{\mathscr{C}}^{\log }\left(\bar{X}^{\log }\right)$ which are monomorphisms in $\overline{\mathscr{C}}^{\log }\left(\bar{X}^{\log }\right)$.

(i) Let $C_{1}, C_{2} \subseteq \mathscr{C}^{\log _{-}} \operatorname{SmCp}\left(\bar{Y}^{\log }\right)$ be $\mathscr{C}^{\log }$-chains. Then we shall say that the pair of $\mathscr{C}^{\log }$-chains $\left\{C_{1}, C_{2}\right\}$ forms a $\ddot{\mathscr{C}}^{\log }$-partition at $\gamma$ if the $\mathscr{C}^{\log }$-chains $C_{1}$, $C_{2}$ satisfy the following conditions:

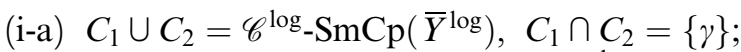

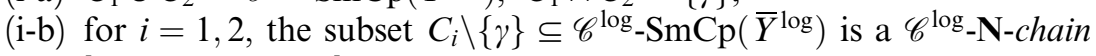
[hence nonempty];

(i-c) the $\mathscr{C}^{\log }-\mathbf{N}$-chains of (i-b) are "maximal" in the sense that every $\mathscr{C}^{\log }$-N-chain $C \subseteq \mathscr{C}^{\log }$-SmCp $\left(Y^{\log }\right)$ such that $\gamma \notin C$ is contained in $C_{i}$ for some $i \in\{1,2\}$;

(i-d) if, for $i=1,2$, we write $\Psi_{i}$ for the subfunctor of the contravariant functor determined by the terminal object [i.e., $\bar{Y}^{\log }$ ] of $\operatorname{Mono}\left(\bar{Y}^{\log }\right)$ that consists of objects $\bar{h}^{\log }: \bar{H}^{\log } \hookrightarrow \bar{Y}^{\log }$ of $\operatorname{Mono}\left(\bar{Y}^{\log }\right)$ such that every composite morphism $\bar{H}_{*}^{\log } \hookrightarrow \bar{H}^{\log } \mapsto \bar{Y}^{\log }$, where $\bar{H}_{*}^{\log } \longmapsto \bar{H}^{\log }$ is a minimal point of $\bar{H}^{\log }$, determines an underlying morphism in $\mathscr{C}^{\log }\left(Y^{\log }\right)$ that factors through some representative of an element $\in C_{i}\left(\subseteq \mathscr{C}^{\log _{-}} \operatorname{SmCp}\left(\bar{Y}^{\log }\right)\right)$, then $\Psi_{i}$ is representable by an object $\bar{h}_{i}^{\log }: \bar{Y}_{i}^{\log } \mapsto \bar{Y}^{\log }$ of $\operatorname{Mono}\left(\bar{Y}^{\log }\right)$.

We shall say that $\bar{Y}^{\log }$ is $\ddot{\mathscr{C}}^{\log }$-orientable if $\bar{Y}^{\log }$ admits a $\ddot{\mathscr{C}}^{\log }$-partition at every element of $\mathscr{C}^{\log }-\operatorname{SmCp}\left(\bar{Y}^{\log }\right)$.

(ii) Let $\left\{C_{1}, C_{2}\right\}$ be a $\ddot{\mathscr{C}}^{\log }$-partition at $\gamma$. Suppose that $\bar{h}_{1}^{\log }, \bar{h}_{2}^{\log }$ are as in (i-d). Then we shall say that the $\ddot{\mathscr{C}}^{\log }$-partition $\left\{C_{1}, C_{2}\right\}$ is $\ddot{\mathscr{C}}^{\log }$-seamless if the following condition is satisfied:

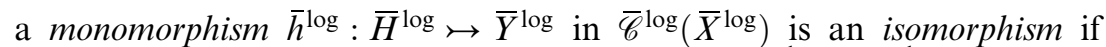
and only if, for $i=1,2$, the projection $\bar{H}^{\log } \times_{\bar{Y}_{\log }} \bar{Y}_{i}^{\log } \rightarrow \bar{Y}_{i}^{\log }$ associated to the fiber product determined by $\bar{h}^{\log }$ and $\bar{h}_{i}^{\log }$ is an isomorphism.

We shall say that $\bar{Y}^{\log }$ is $\ddot{\mathscr{C}}^{\log }$-homogeneous if $\bar{Y}^{\log }$ is $\ddot{\mathscr{C}}^{\log }$-orientable, and,

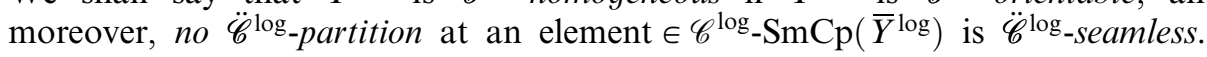

Remark 4.7.1. Let $\bar{Y}^{\log }$ be as in Definition 4.7, i.e., a connected, nonsubmonic, $\mathscr{C}^{\log }$-log-Dedekind, submonically nonarchimedean object of $\overline{\mathscr{C}}^{\log }\left(\bar{X}^{\log }\right)$. Write $Y^{\log }$ for the underlying $\log$ scheme of $\bar{Y}^{\log } ; \bar{Y}$ for the underlying arithmetic 
scheme of $\bar{Y}^{\log } ; Y$ for the underlying scheme of $\bar{Y} ; K^{\log }$ for the compact set that determines the archimedean structure of $\bar{Y} \log$ [i.e., the set " $H$ " of [5], Definition 4.2, (ii)]; $K$ for the compact set that determines the archimedean structure of $\bar{Y}$ [i.e., the set " $H$ " of [5], Definition 4.2, (i)]. Thus, it follows immediately from the various definitions involved that we have a natural surjection $K^{\log } \rightarrow K$ whose fibers are compact [cf. the discussion of such compact subsets in the proof of [5], Lemma 4.1]. Now observe that the assumption that $\bar{Y}^{\log }$ is submonically nonarchimedean implies [cf. Proposition 3.2, (i), (ii)] that

$K$ is a finite compact set which is supported over the nodal points of $Y^{\log }$.

Since the finiteness of $K$ implies that any [e.g., open!] subset of $K$ is compact [i.e., relative to the topology induced by $K$ ], we thus conclude that

any subset of $K^{\log }$ that arises as the inverse image via the natural surjection $K^{\log } \rightarrow K$ of a subset of $K$ is compact [i.e., relative to the topology induced by $\left.K^{\log }\right]$.

In particular, it follows that any open subscheme $Z \subseteq Y$ determines, in a natural

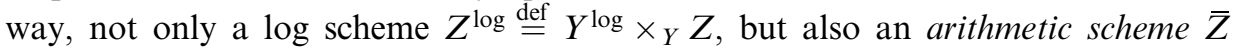
and an arithmetic log scheme $\bar{Z}^{\log }$ [i.e., by considering the subsets of $K, K^{\log }$ consisting of points that map to points of $Z(\subseteq Y)]$. Moreover, one verifies immediately that

$\bar{Z}^{\log }$ (respectively, $\bar{Z}$ ) represents the covariant subfunctor of the functor represented by $\bar{Y}^{\log }$ (respectively, $\bar{Y}$ ) on the category of arithmetic log schemes (respectively, arithmetic schemes) determined by the condition on a morphism to $\bar{Y} \log$ (respectively, $\bar{Y}$ ) that the associated underlying morphism of schemes maps into $Z \subseteq Y$.

These observations may be applied, for instance, to open subschemes of $Y$ that arise as images of open immersions of the sort discussed in Proposition 3.4, (ii).

THEOREM 4.8 (Reconstruction of the arithmetic log scheme structure of arbitrary objects for $\left.\overline{\mathrm{Sch}}^{\log }, \overline{\mathrm{SCH}}^{\log }\right)$. Let $\overline{\mathscr{C}}^{\log } \in\left\{\overline{\mathrm{Sch}}^{\log }, \overline{\mathrm{SCH}}^{\log }\right\}$. If $\overline{\mathscr{C}}^{\log }=$ $\overline{\mathrm{Sch}}^{\log }$, then set $\mathscr{C}^{\log } \stackrel{\text { def }}{=} \mathrm{Sch}^{\log }$; if $\overline{\mathscr{C}}^{\log }=\overline{\mathrm{SCH}}^{\log }$, then set $\mathscr{C}^{\log } \stackrel{\text { def }}{=} \mathrm{SCH}^{\log }$. For $i=1,2$, let $\bar{X}_{i}^{\log }$ be an arithmetic log scheme [cf. [5], Definition 4.2, (ii)]. We shall apply the notation introduced at the beginning of the present $\$ 4$. Let

$$
\Phi: \overline{\mathscr{C}}^{\log }\left(\bar{X}_{1}^{\log }\right) \stackrel{\sim}{\rightarrow} \overline{\mathscr{C}}^{\log }\left(\bar{X}_{2}^{\log }\right)
$$

be an [arbitrary!] equivalence of categories. Then:

(i) Let $\bar{Y}^{\log }, \bar{Z}^{\log }$ be objects of $\overline{\mathscr{C}}^{\log }\left(\bar{X}_{i}^{\log }\right)$, for some $i \in\{1,2\}$, that are connected, non-submonic, $\mathscr{C}^{\log }$-log-Dedekind, and submonically nonarchime-dean; write $Y^{\log }, Z^{\log }$ for the underlying $\log$ schemes of $\bar{Y}^{\log }, \bar{Z}^{\log }$, respectively. 
Suppose further that $\bar{Z}^{\log }$ is purely nonarchimedean. Then the following properties hold:

(i-a $\left.{ }_{Y}\right)$ every $\ddot{\mathscr{C}}^{\log }$-partition at an element $\gamma \in \mathscr{C}^{\log }-\operatorname{SmCp}\left(\bar{Y}^{\log }\right)$ determines $a \mathscr{C}^{\log }$-partition at $\gamma$;

$\left(\mathrm{i}-\mathrm{a}_{Z}\right)$ there $i s$ a natural bijective correspondence between $\ddot{\mathscr{C}}^{\log }$.

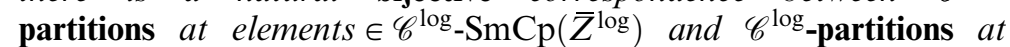
elements $\in \mathscr{C}^{\log }-\operatorname{SmCp}\left(\bar{Z}^{\log }\right)$;

$\left(\mathrm{i}-\mathrm{b}_{Y}\right)$ if $\bar{Y}^{\log }$ is $\ddot{\mathscr{C}}^{\log }$-orientable, then $\bar{Y}^{\log }$ is $\mathscr{C}^{\log }$-orientable;

$\left(\mathrm{i}-\mathrm{b}_{Z}\right) \bar{Z}^{\log }$ is $\ddot{\mathscr{C}}^{\log }$-orientable if and only if $\bar{Z}^{\log }$ is $\mathscr{C}^{\log }$-orientable;

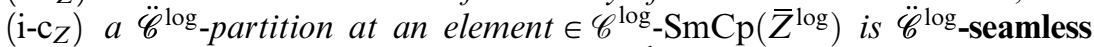

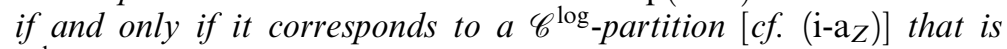
$\mathscr{C}^{\log }$-seamless;

$\left(\mathrm{i}-\mathrm{d}_{Y}\right)$ if $\bar{Y}^{\log }$ is $\ddot{\mathscr{C}}^{\log -h o m o g e n e o u s,}$ then it is one-pointed, and $Y_{\mathrm{sm}}^{\log }$ is empty;

$\left(\mathrm{i}-\mathrm{d}_{Z}\right) \bar{Z}^{\log }$ is $\ddot{\mathscr{C}}^{\log -h o m o g e n e o u s}$ if and only if $\bar{Z}^{\log }$ is $\mathscr{C}^{\log }$-homogeneous.

(ii) $\Phi$ preserves the following:

(ii-a) $\mathscr{C}^{\log }$-log-Dedekind objects;

(ii-b) the set $\mathscr{C}^{\log _{-} \mathrm{SmCp}(-)}$ associated to a $\mathscr{C}^{\log }$-log-Dedekind object;

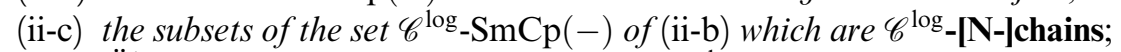

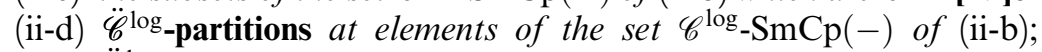

(ii-e) $\ddot{\mathscr{C}}^{\log }$-orientable objects;

(ii-f) $\ddot{\mathscr{C}}^{\log }$-homogeneous objects;

(ii-g) one-pointed objects;

(ii-h) point-hulls with one-pointed codomains;

(ii-i) minimal point-hulls with one-pointed codomains.

(iii) For $i=1,2$, let $\bar{Y}_{i}^{\log }$ be an object of $\overline{\mathscr{C}}^{\log }\left(\bar{X}_{i}^{\log }\right)$; write $Y_{i}^{\log }$ for the underlying log scheme of $\bar{Y}_{i}^{\log }$. Suppose further that $\Phi\left(\bar{Y}_{1}^{\log }\right)=\bar{Y}_{2}^{\log }$. Then $\bar{Y}_{1}^{\log }$ is purely nonarchimedean if and only if $\bar{Y}_{2}^{\log }$ is. In particular, $\Phi$ induces an equivalence of categories

$$
\mathscr{C}^{\log }\left(Y_{1}^{\log }\right) \stackrel{\sim}{\rightarrow} \mathscr{C}^{\log }\left(Y_{2}^{\log }\right)
$$

that is functorial [in the evident sense!] with respect to $\bar{Y}_{1}^{\log }, \bar{Y}_{2}^{\log }$. Finally, the equivalence of categories in the above display induces, by applying Theorems 3.8, (iii); 4.6, (iv), an isomorphism of $\log$ schemes

$$
Y_{1}^{\log } \stackrel{\sim}{\rightarrow} Y_{2}^{\log }
$$

that is functorial [in the evident sense!] with respect to $\bar{Y}_{1}^{\log }, \bar{Y}_{2}^{\log }$.

(iv) There exists a unique isomorphism of arithmetic log schemes

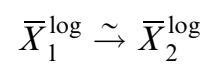

such that $\Phi$ is isomorphic to the equivalence of categories induced by this isomorphism of arithmetic log schemes $\bar{X}_{1}^{\log } \stackrel{\sim}{\rightarrow} \bar{X}_{2}^{\log }$. 
Proof. First, we consider assertion (i). Properties $\left(\mathrm{i}-\mathrm{a}_{Y}\right), \quad\left(\mathrm{i}-\mathrm{a}_{Z}\right),\left(\mathrm{i}-\mathrm{b}_{Y}\right)$, $\left(\mathrm{i}-\mathrm{b}_{Z}\right),\left(\mathrm{i}-\mathrm{c}_{Z}\right)$, and $\left(\mathrm{i}-\mathrm{d}_{Z}\right)$ follow formally from the definitions [cf. also the first display in the proof of Theorem 4.5]. Property $\left(\mathrm{i}-\mathrm{d}_{Y}\right)$ then follows, in light of properties $\left(\mathrm{i}-\mathrm{a}_{Y}\right)$ and $\left(\mathrm{i}-\mathrm{b}_{Y}\right)$ [cf. also the first display in the proof of Theorem 4.5], by applying a similar argument to the argument [i.e., involving Proposition 3.4, (ii)] applied in the proof of Proposition 3.4, (iii). Here, we note that one must apply the assumption [cf. the beginning of Definition 4.7] that any $\ddot{\mathscr{C}}^{\log }$. homogeneous object is submonically nonarchimedean in order to conclude that

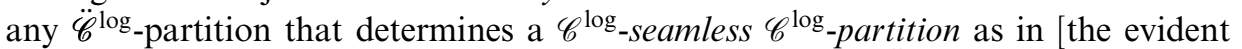

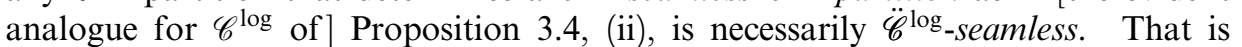
to say, this assumption that any $\ddot{\mathscr{C}}^{\log }$-homogeneous object is submonically nonarchimedean implies [cf. the discussion of Remark 4.7.1] that the discrepancy

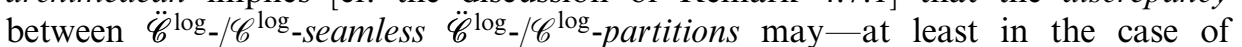
$\mathscr{C}^{\log }$-seamless $\mathscr{C}^{\log }$-partitions as in [the evident analogue for $\mathscr{C}^{\log }$ of] Proposition 3.4, (ii)-be ignored. This completes the proof of assertion (i).

Next, we observe that, in light of Theorem 4.5, (i), (ii), assertion (ii) follows by applying a similar argument to the argument applied to verify Theorem 3.6, (i). Here, we observe that the preservation of the crucial property of being submonically nonarchimedean [cf. the beginning of Definition 4.7] follows formally from the portion of Theorem 4.5, (i), concerning the preservation of (i-f), (i-f $\left.{ }^{\text {non }}\right)$. Also, we observe, with regard to the preservation of (ii-g), that, by applying

- the property $\left(\mathrm{i}-\mathrm{d}_{Y}\right)$ of assertion (i) in place of Proposition 3.4, (iii), and

- the property $\left(i-d_{Z}\right)$ of assertion (i), together with the evident analogue for $\mathscr{C}^{\log }$ of Proposition 3.4, (iv), in place of Proposition 3.4, (iv),

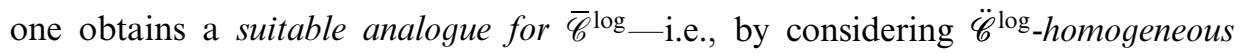
objects - of the characterization of one-pointed objects given in Proposition 3.5, (i). This completes the proof of assertion (ii).

Next, we consider assertion (iii). First, let us observe that the portion of assertion (ii) concerning the preservation of (ii-g), (ii-i) allows one to circumvent the application of [5], Propositions 4.3, 4.4, in the theory of [5], §4. One thus obtains-i.e., by considering epimorphisms as in [5], Proposition 4.5category-theoretic characterizations of the purely nonarchimedean one-pointed objects of $\overline{\mathscr{C}}^{\log }\left(\bar{X}_{i}^{\log }\right)$ as in [5], Corollary 4.1, (i), and of the purely archimedean morphisms of $\overline{\mathscr{C}}^{\log }\left(\bar{X}_{i}^{\log }\right)$ as in [5], Corollary 4.1, (ii) [cf. also Proposition 1.4, (iii), (v), of the present paper]. In particular, we obtain a category-theoretic characterization, as in [5], Corollary 4.2, of the purely nonarchimedean objects of $\overline{\mathscr{C}}^{\log }\left(\bar{X}_{i}^{\log }\right)$ and hence, by applying Theorems 3.8 , (iii); 4.6, (iv), a categorytheoretic reconstruction of the underlying log scheme of an object of $\overline{\mathscr{C}}^{\log }\left(\bar{X}_{i}^{\log }\right)$, as in [5], Corollary 4.3. This completes the proof of assertion (iii). Finally, assertion (iv) follows from assertion (iii) [cf. the proof of [5], Theorem 5.1], by applying the "logarithmic global compatibility" established in [5], Lemma 5.2. 


\section{Appendix}

In the present Appendix, we discuss in more detail, at the level of individual propositions, lemmas, corollaries, theorems, and examples, the validity of the theory developed in [4] and [5].

First, we recall that the errors discussed in the Introduction of the present paper have no effect on [4], §1. The effect of these errors on the validity of the statements [second column], as well as on the validity of the proofs [third column], of the individual propositions, lemmas, corollaries, and theorems of [4], §2, is summarized in Fig. 1 below. Here, the symbol " $\bigcirc$ " indicates no effect on the validity in question; the symbol " $x$ " indicates some effect on the validity in question. Certain results that concern equivalences are divided into sufficiency and necessity portions. Moreover, the necessity portion of [4], Proposition 2.3, is divided into

- a portion concerning whether or not the underlying morphism of schemes is a monomorphism in the case where the given morphism of $\log$ schemes is scheme-like,

- a portion concerning whether or not the induced morphism of groupifications of characteristics is surjective, and

- a portion concerning whether or not the underlying morphism of schemes is a monomorphism in the case where the given morphism of $\log$ schemes is not scheme-like.

\begin{tabular}{|c|c|c|c|}
\hline $\begin{array}{l}\text { Individual propositions/lemmas/ } \\
\text { corollaries/theorems }\end{array}$ & $\begin{array}{l}\text { Validity of } \\
\text { statement }\end{array}$ & $\begin{array}{l}\text { Validity } \\
\text { of proof }\end{array}$ & $\begin{array}{l}\text { Explicit logical } \\
\text { application in } \\
\text { the present paper }\end{array}$ \\
\hline $\begin{array}{l}2.3 \text { (surjectivity portion of necessity); } 2.5 ; 2.6, \\
\text { (i), (iii); 2.6, (ii) (closed immersion, [final] } \\
\text { surjectivity portions); } 2.16 \text { [cf. Remark 3.7.1] }\end{array}$ & $\bigcirc$ & $\bigcirc$ & $\bigcirc$ \\
\hline $\begin{array}{l}2.1 ; 2.2 ; 2.3 \text { (sufficiency); } 2.3 \text { (monomorphism } \\
\text { portion of necessity: scheme-like case); } 2.7 \text {, } \\
\text { (i), (ii); } 2.8 ; 2.12 \text {, (i) (necessity); 2.12, (ii); } \\
\text { 2.17;2.18;2.19, (i);2.20 }\end{array}$ & $\bigcirc$ & O & $\times$ \\
\hline 2.4 & $\bigcirc$ & $\times$ & $\triangle$ \\
\hline $2.14 ; 2.15 ; 2.19$, (ii); 2.13 & O & $x$ & $x$ \\
\hline $\begin{array}{l}2.3 \text { (monomorphism portion of necessity: non- } \\
\text { scheme-like case); 2.6, (ii) (isomorphism portion); } \\
\text { 2.7, (iii);2.9;2.10;2.12, (i) (sufficiency) }\end{array}$ & $x$ & $\times$ & $x$ \\
\hline
\end{tabular}

FIGURE 1. Validity of individual propositions/lemmas/corollaries/theorems of [4] 
The statement of [4], Lemma 2.6, (ii), is also divided into a closed immersion portion, an isomorphism portion, and a [final] surjectivity portion. We also indicate, in the fourth column of Fig. 1, whether [" $\bigcirc$ "] or not [" $x$ "] the result in question is applied, in an explicit [i.e., via a direct reference] logical sense, in the present paper. The data of this fourth column does not include references for the statements of definitions/conditions or references made for the sake of pointing out content that is related in an expository sense [i.e., but not in a logical sense!]. The unique " $\triangle$ " in this fourth column in the case of [4], Proposition 2.4, indicates that although we apply this result in an explicit logical sense in the present paper [i.e., despite the fact that the proof given in [4] is in error!], this does not result in any logical gaps, since the proof of [4], Proposition 2.4, given in [4] may be repaired if, instead of applying [4], Proposition 2.3, one applies Proposition 1.4, (vi), of the present paper [i.e., which corresponds to the necessity portion of [4], Proposition 2.3, in the case of submonic log schemes].

Next, we consider the effect of the errors discussed in the Introduction of the present paper on [5]. Here, we recall that [5], §1, consists of an expository introduction to the theory of [5], while [5], §2, is devoted to a discussion of the notations and conventions applied in [5]. Thus, it suffices to consider the effect of the errors discussed in the Introduction of the present paper on [5], $3, \S 4$, $\S 5$. The effect of these errors on the validity of the statements [second column], as well as on the validity of the proofs [third column], of the individual propositions ["P"], lemmas ["L"], corollaries ["C" $C$ "], theorems ["T"], and examples ["E"] of [5], $33, \S 4, \S 5$, is summarized in Fig. 2 below. Here, the symbol " $\bigcirc$ " indicates no effect on the validity in question; the symbol " $x$ " indicates some effect on the validity in question; the numbers in parentheses indicate, for ease of reference, the corresponding result in the preprint version [available on the homepage http://www.kurims.kyoto-u.ac.jp/ motizuki/papers-english.html] of [5]. We also indicate, in the fourth column of Fig. 2, whether [" $\bigcirc$ "] or not [" $x$ "] the result in question is applied, in an explicit [i.e., via a direct reference] logical sense, in the present paper. The data of this fourth column does not include references for the statements of definitions/conditions or references made for the sake of pointing out content that is related in an expository sense [i.e., but not in a logical sense!]. The three " $\triangle$ ' $s$ " in this fourth column indicate the following state of affairs:

- The results marked with a " $\triangle$ " are indeed applied in an explicit logical sense in the present paper in the proofs of Theorems 4.3, 4.5, 4.8-i.e., despite the fact that the proofs given in [5] of these results are in error!

- The explicit logical application of these results in the present paper does not, however, result in any logical gaps for the following reason: In the case of the first and third (respectively, case of the second) " $\triangle$ ", the only problem with the proofs given in [5] is that they rely on the reconstruction of one-pointed objects given in [5], Proposition 4.3 [i.e., which is in error!] (respectively, on [5], Corollaries 4.2, 4.3 [i.e., 
whose proofs are in error!]). On the other hand, these results are only applied in the present paper in situations in which the onepointed objects have already been reconstructed (respectively, in which results corresponding to [5], Corollaries 4.2, 4.3, have already been proven).

- Put another way, one may think of the application in the present paper of the results marked with a " $\triangle$ " as consisting of a "similar argument" to the argument given in [5]-i.e., a "similar argument" which does not suffer from the logical gaps of [5], since, in the case of the first and third (respectively, case of the second) " $\triangle$ ", this "similar argument" is only applied in situations in which the one-pointed objects have already been reconstructed (respectively, in which results corresponding to [5], Corollaries 4.2, 4.3, have already been proven).

\begin{tabular}{|c|c|c|c|}
\hline $\begin{array}{l}\text { Individual propositions/lemmas/corollaries/ } \\
\text { theorems/examples }\end{array}$ & $\begin{array}{c}\text { Validity of } \\
\text { statement }\end{array}$ & $\begin{array}{c}\text { Validity } \\
\text { of proof }\end{array}$ & $\begin{array}{c}\text { Explicit logical } \\
\text { application in } \\
\text { the present paper }\end{array}$ \\
\hline L4.1 (2.5); P4.2 (2.6); C4.1 (2.10), (ii) & $\bigcirc$ & $\bigcirc$ & $\bigcirc$ \\
\hline P4.1 (2.4); P4.4 (2.8), (i), (ii); E5.1 (3.5) & $\bigcirc$ & $\bigcirc$ & $\times$ \\
\hline P4.4 (2.8), (iii); C4.2 (2.11) & $\bigcirc$ & $\times$ & $\triangle$ \\
\hline L5.1 (3.2); L5.2 (3.3) & $\bigcirc$ & $\times$ & $\times$ \\
\hline T3.1 (1.1); T5.1 (3.4); C4.3 (2.12) & $\bigcirc$ & $\times$ & $\triangle$ \\
\hline P4.5 (2.9); C4.1 (2.10), (i) & $\times$ & $\times$ & $\times$ \\
\hline P4.3 (2.7) & & $\times$ & $\times$ \\
\hline
\end{tabular}

FIGURE 2. Validity of individual propositions/lemmas/corollaries/theorems/examples of [5]

\section{BIBLIOGRAPHY}

[1] K. Kato, Logarithmic structures of Fontaine-Illusie, Algebraic analysis, geometry, and number theory, Johns Hopkins Univ. Press, Baltimore, MD, 1989, 191-224.

[2] K. Kato, Toric singularities, Amer. J. Math. 116 (1994), 1073-1099.

[3] S. Mochizuki, Extending families of curves over log regular schemes, J. Reine Angew. Math. 511 (1999), 43-71.

[ 4 ] S. Mochizuki, Categorical representation of locally noetherian log schemes, Adv. Math. 188 (2004), 222-246.

[5] S. MochizuKi, Categories of $\log$ schemes with archimedean structures, J. Math. Kyoto Univ. 44 (2004), 891-909. 
[6] S. Mochizuki, The geometry of Frobenioids I: The general theory, Kyushu J. Math. 62 (2008), 293-400.

Shinichi Mochizuki

Research Institute for Mathematical Sciences

KYOTO UNIVERSITY

Куото 606-8502

JAPAN

E-mail: motizuki@kurims.kyoto-u.ac.jp 